\title{
Airflow Sensing with Arrays of Hydrogel Supported Artificial Hair Cells
}

\author{
Rodrigo Sarlo \\ Thesis submitted to the Faculty of the \\ Virginia Polytechnic Institute and State University \\ in partial fulfillment of the requirements for the degree of
}

Master of Science
in
Mechanical Engineering

Donald J. Leo, Chair

Pablo Tarazaga

Mary Kasarda

January 19, 2015

Blacksburg, Virginia

Keywords: artificial hair cell, spectral analysis, frequency decomposition, array sensing, lipid bilayer

Copyright 2015, Rodrigo Sarlo 


\title{
Airflow Sensing with Arrays of Hydrogel Supported Artificial Hair Cells
}

\author{
Rodrigo Sarlo \\ Virginia Polytechnic Institute and State University \\ Donald J. Leo, Advisor
}

\begin{abstract}
Arrays of fully hydrogel-supported, artificial hair cell (AHC) sensors based on bilayer membrane mechanotransduction are designed and characterized to determine sensitivity to multiple stimuli. The work draws upon key engineering design principles inspired by the characteristics of biological hair cells, primarily the use of slender hair-like structures as flow measurement elements. Many hair cell microelectromechanical (MEMS) devices to sense fluid flow have already been built based on this principle. However, recent developments in lipid bilayer applications, namely physically encapsulated bilayers and hydrogel interface bilayers, have facilitated the development of AHCs made primarily from biomolecular materials. The most current research in this field of "membrane based AHCs," shows promise, yet still lacks the modularity to create large sensor arrays similar to those in nature.

This paper presents a novel bilayer based AHC platform, developed for array implementation by applying some of the core design principles of biological hair cells. These principles are translated into key design, fabrication and material considerations toward improved sensor sensitivity and modularity. Single hair cell responses to base excitation and short air pulses are to investigate the dynamic coupling between hair and bilayer membrane transducer. In addition, a spectral analysis of the AHC system under varying voltages and air flow velocities helps to build simple, predictive models for the sensitivity properties of the AHC. And finally, based on these results, we implement a spatial sensing strategy that involves mapping frequency content to stimulus location by "tuning" linear, three-unit arrays of AHCs.
\end{abstract}

Individual AHC sensors characterization results demonstrate peak current outputs in the nanoamp range and measure flow velocities as high as $72 \mathrm{~m} / \mathrm{s}$. Characterization of the AHC response to base excitation and air pulses show that membrane current oscillates with the first three bending modes of the hair. Output magnitudes reflect of vibrations near the base of the hair. A 2 degree-of-freedom Rayleigh-Ritz approximation of the system dynamics yields estimates of $19 \mathrm{~N} / \mathrm{m}$ and $0.0011 \mathrm{Nm} / \mathrm{rad}$ for the equivalent linear and torsional stiffness of the hair's hydrogel base, although double modes suggest non-symmetry 
in the gel's linear stiffness. The sensor output scales linearly with applied voltage (1.79 $\mathrm{pA} / \mathrm{V}$ ), avoiding a higher-order dependence on electrowetting effects. The free vibration amplitude of the sensor also increases in a linear fashion with applied airflow pressure (3.39 $\mathrm{pA} / \mathrm{m} \mathrm{s}^{-1}$ ). Array sensing tests show that the bilayers' consistent spectral responses allow for an accurate localization of the airflow source. However, temporal variations in bilayer size affect sensitivity properties and make airflow magnitude estimation difficult. The overall successful implementation of the array sensing method validates the sensory capability of the bilayer based AHC. 


\section{Dedication}

To my parents, Guillermo and Maria, and to my two brothers, Alvaro and Thomas. 


\section{Acknowledgements}

Many people have graciously contributed their time in support of my work. Firstly, I would like to recognize the generous financial support of the National Science Foundation, Grant CMMI-1129951. Secondly, I would like to thank Dr. Donald Leo for his patience, advice and guidance throughout this project. Additional thanks go to Dr. Pablo Tarazaga, who donated useful insight into vibrations concepts as well some of his laboratory equipment. I also thank my friends and lab mates whose collaboration led to these results: Engin Sengezer, who graciously helped me develop the code to the Rayleigh-Ritz model; Ethan Robinson, whose contributions to the experimental setup were indispensable for the success of this work; and Joseph Najem, Eric Freeman and Kevin Crowley, whose thoughtful laboratory discussion contributed greatly to my understanding of lipid bilayers. A final piece of gratitude is reserved to the my parents and Leydi Zora, who supported and encouraged me during the writing of this paper.

Rodrigo SARLO 


\section{Contents}

Contents vi vi v v v v v

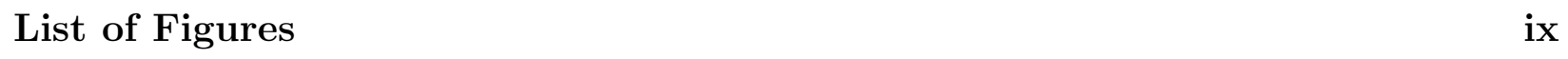

\begin{tabular}{ll}
\hline List of Tables & xvi
\end{tabular}

\begin{tabular}{llr}
\hline & Introduction and Literature Review & 1
\end{tabular}

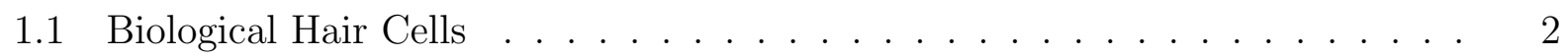

1.1 .1 Basic structure and mechanics . . . . . . . . . . . . . . . . . . 2

1.1 .2 Hair cell diversity in nature . . . . . . . . . . . . . . . . . . . . 4

1.2 The Lipid Bilayer . . . . . . . . . . . . . . . . . . . . . . . . . . . . . . . 10

1.2 .1 Structure and biological function . . . . . . . . . . . . . . . . 11

1.2 .2 Formation of Synthetic Bilayers . . . . . . . . . . . . . . . . . . . . . 12

$1.2 .3 \quad$ Bilayer Mechanoelectrical Transduction . . . . . . . . . . . . . . . . . 16

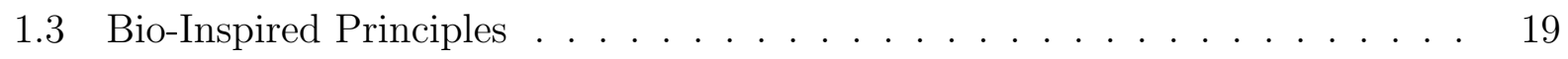

1.3 .1 Cilia as flow measurement elements . . . . . . . . . . . . . . . . . . . 20

1.3 .2 Array sensing . . . . . . . . . . . . . . . . . . . . 20

1.3 .3 Biomolecular Transduction . . . . . . . . . . . . . . . . . . . . . . . 21

1.4 Bio-inspired Artificial Hair Cells . . . . . . . . . . . . . . . . . . . . . . . . . 21

1.4 .1 MEMS based AHCS . . . . . . . . . . . . . . . . . . . . . . . . . 22

1.4 .2 Bilayer Based AHCs . . . . . . . . . . . . . . . . . . . . . 24

1.5 Document Overview $\ldots \ldots \ldots \ldots \ldots \ldots$ 


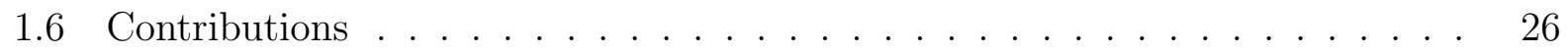

\begin{tabular}{|lll}
2 & Design and Testing Methods for a Novel Artificial Hair Cell Array & 27
\end{tabular}

2.1 The Artificial Hair Cell (AHC) $\ldots \ldots \ldots \ldots$

2.1 .1 Components of the AHC Bilayer Membrane . . . . . . . . . . . 27

2.1 .2 Unit Fabrication $\ldots \ldots \ldots \ldots \ldots$

2.1 .3 Array Assembly . . . . . . . . . . . . . . . . . . . . . . 30

2.1 .4 Design Evaluation . . . . . . . . . . . . . . . . . . . . . . . 31

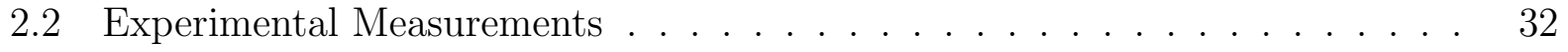

2.3 AHC Testing Methods . . . . . . . . . . . . . . . . . . . . . . . 35

2.3 .1 Base Excitation $\ldots \ldots \ldots \ldots$. . . . . . . . . . . . . . 36

$2.3 .2 \quad$ Air Pulse Excitation $\ldots \ldots \ldots \ldots$

2.3 .3 Constant Airflow Excitation . . . . . . . . . . . . . . . . 37

\begin{tabular}{lll}
\hline 3 & Theory and Modelling & 40
\end{tabular}

3.1 Model of the Hair-Hydrogel System . . . . . . . . . . . . . . . . . . . . 41

3.2 Bilayer Mechanoelectrical Transduction . . . . . . . . . . . . . . . . . . . 43

$3.2 .1 \quad$ Circuit Representation . . . . . . . . . . . . . . . . . . . . . 43

3.2 .2 Current Generation Through Vibration . . . . . . . . . . . . . . 44

3.2 .3 Electrowetting Effect $\ldots \ldots \ldots \ldots$

4 Characterization of AHC Sensor's Dynamic Response 48

$4.1 \quad$ Control Testing . . . . . . . . . . . . . . . . . . . . . . . . . . . . . . . . . . . . . . . . . . . 49

4.1 .1 Sources of noise $\ldots \ldots \ldots \ldots$

$4.1 .2 \quad$ Bilayer versus no bilayer $\ldots \ldots \ldots \ldots \ldots \ldots$

4.1 .3 Vibration isolation between $\mathrm{AHC}$ units $\ldots \ldots \ldots \ldots$

4.2 Base Excitation Results $\ldots \ldots \ldots \ldots$

$4.3 \quad$ Air Pulse Excitation Results $\ldots \ldots \ldots \ldots$. . . . . . . . . . . . . 57

$\begin{array}{lll}5 & \text { AHC Sensitivity Properties } & 60\end{array}$ 
5.1 Sensitivity to Applied Voltage $\ldots \ldots \ldots$. . . . . . . . . . . . . 6

$5.1 .1 \quad$ Electrowetting Effect and Voltage Capacitance Relationship . . . . 60

5.1 .2 Voltage to current output relationship $\ldots \ldots \ldots \ldots$. . . . . . 62

5.2 Sensitivity to Airflow . . . . . . . . . . . . . . . . . . . . . 64

5.2 .1 Current output versus airflow magnitude $\ldots \ldots \ldots \ldots$

\begin{tabular}{|lll}
6 & Flow Localization using Arrays of AHCs & 67
\end{tabular}

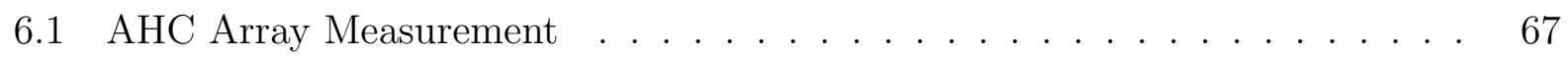

$6.2 \quad$ Hair Frequency Response Decomposition $\ldots \ldots \ldots$

$6.2 .1 \quad$ Air Velocity Profile $\ldots \ldots \ldots \ldots$

$6.2 .2 \quad$ Reference Signals and Sensitivity Analysis . . . . . . . . . . 76

$6.2 .3 \quad$ Flow Position \& Magnitude Mapping via HFRD . . . . . . . . . . . . 77

\begin{tabular}{lll}
\hline 7 & Summary and Conclusions & 84
\end{tabular}

7.1 Research Summary . . . . . . . . . . . . . . . . . . . . . . . . . . . 84

7.2 Conclusions $\ldots \ldots \ldots \ldots \ldots \ldots$

$7.2 .1 \quad$ AHC Design and Characterization . . . . . . . . . . . . 86

$7.2 .2 \quad$ AHC Sensitivity Properties $\ldots \ldots \ldots \ldots \ldots$. . . . . . . . 87

$7.2 .3 \quad$ Array Implementation $\ldots \ldots \ldots$. . . . . . . . . . . . . . . . 88

7.3 Contributions $\ldots \ldots \ldots \ldots \ldots \ldots \ldots$

\begin{tabular}{lr}
\hline Bibliography & 90
\end{tabular}

\begin{tabular}{lr}
\hline A Substrate Dimensions & 98
\end{tabular}

\begin{tabular}{ll}
\hline B Rayleigh-Ritz Calculation Algorithm & 100
\end{tabular}

\begin{tabular}{|ll|}
\hline C Hair Frequency Response Decomposition Top-Level Algorithm & 102
\end{tabular} 


\section{List of Figures}

1.1 Diagram of the general structure of a hair cell and relevant parameters: hair stiffness $k$, hair length $L$, and base stiffness $S . F$ represents any external force, tactile, viscous, etc. . . . . . . . . . . . . . . . . 4

1.2 Electron microscope image of the hair cell of a frog. Image used under Creative Commons Licenst ${ }^{1}$. . . . . . . . . . . . . . . . . . 6

1.3 Anatomy of the human ear with frequency mapping of the cochlea. Colors denote the primary ear sections: outer ear (green), middle ear (red), inner ear

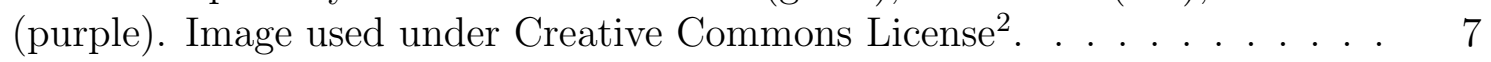

1.4 A) Simplified diagram of a generic lipid molecule. B) Types of lipid formations in aqueous solution. Image used under Public Domain ${ }^{3}$. . . . . . . . . . . . . 12

1.5 Simplified diagram of a droplet interface bilayer (DIB). Lipid molecules form monolayers at the oil-electrolyte interface. Contacting monolayers "zip" to form a bilayer. The aqueous electrolyte can be either in liquid or solid hydrogel form. . . . . . . . . . . . . . . . . . . . . . . . . . . . .

1.6 Two variations of mechanocapacitance. A) Capacitance change due to membrane bending around a planar configuration. Capacitance increases in both directions. B) Capacitance change due to membrane bending around a prebent configuration. Capacitance increases with increased curvature and decreases as membrane becomes planar. . . . . . . . . . . . . . . . . . . 18

2.1 A) A cross-sectional view of substrate chamber housing the hair cell unit shows how solid hydrogels serve to support the hair as well as the lipid lenses that are brought into contact to form a bilayer. The hydrogels were cured onto glass pipettes containing electrodes through which all electrical measurements were made. $L$ indicates the free length of the hair while $h$ indicates the height of the hydrogel base. B) A microscope image taken from underneath the substrate illustrates a typical bilayer formation. . . . . . . . . . . . . . . . . 29 
2.2 A top view diagram depicting how AHC units were arranged into an array. The substrate chambers were machined into a single piece of acrylic with a spacing between hairs of approximately $7 \mathrm{~mm}$. The electrodes were wired so that each bilayer was connected in parallel, essentially forming a larger capacitor. The circuit was voltage clamped and the current output of the system was measured . . . . . . . . . . . . . . . . . . . . . . . . . . . . 31

2.3 A) Low-noise workstation with relevant equipment and Faraday cage shielding. B) Close up image on the microscope table showing the positioning of the micromanipulator and air nozzle relative to the test AHC substrate . . . 33

2.4 Real-time interrogation of the AHC membrane by applying a $10 \mathrm{~Hz}, 20 \mathrm{mV}$ peak-to-peak triangle wave (top) and monitoring current output (bottom). The output square wave magnitude is directly proportional to the bilayer capacitance. The sudden increase in the current output at around 35 seconds reflects a capacitance increase and signals bilayer formation and growth. . . . 34

2.5 A top view of the experimental setup for air pulse excitation shows its relative orientation to the hair cell unit. The air line was split evenly and placed at a distance of $15 \mathrm{~mm}$ from both hair and microphone. The air pulse was aimed at a height $20 \mathrm{~mm}$ above the base of the hair. The air pressure input to the microphone (measured in volts) was compared to the current output (pA) of the voltage clamped hair cell. . . . . . . . . . . . . . . . . . . . . 35

2.6 A base excitation test was performed on hair-hydrogel assembly suspended in air through the use of a modified substrate. No oil or lipids were used for this experiment. The modified substrate was mounted to a modal shaker which was supported by an anti-vibration table. . . . . . . . . . . . . . . . . . . 37

2.7 A top view of the experimental setup for air pulse excitation shows the pulse's relative orientation to the hair cell unit. The air line was split evenly and placed at a distance of $15 \mathrm{~mm}$ from both hair and microphone. The air pulse was aimed at a height $20 \mathrm{~mm}$ from the base of the hair. To create the pulse, the air was pressurized to 40 psi then released rapidly by opening the valve at $0.5 \mathrm{~ms}$ intervals. The air pressure input to the microphone (measured in volts) was compared to the current output (pA) of the voltage clamped hair

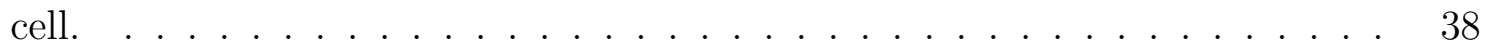

2.8 A top view of the experimental setup for constant airflow excitation shows the arrangement of the array in relation to the air direction. The air source could be moved laterally to change the excitation position. The air source was aimed at height of $20 \mathrm{~mm}$ from the base of the hair at a distance of 15 mm. A mass flowmeter measured the steady-state flow rate of the air. The output current was the result of the free vibration of the hair(s), measured as described in the 2.2 Experimental Measurements section. . . . . . . . . . . . 39 
3.1 Model of the hair-hydrogel system as a uniform, linearly tapered beam with torsional and linear stiffness at the base. . . . . . . . . . . . . . . . . . . . . 42

$3.2 \quad$ Electrical circuit representation of a droplet interface bilayer. . . . . . . . . . 44

3.3 Two variations of mechanocapacitance. A) Capacitance change due to membrane bending around a planar configuration. Capacitance increases in both directions. B) Capacitance change due to membrane bending around a prebent configuration. Capacitance increases with increased curvature and decreases as membrane becomes planar. . . . . . . . . . . . . . . . . . . 45

4.1 Voltage dependence of both types of electrical noise: electrode noise (a) and power-line noise (b). Data is a result of measuring airflow current response (at a constant $34 \mathrm{~m} / \mathrm{s}$ ) of an AHC with no bilayer. Colored lines denote the AHC applied voltage. Dotted lines indicate the experimentally measured natural frequencies of the hair. . . . . . . . . . . . . . . . . . . . . 49

4.2 Air velocity dependence of both types of electrical noise: electrode noise (a) and power-line noise (b). Data is a result of measuring airflow current response of an AHC with no bilayer at $80 \mathrm{mV}$ as the air velocity is increased incrementally from 18 to $45 \mathrm{~m} / \mathrm{s}$ (colored lines). Dotted lines indicate the experimentally measured natural frequencies of the hair. . . . . . . . . . . 50

4.3 Control analysis of the AHC output for bilayer and no bilayer cases as both voltage and air velocity are increased. The root mean square (RMS) amplitude of the current output signal was used a comparison of the overall magnitude of the signal. . . . . . . . . . . . . . . . . . . 52

4.4 Experimental setup for array isolation control experiments. The output of the top AHC was monitored to observe any vibration leakage from the bottom AHC, which was not connect. Three different airflow positions were used: 1) airflow directly at hair 1,2) airflow directly at hair 2 and 3) airflow at both

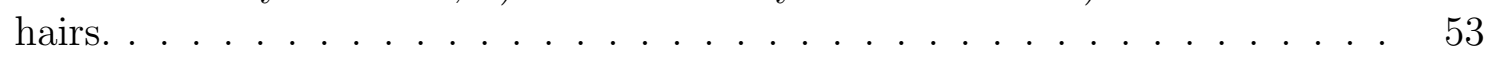

4.5 Vibration isolations test where an AHC output is measured while an adjacent AHC is excited. In this case, the measured AHC was held at $80 \mathrm{mV}$ while the adjacent hair was excited incrementally from 18 to $40 \mathrm{~m} / \mathrm{s}$ airflow. Output shows that small vibrations are picked up only at the third of the adjacent hair's natural frequencies (dotted lines). Refer to Figure
4.4 for more

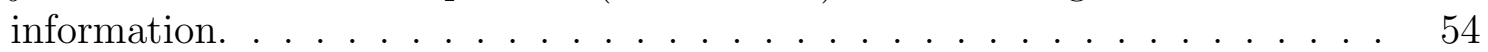


4.6 Vibration isolations test where both a connected and unconnected AHC where excited. In this case, the measured AHC was held at $80 \mathrm{mV}$ while the adjacent hair was excited incrementally from 29 to $40 \mathrm{~m} / \mathrm{s}$ airflow. Output shows that the natural frequencies of the unconnected hair (dotted lines) are overshadowed by the output of the connected AHC. Refer to Figure 4.4 for more information. . . . . . . . . . . . . . . . . . 54

4.7 A) Average spectrum transfer function magnitude (hair velocity ( $\mathrm{mm} / \mathrm{s}$ )/shaker load (V)). Dotted lines show model frequency approximations for $t=0.0018$ $\mathrm{Nm} / \mathrm{rad}$ and $k=39 \mathrm{~N} / \mathrm{m}$ (red), $k=98 \mathrm{~N} / \mathrm{m}$ (black), and $k \approx 200 \mathrm{~N} / \mathrm{m}$ (green). B) Average spectrum phase. C) Coherence plot shows a high signal to noise ratio throughout. . . . . . . . . . . . . . . . 55

4.8 Experimentally determined mode shapes of the hair-hydrogel assembly. . . . 56

4.9 A) Membrane current output versus time after hair pulse excitation (AHC system output). B) Pressure microphone voltage output versus time (AHC system input). . . . . . . . . . . . . . . . . . . . 58

4.10 A) Transfer function estimate (output current (pA)/pulse pressure (V)) for a pulse excitation. Dotted red lines indicate model frequency approximation for $k=19 \mathrm{~N} / \mathrm{m}$ and $\tau=0.0011 \mathrm{Nm} / \mathrm{rad} . \mathrm{B})$ Signal coherence. . . . . . . . . 59

5.1 Measured membrane capacitance versus the applied voltage squared. Error bars denote \pm 1 standard deviation. Capacitance shows a linear increase at low voltages (fit: $C=0.0205 V^{2}+954.8 ; R^{2}=0.95$ ) and plateaus at high voltages (fit: $C=0.0039 V^{2}+993.8 ; R^{2}=0.71$ ). . . . . . . . . . . . 61

5.2 Power spectral density of the AHC output current at various voltages (colored lines) for a constant air flow. The PSD was computed using Welch's method. 62

5.3 Amplitude current density of the AHC versus applied voltage for a constant air flow. Each ASD value is the square root of the PSD integrated over a region \pm $80 \mathrm{~Hz}$ around each frequency. Colored points represent the measured natural frequencies. White dots represent the ASD for an integration over the entire spectrum $(100-2 \mathrm{k} \mathrm{Hz})$ (Total Spectrum Fit: $i_{A S D}=2.2 \times 10^{-5} V^{3}+2.7 \mathrm{~V} ; R^{2} \approx$ $\left.1 ; R_{\text {Linear }}^{2} \approx 1\right)$. . . . . . . . . . . . . . . . . 63

5.4 Power spectral density of the AHC output current at various air flow (colored lines) for a constant $50 \mathrm{mV}$ applied voltage. The black lines show predicted natural frequencies for $k=19 \mathrm{~N} / \mathrm{m}$ and $\tau=0.0011 \mathrm{Nm} / \mathrm{rad}$. The PSD was computed using Welch's method. . . . . . . . . . . . . . . 65 
5.5 Relation between the spectral amplitude and air speed at $50 \mathrm{mV}$ applied potential. Modal frequency amplitudes are shown in color and the total integrated spectrum is shown in white. (fit: $i_{A S D}=-27.5+3.39 A S ; R^{2} \approx 1$ )

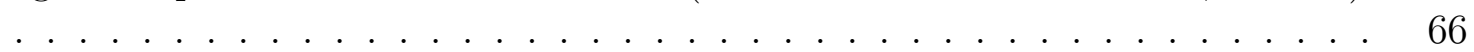

6.1 The test array consisted of three adjacent AHCs of different hair lengths: 33 $\mathrm{mm}$ (AHC 1), $26 \mathrm{~mm}$ (AHC 2), and $20 \mathrm{~mm}$ (AHC 3). The array was excited by sweeping an air source laterally in $2 \mathrm{~mm}$ intervals. The $0 \mathrm{~mm}$ position was slightly to the outside of AHC 1 while the final position was slightly to the outside of AHC 3, a span of $20 \mathrm{~mm}$. The air source was placed at a distance of $15 \mathrm{~mm}$ from the AHC hairs and wide enough to cover a lateral space of approximately $10 \mathrm{~mm}$, enough to excite two hairs at once. The airflow rate was measured through a mass flowmeter and the summed current output was measured through a voltage clamped, parallel circuit configuration. . . . . . 68

6.2 Reference fitting operation for a sample power spectral density of an individual AHC. The blue dots are the experimentally measured PSD values of 5-10 second time signal. The four green lines are the individual peak fits described by a single summation term in equation 6.1 . The red line is the sum of the four green lines, i.e. $R F_{h}(f)$. Note that the average mean squared error in this example is about $3 \% . \ldots \ldots \ldots$. . . . . . . . . . . 71

6.3 Combined fitting operation for a sample power spectral density of the simultaneous output of three AHCs (blue dots). The three red lines are the weighted reference fits for each $\mathrm{AHC}$, that is the function $c_{i} * R F_{i}(f)$ for $\mathrm{AHC}$ $i=1,2,3$. The violet line is the sum of the three red lines, i.e. $C F(c, f)$ from equation 6.2 . Note that one red line is very close to zero, suggesting that the particular reference fit contributed little to the total output. . . . . . . . . . 72

6.4 The HFRD method is applied to the airflow response data from section 5.2 . By self-referencing the method to PSD data at different airflow velocities $(14.2$, 35.6 , and $56.9 \mathrm{~m} / \mathrm{s}$ ), we can create a curve of contribution coefficients. The coefficients $c_{h}$ are then scaled as in equation 6.4 , to convert them to RMS estimates (points in red, blue and green). Notice how the points agree well with the integrated ASD of the same data, another measure of RMS. Also notice the high linearity of the HFRD estimates, shown by the dotted linear fits (red: $R^{2}=0.995$, blue: $R^{2}=0.996$, green: $R^{2}=0.996$. . . . . . . 73 
6.5 Visualization of an HFRD calculation at a measurement instance. The flow is fixed at a position $8 \mathrm{~mm}$ from the starting point. The actual output shown is the PSD of the measured time signal, assumed to be the sum of individual AHCs $\left(i_{1}+i_{2}+i_{3}\right)$. Each current is approximated by its reference fit $R F_{h}$, calculated before the test (see Figure 6.2). The reference fits are scaled by the contribution coefficients $\left(c_{h}\right)$, which represent the relative level of excitation that each AHC is receiving at the particular airflow location. The sum of the terms (see Eq. 6.2) results in the combined fit $C F(c, f)$ which is an approximation of the actual output. The precise values of $c_{1}, c_{2}, c_{3}$ are chosen by a fitting algorithms which minimizes the error between $C F(c, f)$ and the actual output. . . . . . . . . . . . . . . . . . . . . . . . . . .

6.6 Sensitivity of the $c_{h}$ coefficients to applied air velocity for an array of three AHCs. The circular data points indicate the calculated reference values at approximately 12.7 and $25.4 \mathrm{~m} / \mathrm{s}$. The dotted lines indicate the estimated linear sensitivity curve from a fit of equation 6.8 . The $\beta_{h}$ sensitivity values (slope of the line) are labelled next to each curve: $\beta_{1}=0.119 \mathrm{~m} / \mathrm{s}^{-1}, \beta_{2}=$ $0.085 \mathrm{~m} / \mathrm{s}^{-1}$, and $\beta_{3}=0.115 \mathrm{~m} / \mathrm{s}^{-1}$. . . . . . . . . . . . 76

6.7 Nominal airflow sensitivity measurements of AHC reference data (red circles) show that the average sensitivity increases with hair length. Standard deviation error bars indicate no significant difference between the two lowest length. Normalizing the nominal data the bilayer capacitance tended to reduce the standard deviation, except for the $26 \mathrm{~mm}$ hair, suggesting that bilayer area also has an effect on sensitivity. . . . . . . . . . . . . . . . . . . . 77

6.8 Illustration of the airflow mapping process using HFRD for a flow postioned at $8 \mathrm{~mm}$ with a magnitude of $19.32 \mathrm{~m} / \mathrm{s}$. A) The PSD of sensor data (blue dots), with the air source position on $8 \mathrm{~mm}$, is fitted using the HFRD method to obtain the $c_{h}$ coefficient values. B) The $c_{h}$ values are mapped to their respective positions, indicating the relative excitations of the AHC sensors. C) Sensitivity estimates for each of the three AHC provide a way to convert the $c_{h}$ values to an air velocity reading. D) $c_{h}$ values, converted to velocity estimates using the sensitivity curves from (C), have been mapped once again to their spacial locations. This provides a visualization of the estimated velocity profile for an air source positioned at $8 \mathrm{~mm}$. . . . . . . . . . . . . . . . . . 79 
6.9 Hair frequency response decomposition of an array output signal shows the excitation levels of the three AHC components (red, blue and green curves) as an air source is moved incrementally across (step size of $2 \mathrm{~mm}$ ). The labels at the top of the graph indicate the position of each AHC hair $(2,10$ and $18 \mathrm{~mm})$. The horizontal axis indicates the position of the center of the air nozzle, i.e. the position of maximum air velocity. The air flow profile in this case had a maximum velocity of $20.7 \mathrm{~m} / \mathrm{s}$, indicated by the dotted black line. The estimated air velocity values are based on linear interpolation of two reference points, and in ideal conditions should peak at the maximum applied velocity. The discrepancies observed here, particularly in AHCs 1 and 3, suggest instabilities in the sensitivities of the bilayer membranes. . . . . . 81

6.10 Two examples of experimental deviations from the simplifying assumptions of the HFRD method. A) The top figure shows two output PSD responses of AHC 2 at $12.7 \mathrm{~m} / \mathrm{s}$ with the same bilayer, taken before and after an airflow test, approximately 30 minutes apart. A magnitude decrease of $74 \%$ is observed. On the bottom, we see the respective bilayer triangle wave time responses, whose magnitudes are directly tied to the capacitance and area of the bilayer. A decrease of about $69 \%$ observed in this case indicates a similar decrease in bilayer size. B) The output PSD responses of two successive tests at 12.7 and $25.9 \mathrm{~m} / \mathrm{s}$ demonstrate a change in relative modal values, most noticeably between the first and fourth output modes. . . . . . . . . . . . . . 82 


\section{List of Tables}

$5.1 \quad$ Eqn.|5.3| fit parameters for voltage sensitivity of each natural frequency. . . 64 


\section{Chapter 1}

\section{Introduction and Literature Review}

Over millions of years of design iteration and natural selection, evolution has produced many specialized sensory organs capable of outperforming current state of the art engineering devices. Compound eyes, for example, enable flies to navigate complex environments with agility. The best cameras are still unable to demonstrate such performance at a comparable size scale, especially with the limited processing resources available in the fly's nervous system [1, 2]. Human ears, too, display a combination of dynamic range, frequency response and sensitivity unmatched by any single microphone or acoustic sensor [3, 4]. Biological sensors are robust, adaptable and even regenerative. They also demonstrate an impressive ability to miniaturize effectively and efficiently. Even at the cellular level, biomolecular sensors exist, capable of detecting mechanical, optical and chemical stimuli at atomic scales [5] [6].

In recent years, engineering have turned to biological studies of sensory organs for inspiration toward the development of advanced technologies. The diversity of biological sensors provides a wealth of ideas for transformation, transduction and processing of environmental stimuli. These concepts, however, are most often translated into traditional silicon-based technologies. While current engineering knowledge allows for more rapid development of electronic components, miniaturization is increasingly limited by instabilities at the quantum scale [7]. Biomolecules, on the other hand, operate effectively at this scale. Engineers should consider not only "bio-inspiration," but also "bio-incorporation," the development of technologies based on biological components.

By following this philosophy, this work seeks to advance the development of smart materials based on biomolecular transduction. The scope focuses primarily on flow sensing, although 
it is hoped that the finding will have a broader impact. Biological hair cells, which are found across the animal kingdom for a range fluid sensing purposes, are our source of bioinspiration. In terms of transduction, we explore the capabilities of the lipid bilayer. Lipid bilayer are the basic building unit for all biomolecular transduction, and as such provide an excellent starting point for bio-incorporation technology. Based on these principles, we aim to develop artificial hair cell arrays that share key properties with their biological counterparts and use this as a platform for learning more about biology as a whole.

\subsection{Biological Hair Cells}

The hair cell is a biological sensor that uses microscopic hair-like structures to detect delicate motions of surrounding fluid. Most hair cells range from tens to thousands of micrometers in length and exhibit sensitivities to excitation energies as low as $10^{-10} \mathrm{~J}$ [8]. In addition, they can detect a wide range of stimuli from a similar basic structure. Insects [9, 10, fish [11, and mammals [12, 13] have evolved sensory organs that utilize hair cells to sense flow, sound, vibrations and even gravity [8]. Function is primarily determined by the cell's surrounding medium, its supporting structure, and its organization within an organ. Organisms almost always employ hair cells in large numbers, synthesizing their neural signals to acquire sophisticated sensory information. The blind cave fish can navigate obstacles in complete darkness by detecting flow fields around its body [14, 15] and mammalian ears can capture dynamic range of frequencies as wide as 20-100,000 Hz via spacial mapping of hair cells along the cochlea [12, 3, 6]. From an engineering perspective, the sensitivity and adaptability of hair cells make them an ideal inspiration for small, modular, multi-purpose sensors. This introduction to hair cells will begin by elaborating on the basic mechanics of their general biological structure and present natural examples with major relevance to this study.

\subsubsection{Basic structure and mechanics}

Hair cells share several fundamental structural and operational traits across all animal species. The great diversity in their biological sensory function can be accounted for through changes in specific geometric and material parameters and the mechanics of peripheral structures. Strictly speaking, hair cells are specialized sensory cells that exist only in vertebrates. Microscopic rods, called stereocilia, extend from the top surface of the cell and interact with 
the outside environment [13, 12]. The hair-like nature of the stereocilia gives rise to the name "hair cell." However, in this work we will use the term hair cell more broadly to include all biological units that use a hair-like structure as a sensing element, including actual sensory hairs on some insects. All these units still share important engineering principles of hair cells.

The use of hair-like structures as mechanical sensing is ubiquitous in nature and can be generalized by a few basic parameters as show in Figure 1.1. In its most basic form, the hair cell is a lever arm coupled to a strain sensor which detects the arm's rotation or displacement. An external force deflects the hair and the strain sensor, typically a set of mechanosensitive channels, trigger a neuronal signal based on the deflection. The key mechanical parameters in this coupling are the hair stiffness $k$, hair length $L$, and base stiffness $S . L$ is clearly a geometric property and $k$ and $S$ depend on the material properties of the hair and its supporting structure. The values of $k, L$, and $S$ can be adjusted for optimal response to environmental forces. For flow sensing, these forces depend primarily on the physical properties of the surrounding fluid (density, viscosity, etc.) [16]. Furthermore, the hair cell parameters can even be fine tuned to particular frequencies of interest to the animal. In almost all flow-sensing biological hair cells, $k$ is much larger than $S$, producing a rigid hair motion [17, 18, 16]. This may help to eliminate bending modes in oscillatory flows, simplifying the dynamics.

As will be demonstrated in the next section, variations on the basic structure do occur in nature, although the operational principles are similar from species to species. Instead of stiffening the material, for example, multiple hairs may be coupled together to increase the overall stiffness of the structure. Such adaptations, and many others, help to illustrate the simplicity and modularity of the hair cell in an engineering context. 


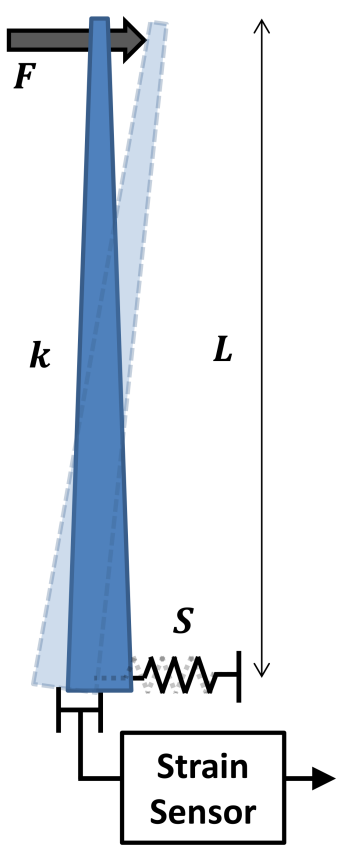

Figure 1.1: Diagram of the general structure of a hair cell and relevant parameters: hair stiffness $k$, hair length $L$, and base stiffness $S . F$ represents any external force, tactile, viscous, etc.

\subsubsection{Hair cell diversity in nature}

In order to illustrate the complexity and functional diversity that may be achieved through combinations of basic hair cell structures, we survey some key examples in the natural world. Since the research interest of this work is flow sensing, the examples focus on this particular application of biological hair cells as well. In addition, details are concentrated on the physical mechanics of the biological systems in order to emphasize the key design principles in hair cell sensing. We begin with the small sensory hairs of arthropods and move to vertebrate auditory hair cells and fish lateral lines, all crucial biological systems that help animals interact with their environment.

\section{Spider Trichobothria}

The arthropod exoskeleton is lined with hundreds of thousands of mechanosensitive hairs, referred to as hair sensilla [10, 19]. For terrestrial arthropods, such as spiders, hair sensilla consist of extremely fine hairs suspended by a soft joint membrane within sockets of the hard 
exoskeleton, primarily on their legs. A sensory dendrite at the hair shaft's base responds to the angular deflection of the hair, sending impulses to the central nervous system [18]. Remarkably, through changes in only a few geometrical and mechanical parameters of this basic structure, hair sensilla can greatly alter their sensitivity and even function [18, 17]. In fact, spider hair sensilla can be separated into two main types, based primarily on the relative stiffnesses of the hair and supporting joint membrane: tactile sensors and flow sensors, also called trichobothria. Tactile sensors respond to touch and aid the spider in navigation. For these sensors, the base restoration stiffness and hair stiffness are similar, so the hair undergoes both rotation and bending under an applied force. The bending behavior helps to dissipate some of the force and avoid overloading the sensor. Alternatively, trichobothria respond to minute air flows around the spider's body and are used for predator and prey detection. These hairs do not bend, since their base stiffness is low compared to the hair stiffness [17]. They rotate only at the base and respond to flow velocities. Trichobothria are some of the most sensitive sensors in the animal kingdom, requiring only about $10^{-10} \mathrm{~J}$ of energy to trigger a neural signal [20, 10]. This corresponds to air motions of roughly $1 \mathrm{~mm} / \mathrm{s}$ [18].

Spiders possess close to a thousand trichobothria altogether [17]. They are grouped in clusters of about five hairs, ranging in length from 100 to $1400 \mu \mathrm{m}$. The trichobothria length determines its resonance frequency, turning each cluster into a set of bandpass filter, typically in the range of 40-600 Hz within length differences less than $1.5 \mathrm{~mm}$. Similar cluster occur throughout the body of the spider, giving the animal the ability to discriminate magnitude, direction, frequency and variation in space of surrounding airflows [18, 10, 21].

\section{Hair Cells in Vertebrates}

Another class of hair cells occurs in virtually all vertebrates, from fish to reptiles to mammals, with an essentially identical structure. The uses of the cells, however, can vary significantly: they can help in sensing aquatic disturbance or in detecting and interpreting sound. Figure 1.2 shows the hair cell of a frog, which helps to illustrate the properties of general vertebrate hair cells. A flat cell surface flexibly supports a bundle of closely packed stereocilia, the "hairs" in this case. The bundle is hexagonal in shape, with the stereocilia arranged from shortest to longest along a single axis, forming a mirror-symmetric pattern [13, 12, 3]. Individual stereocilia are only a few micrometers tall and a few hundred nanometers in di- 


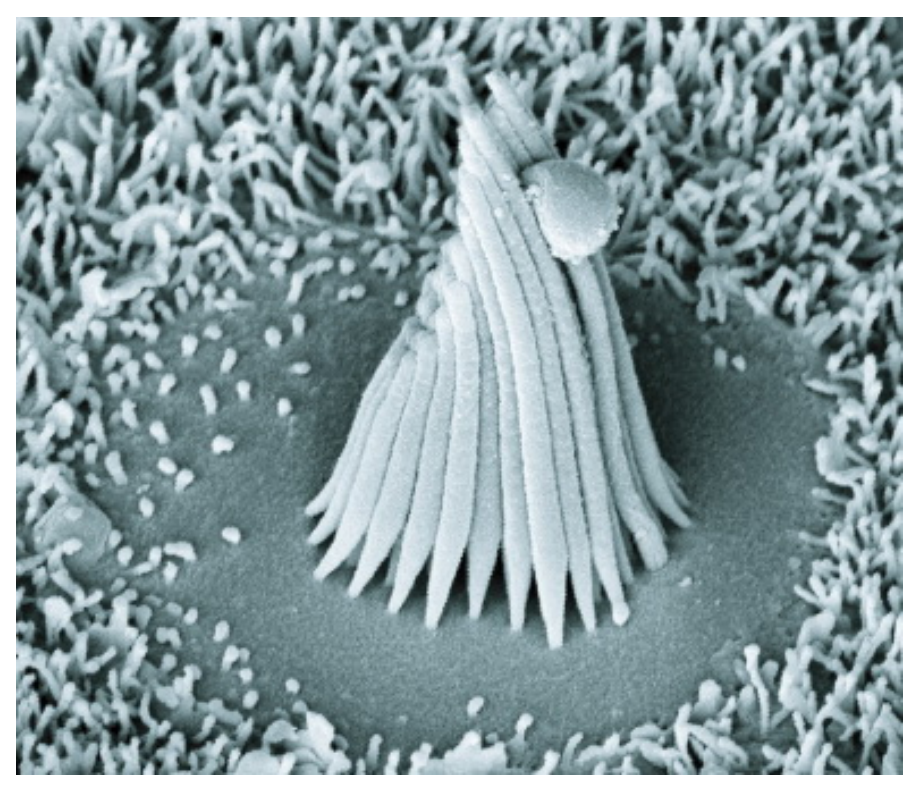

Figure 1.2: Electron microscope image of the hair cell of a frog. Image used under Creative Commons License ${ }^{1}$.

ameter [12]. Adjacent stereocilia are connected by small fibers called tip links, which run in the direction of the symmetric axis. This makes hair bundles stiffer to deflection along this axis than perpendicular to it [13, 3]. When the hair bundle deflects under an applied force, it bends only at its base [13]. Hair cells have been experimentally shown only to respond to deflection along the symmetric axis. In some undisturbed hair cells, approximately $15 \%$ of transduction channels are open. Deflection in the direction of increasing stereocilial length will open more channels depending of the size of the stimulus, increasing the electrical response. A stimulus in the opposite direction will decrease channel activity. A hair bundle need only be displaced by $\pm 0.3 \mathrm{~nm}$ at the tip to reach the threshold of hearing. This corresponds to only a motion of $\pm 0.003 \mathrm{deg}$ [12]. The precise connection between stereocilia displacement and channel activity has not yet been uncovered. However, tip links are thought to act as gating springs for the mechanosensitive channels within hair cells. A positive stimulus increases tension in the springs and promotes channel gating [13, 12]. In essence, then, the vertebrate hair cell is a type of biological strain gauge with remarkable sensitivity properties, both in magnitude and direction.

\footnotetext{
${ }^{1}$ By A. James Hudspeth, M.D., Ph.D. (Personal communications) [CC BY-SA 3.0 (http://creativecommons.org/licenses/by-sa/3.0)]
} 


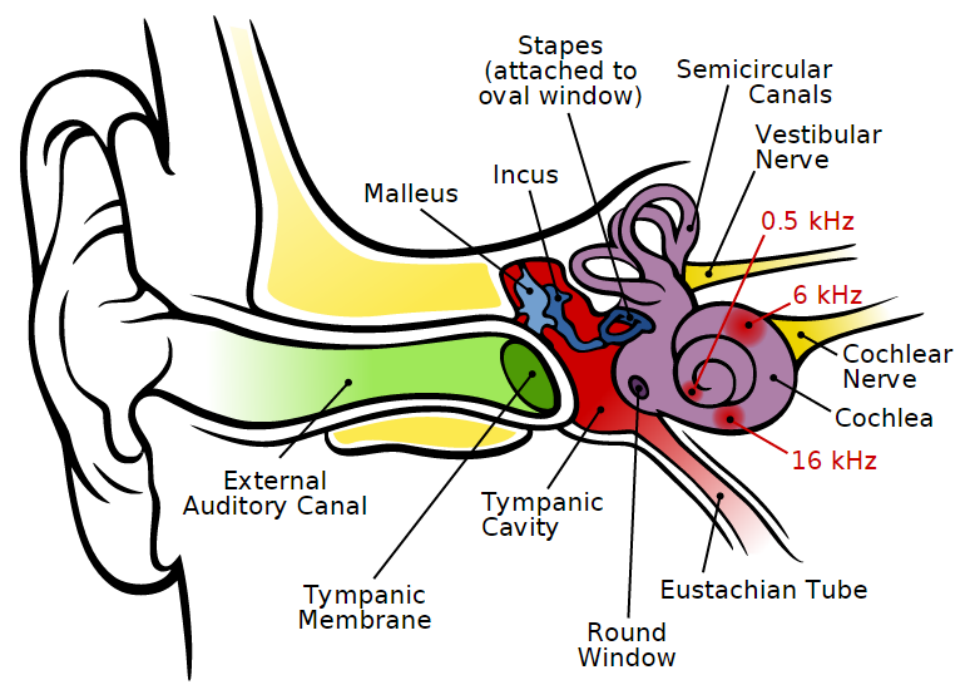

Figure 1.3: Anatomy of the human ear with frequency mapping of the cochlea. Colors denote the primary ear sections: outer ear (green), middle ear (red), inner ear (purple). Image used under Creative Commons License ${ }^{2}$.

\section{Auditory Cochleae}

Specialized, flow-sensing hair cells called inner hair cells are critical for vertebrate audition. In general, the vertebrate auditory system is composed of the outer ear, the middle ear and the inner ear. Figure 1.3 uses the human ear as a characteristic example. In the outer ear (shown in green), sound enters as variations in air pressure through an ear canal and resonates the tympanic membrane, or eardrum. In the middle ear (shown in red), tympanic membrane oscillations are mechanically transferred to the cochlea via a linkage of tiny bones called ossicles. The cochlea, a fluid-filled, spiral cavity which is responsible for processing the spectral characteristics of sound, is part of the inner ear (shown in purple). Hair cells in the cochlea activate due to fluid motion and this information travels to the brain through a complex series of auditory nerves [3].

The cochlea is a marvel of biological engineering, and understanding its mechanics is essential for grasping the sensory potential of hair cells. The organ consists of three fluid-filled chambers which span its entire length: the scalae vestibuli, tympani and media. The scalae

\footnotetext{
${ }^{2}$ By Inductiveload [CC BY-SA 2.5 (http://creativecommons.org/licenses/by-sa/2.5)], via Wikimedia Commons
} 
vestibuli and tympani are filled with perilymph liquid. The fluid is displaced when the foot of the last of the ossicles, the stapes, pushes on a membrane-covered window at the base of the scala vestibuli, called the oval window. The fluid movement in the scala vestibuli communicates with the scala tympani at the apex of cochlea through the heliocotrema [3, 22]. A membrane-covered window at the base of the scala tympani, acts as a pressure release. The scala media rests along the center of the cochlea, separated from the scala vestibuli by Reissner's membrane and from the scala tympani by the basilar membrane. The organ of corti rests on the basilar membrane, lined by a single row of inner hair cells. Hair bundles, called sterocilia, extend from the inner hair cells into the endolymph fluid of the scala media. Pressure waves reaching the oval window produce pressure differences among the three scalae, causing motions of the basilar membrane. The friction of the endolymph fluid against the inner hair cell stereocilia causes them to deflect. The deflection triggers the activation of mechanosensitive channels in the cell membrane, which in turn send electrical impulses to the brain. [3, 22, 23, 24]

A single human cochlea contains approximately 16,000 hair cells [12]. In order to interpret the spectral characteristics of sound, the cochlea performs a mechanical Fourier analysis, mapping input frequencies to specific locations along its length [22] [25]. It has been shown that tonal input causes waves to propagate through the basilar membrane. The location of peak amplitude is a function of the input frequency; high frequencies peak near the base of the cochlea while low frequencies do so near the apex [22]. An example of the cochlear frequency mapping is illustrated in Figure 1.3. This phenomenon is linked to the unique mechanical properties of the basilar membrane. Its stiffness decreases by several orders of magnitude with distance from the base of the cochlea. The variation in stiffness along the basilar membrane has been correlated to the distribution of characteristic frequency (most sensitive frequency) in the cochlea [22] 26]. The inner hair cells that line the basilar membrane (via the organ of corti) transduce its mechanical motion in a discrete fashion. In this way, a single frequency stimulus elicits an electrical response at a specific cochlear location. The complex mechanical interaction within cochlea is what endows mammals to hear and impressive range of frequencies: $20 \mathrm{~Hz}$ to $20 \mathrm{kHz}$ in humans and more than 100 $\mathrm{kHz}$ for other mammals [22]. 


\section{Fish Lateral Lines}

Another specialized use of hair cells occurs in the lateral lines of many aquatic vertebrates, especially fishes. The lateral line consists of rows of small sensory organs, called neuromasts, distributed along the trunk and around the eyes and jaw of the animal. Lateral lines allow fish to detect flow fields around their bodies, a sense that has been described as "touch at a distance [27]." Studies have demonstrated that lateral lines play a role in complex aquatic behaviors such as schooling [28], prey detection [29], courting [30], station holding (swimming in place) [31, and rheotaxis (turning to face an incoming flow) [32]. Depending on the behavior, fish can engage in either active or passive sensing. In the former, a swimming fish uses distortions in a self-generated flow field to detect an obstacle's presence. In the latter, a stationary fish senses external flow fields created by other moving bodies.

Neuromasts, the basic sensory organs of the lateral line, are a combination of hair cells, very similar in structure to those found in the vertebrate auditory system described above [33]. In fact, significant portions of our current knowledge about neuromast behavior originate from the study of inner ear hair cells [16]. Key differences between lateral line and auditory hair cells are found in their organization and peripheral organs. Hair cells are grouped via a solid, gelatinous dome called the cupola which can range from about $30-600 \mu \mathrm{m}$ in diameter, depending on the function of the neuromast [8, 33]. Cupolas couple the deflection of the hair cells inside. They are the part of the neuromast that is in contact with the water around the fish. Cupolar displacement alone responds mainly to the velocity of a water flow, as it is driven mainly by viscous force The cupola moves as a rigid body, creating shear at its base and the bases of the attached stereocilial bundles and prompting them to produce neural impulses [8]. The sensory axis of the hair cells within the cupola follows the lateral line. They are divided into two groups of opposite polarities, innervated by separate sets of nerve fibers. This produces bidirectional sensitivity along the later line and none perpendicular to it [33, 16]. The threshold of sensing for lateral line hair cells is less than $1 \mathrm{~nm}$ displacement and saturation occurs above $100 \mathrm{~nm}$ [33. However, the sensitivity of particular neuromasts depends primarily on their size and quantity of hair cells. Here, the stiffness of hair cell bundles plays a key role, since their number, along with the cupola size, will determine the total mechanical stiffness of the neuromast. The number of hair cells within a neuromast can vary significantly, from only a few to nearly 3000. When coupled with the fluid dynamics of the surrounding water, changes in stiffness may shift neuromast mechanics from primarily viscosity-driven (small, soft cupolas) to inertia-driven (large, stiff cupolas). Coombs et al. 
have theorized that certain large neuromasts may even be tuned to specific frequencies, presumably to identify the motions of particular prey [16, 34].

Neuromasts can be divided into two sensing categories: superficial and canal neuromasts. Superficial neuromasts are present on the skin of the fish and protrude directly into the water. Canal neurmasts are situated in narrow canals beneath the skin which open to the outside through series of pores. A single canal neuromast occurs between each pair of pores [8, 33, 16, 34]. Because they are smaller in size, the mechanics of superficial neuromasts make them responsive to viscous forces. The result is a type of low-pass filter system that responds best to slow and steady flows around the fish. Montgomery et al. demonstrate the importance of superficial neuromasts in determining the general direction of current flow in surrounding water for behaviors such as rheotaxis [32]. Canal neuromasts are usually larger and stiffer and their response is heavily tied to the mechanics of their enclosing canal structure. Pressure differences between pores, caused by velocity gradients in the external water, drive fluid motion within the canal. This makes canal neuromasts sensitive to ambient water accelerations, allowing them to process transient, high-frequency disturbances, even in the presence of steady background flow [35]. They are typically associated with schooling and prey detection. In addition, the dynamics in adjacent canal segments are independent, providing each canal neuromast with a concentrated spacial sampling of the external flow field [16, 34].

\subsection{The Lipid Bilayer}

Formal study of bilayer lipid membranes has been around since the early 1990s. As the fundamental component of cell membranes, it is arguably one of the basic elements of life. Increased research during the second half of the 20th century saw scientist develop a variety of ways to create artificial lipid bilayers in vitro. The bilayer's ability to self assemble, combined with its uses as a platform for cellular-scale sensing has led to its emphasis as a biological smart material. There has been considerable recent interest in the use of lipid bilayer for versatile, molecular level sensing applications. 


\subsubsection{Structure and biological function}

Lipid bilayer membranes are the foundation of the cellular membrane, one of the most vital structural and functional components of the cell itself. Depending of the role of the cell, the lipid bilayer can be used or modified to perform a number of important tasks [36, 7]:

1. Structural support - enclose the cell cytoplasm, protect the cell organelles and give the cell its shape.

2. Selective barrier - allow only specific molecules to pass in and out of the cell

3. Conduit for transport - house embedded proteins that facilitate the transport of ions, energy, electrons, etc.

4. Receptor -be receptive to various type of stimuli (light, chemicals, tension, etc.). Some are processed through special embedded receptor proteins, while others (e.g. tension) are thought to affect the lipid bilayer membrane itself.

5. Contact - mediate contact with other cells

In many ways, the lipid bilayer or cell membrane can be considered an advanced sensor platform. It forms the basis for how organisms, including humans, interact with the environment [7].

Lipid bilayer are constructed from basic biomolecules called lipids. These are amphiphilic in nature, consisting of two parts: a polar, hydrophilic head group and a non-polar, hydrophobic hydrocarbon chain tail group (Figure 1.4A). When dissolved in an aqueous solution, lipids self assemble into larger cylindrical structures . This self-assembly is driven by the hydrophobic effect, the inability of the hydrocarbon tails to bond with water. As they assemble, the lipid tails bond non-covalently to expel water between them and achieve a more favorable energy configuration. The assembly structures may take different forms, among them micelles, vesicles and sheets, as in Figure $1.4 \mathrm{~B}$. A flat sheet form is typically referred to as a lipid bilayer membrane. It consists of two thin parallel films of lipid molecules, each one molecule thick. The lipid hydrophobic tails face inward, while the hydrophilic heads face outward to contact the aqueous solution. The single films which make up half of the bilayer are referred to as monolayers. Many types of lipids exist, and some are interchangeable. The 

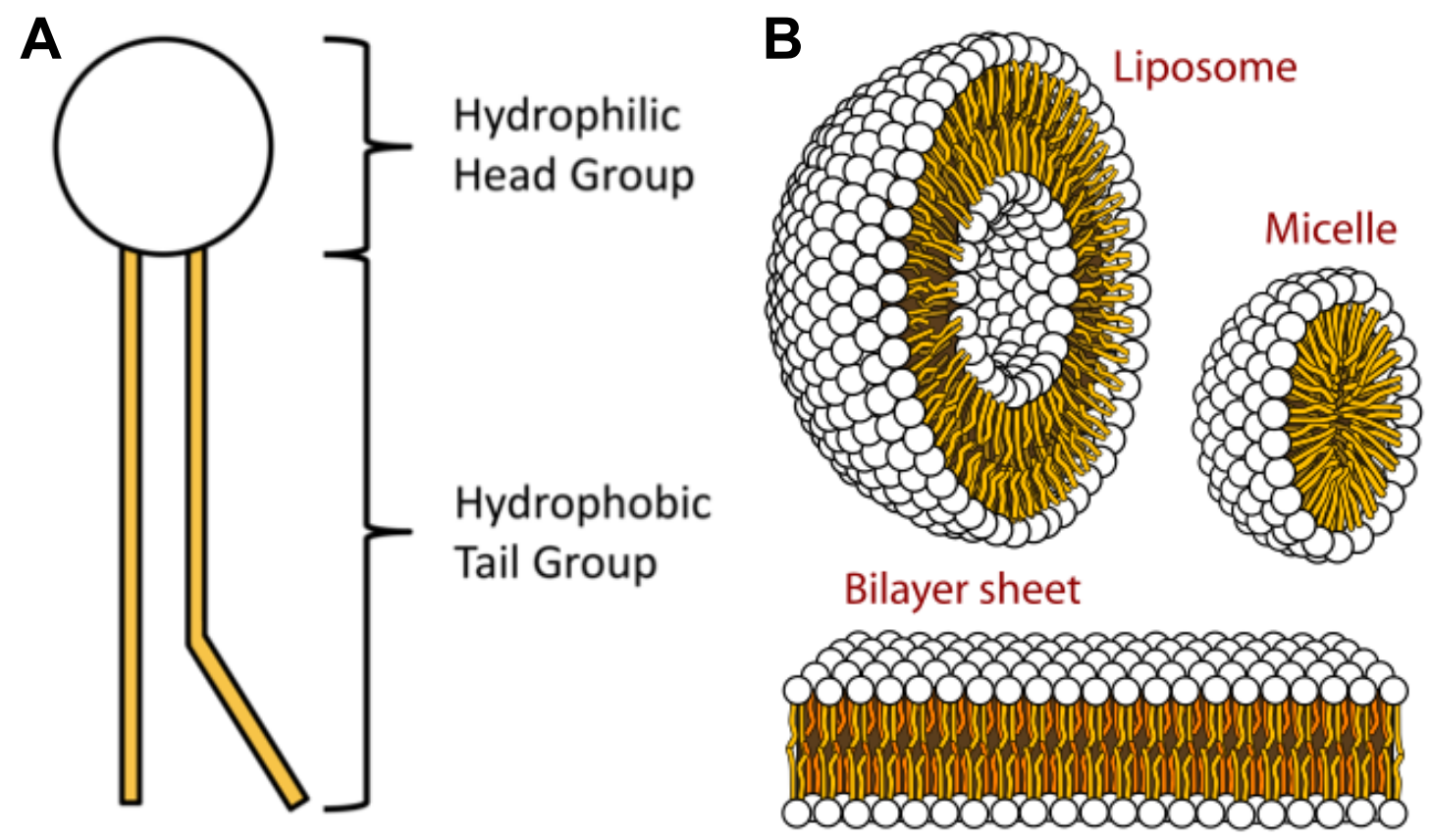

Figure 1.4: A) Simplified diagram of a generic lipid molecule. B) Types of lipid formations in aqueous solution. Image used under Public Domain ${ }^{3}$

basic structure of the bilayer, however, remains consistent across nature. Biological bilayers within cell membranes can range 2-5 $\mathrm{nm}$ in thickness [7, 37, 36].

A pure lipid bilayer is impermeable to all ions (e.g. $\mathrm{Na}^{+}, \mathrm{K}^{+}, \mathrm{Cl}^{-}$), but allows some molecules through, such as water. In the cell membrane, however, the lipid bilayer contains large numbers of special proteins which regulate ion flow and other processes. The cell uses these proteins to communicate with the extracellular environment and other cells [7, 36].

\subsubsection{Formation of Synthetic Bilayers}

Several techniques for forming synthetic lipid bilayers have been developed since the 1960s. Although clear differences between synthetic lipid bilayers and biological cell membranes do exist, studies have shown considerable similarities in thickness, surface tension, and composition [38]. Just like in cell membranes, biomolecules in a lipid bilayer are fluid in the two lateral dimensions and readily accept membrane proteins [39]. Formation methods generally

\footnotetext{
${ }^{3}$ By Mariana Ruiz Villarreal (Own work) [Public domain], via Wikimedia Commons
} 
take advantage of lipids' amphiphilic properties and ability to self assemble. In many cases, synthetic substrates are used to support and encourage bilayer growth. Being a chemically driven process, it is very sensitive to the surface chemistry and geometry of the substrate material. Material choice and support geometry affects the bilayer assembly, stability and the types of procedures that may be performed. Various kinds have been used: steel wire loops [40], porous polycarbonate or glass fiber [41], teflon sheets with apertures [42], and planar gold or glass [43].

Unsupported or free-standing membranes are open to aqueous solution on both sides and are typically formed across an open pore in a material. Lipids are fluid in this configuration, thus inserted proteins are fully mobile and active [44]. The membranes themselves, however, are typically less stable and robust than supported membranes. Supported membranes are those that are contacted on one face by a substrate. These make bilayer more accessible to experiments and imaging but may produce unwanted effects. Chief among them is the mechanical, chemical and electrical coupling that occurs between bilayer and substrate. Mechanically, the membrane's motion is restricted and thus lipid lose their natural fluidity. Chemically, inserted proteins may interact with the substrate and become immobile. Electrically, substrates can change conductive paths through the membrane, so added electrical contributions must always be taken into account. In addition, substrates may have limited compatibility between experiments [39, 45, 46, 38].

The most commonly used formation techniques (both supported and unsupported) are lipid painting, lipid folding, Langmuir-Blodgett/Langmuir-Schaefer, vesicle fusion and interface bilayers.

\section{Lipid Painting}

Lipid painting is perhaps the simplest method of bilayer formation and takes full advantage of lipid self assembly. A brush is used to spread lipids, dissolve in organic solvent, onto a hydrophilic substrate (e.g. glass) with one or various small apertures. The solvent evaporates, leaving behind a film of lipids. When submerged in an aqueous medium, the hydrophobic effect drives bilayer formation on the substrate. An unsupported lipid bilayer is formed in each of the apertures. The lipid painting method is advantageous because of its simplicity, but left over solvent can reduce the stability and quality of the bilayer [39, 46]. 


\section{Lipid Folding}

Lipid folding is also recognized as the Montal-Mueller technique [42]. In this method, two compartments are filled with an aqueous solution, separated by a hydrophobic substrate with a circular aperture. The water level begins below the aperture. Lipid solution is distributed on the top of the aqueous medium, so that a lipid monolayer forms (tails up) on the air-water interface. Then, the water levels are raised simultaneously so that the lipid tails "fold" up onto the substrate and across the aperture. This again qualifies as an unsupported bilayer. Lipid folding experiments have achieved bilayer across a wide range of aperture diameters, $0.2 \mu \mathrm{m}$ to $3 \mathrm{~mm}$.

\section{Langmuir-Blodgett/Langmuir-Schaefer}

The Langmuir-Blodgett/Langmuir-Schaefer method, also calles LB/LS, is essentially the reverse of lipid folding. A lipid solution is spread on an aqueous medium so that a monolayer forms on the surface, tails up. A hydrophilic slide, typically glass, is raised vertically through the air-water interface so that he lipid heads attach to the slide on both sides. In the LB method, the slide would be subsequently dipped down through the interface once more, to create lipid bilayers on both sides by a reverse folding action. In the LS method, the slide is tilted 90 degrees and pushed horizontally through the interface. Both methods result in supported bilayers. They are highly repeatable but have been shown to produce lower quality bilayers than lipid painting [47]. Incorporating proteins is also difficult [43].

\section{Vesicle Fusion}

Vesicle fusion is derived from the cellular method used to package and deliver proteins to the plasma membrane. It requires the formation of vesicles in water through one of several laboratory techniques. After, they are formed, vesicles are transferred to hydrophilic substrate, where they rupture and fold onto themselves, eventually settling into a supported bilayer. This method has proven to be effective for incorporating proteins [39, 46]. 


\section{Interface Bilayers}

Newer methods of bilayer formation have helped ro bridge the gap between the advantages of supported and unsupported bilayers. These methods, generally referred to as droplet interface bilayers or hydrogel-on-hydrogel, rely on an oil-water interface to promote lipid self assembly. Use of the aqueous medium itself as the support provides stability and robustness similar to that of supported bilayers while avoiding synthetic substrates that may interfere with the bilayer mechanics.

Droplet interface bilayers: To form a droplet interface bilayer (DIB), a lipid monolayers are assembled on the surfaces of a aqueous droplets submerged in oil. The interface between the polar aqueous phase and the non-polar oil phase is a natural region for the lipid to assemble. The droplets are then brought into contact to initiate a "zipping" process whereby monolayers push together, expelling the oil between them, and form a bilayer on the droplets' contact area as shown in Figure 1.5. Lipids can be inserted either into the aqueous phase (lipid-in method) or the oil phase (lipid-out method). Proteins dissolved in the aqueous phase will also self-insert to the bilayer [48, 49, 46]. Holden et al. have created DIBs fluid and robust enough to last for days [50]. The major disadvantage of the method is that manipulation of droplets and electrode insertion tends to be more difficult than for fully supported membranes.

Hydrogel-on-hydrogel bilayers: Hydrogel-on-hydrogel bilayers utilize a similar formation concept as DIBs, however, the aqueous phase is replaces by a solid hydrogel for added support an maneuverability. Lipid monolayers will self-assemble on a hydrogel surface though various methods:

1. the lipid-out method, where solid hydrogels are inserted into lipid-filled oil [51, 45]

2. the lipid-in method, where aqueous hydrogels form a DIB and are then cured solid [52]

3. lipid-coating solid hydrogels in oil [53]

Hydrogels make the manipulation of oil-water interface bilayer less difficult. However, the bilayer is not as fluid as it would be in an aqueous droplet, so stability can be affected. The self-supported nature of these interface bilayer has made them amenable to the formation of large, interconnected networks [51]. 


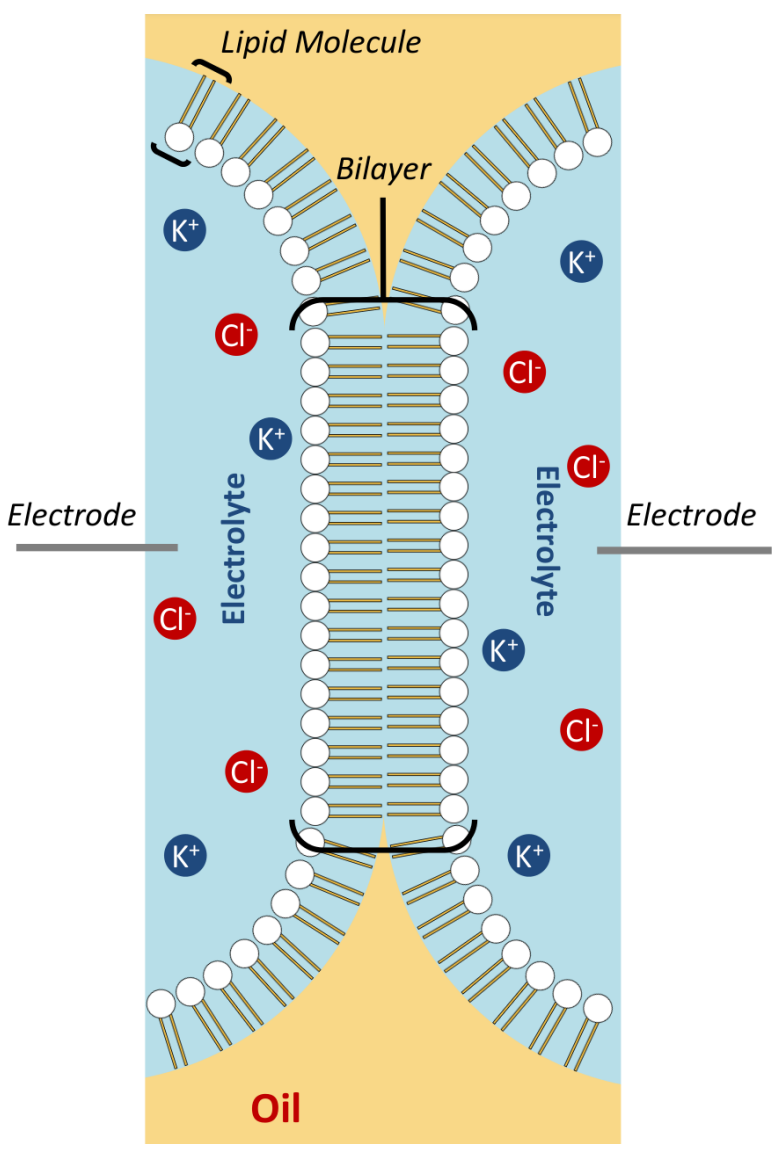

Figure 1.5: Simplified diagram of a droplet interface bilayer (DIB). Lipid molecules form monolayers at the oil-electrolyte interface. Contacting monolayers "zip" to form a bilayer. The aqueous electrolyte can be either in liquid or solid hydrogel form.

\subsubsection{Bilayer Mechanoelectrical Transduction}

The bilayer plays a key sensory role within the cell, which includes transduction between many domains (chemical, optical, etc.). Here, will focus on its ability to respond to mechanical stimuli by altering its electrical characteristics, a quality commonly referred to as mechanoelectrical transduction. Mechanical response is usually tested by applying pressure across the membrane in order to induce a curvature. This is only possible in free-standing or unsupported membranes [54]. Four types of bilayer mechanoelectrical transduction have been postulated, each of which depends on either membrane curvature or lateral stretching:

1. Mechanocapacitance: curvature-induced capacitance change due to bilayer area change. 
2. Electrowetting: tensioning of bilayer due to an applied potential.

3. Flexoelectricity: curvature-induced membrane polarization.

4. Mechanoconductance: increase in ionic conductance due to bilayer stretching.

Mechanocapacitance is the relationship between mechanical bending of the bilayer membrane and its capacitance. It was first observed by Ochs et al. on an edge-supported aperture bilayer, when the researchers accidentally noticed that room vibrations elicited a current response. The effect is an intrinsic property of lipid bilayers; it occurs without the need for embedded proteins that might otherwise react to mechanical stimuli. An undisturbed, planar bilayer can be treated as a parallel plate capacitor, whose capacitance $C$ is defined by:

$$
C=\epsilon_{0} \epsilon A / d
$$

where $\epsilon_{0}$ is the permittivity of free space, $\epsilon$ is the bilayer's dielectric constant, $A$ is the bilayer surface area, and $d$ is the bilayer thickness. Under pressure, just like a normal membrane, the bilayer will bulge into a curved shape. Curvature increases surface area and thus also capacitance, according to equation 1.1. The capacitance change is insensitive to the direction of curvature, so for planar membranes, it occurs at twice the stimulus frequency (see Figure 1.6A). For sufficiently bent bilayers, the frequencies are equal (see Figure 1.6B) [55].

If the membrane is interrogated by a voltage, then the capacitance changes manifest themselves as currents. Although a more rigorous mathematical treatment will be discussed in Chapter 3 , there are some interesting qualitative features of mechanocapacitance that should be noted. Since current is dependent on the rate of change of capacitance, current output occurs at a 90 degree phase difference from membrane deflection. In addition, current output is proportional to voltage, stimulus frequency, initial capacitance of the membrane [36]. It is also inversely proportional to bilayer thickness, as can be seen from equation 1.1. In fact, Ochs saw current output increase more than 200 times when membranes were thinned to black films [55].

The electrowetting effect describes the increase in surface tension of an aqueous droplet in contact with an electrolyte due to an applied potential difference. The effect is not exclusive 

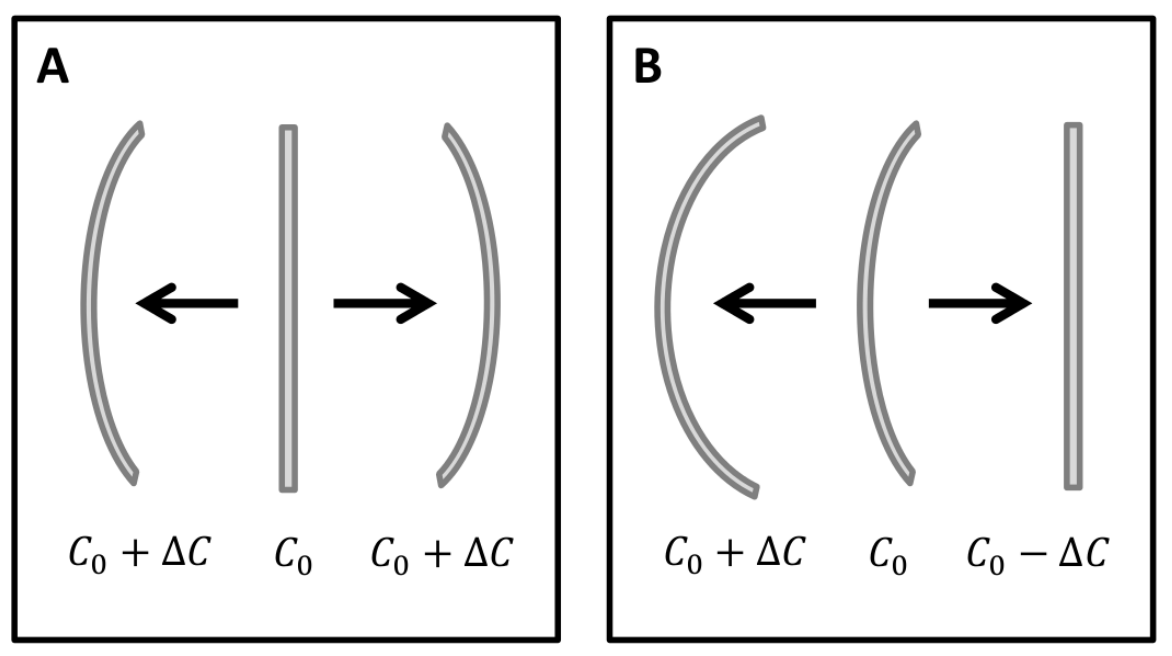

Figure 1.6: Two variations of mechanocapacitance. A) Capacitance change due to membrane bending around a planar configuration. Capacitance increases in both directions. B) Capacitance change due to membrane bending around a pre-bent configuration. Capacitance increases with increased curvature and decreases as membrane becomes planar.

to bilayers, yet it is a significant factor in their mechanical behavior while under an applied voltage. In the case of a DIB, an electric field creates forces that cause the droplet contact angle to shrink. This is accompanied by an increase in the droplet contact area, and hence an increase in bilayer capacitance. This effect has been used by Punnamaraju et al. to control bilayer dimensions. Droplet size and support geometry, however, can limit the amount of area growth and cause saturation. Unbounded electrowetting area growth is proportional to the square of voltage and occurs at twice the input frequency [56].

Flexoelectricity is a result of the asymmetric redistribution of lipid molecules when a membrane is bent. It has been extensively studied by Petrov using pressure-actuated aperture bilayers. The phenomenon is limited to charged lipids, which polarize the bilayer membrane when it is flexed. Pretrov has attributed the behavior to three factors: 1) charge redistribution due to the expansion of the outer monolayer and compression of the inner monolayer, 2) asymmetry of the lipid dipole orientations, and 3) the splayed uniaxial orientation of quadrupoles. The flexoelectric effect occurs at the same frequency as the oscillating pressure with a 90 degree phase difference. However, the exact manifestation of the aforementioned factors depends on whether the driving frequency occurs above or below the lipid exchange rate. This rate governs the insertion of new lipids from the bulk aqueous phase into the 
bilayer. For low oscillation frequencies $(<300 \mathrm{~Hz})$, lipid insertion can significantly change the bilayer polarization. The phenomenon is quite complex, however, it most noticeable at high potentials $(>100 \mathrm{mV})$ and large membrane deflections [57, 54].

Mechanoconductance is the process with the most biological applications and one of the most difficult to replicate experimentally. It does not occur in unmodified bilayers. Rather, it requires embedded mechanosensitive protein channels that change conductance levels based on membrane tension, a phenomenon called "gating." Mechanosensory channels are found all around nature, helping cells to regulate volume, blood pressure and sense contact. It is these channels that are responsible for the electrical response of hair cells [58]. The exact mechanism by which mechanosensory proteins respond to membrane tension is not yet fully understood, but experimental observations have shown tha lipids play a role in transmitting mechanical force [59, 5].

\subsection{Bio-Inspired Principles}

Millions of years of evolution have produced highly effective, specialized biological systems. Hair cells in particular are excellent examples of a smart materials system, with lipid bilayer membranes as the underlying smart material. Smart materials couple different physical domains, and smart material systems apply these to a particular function [60]. The cell membranes of hair cells, through embedded mechanosensory channels, convert mechanical strain to electrochemical impulses. The coupling of hairs or hair bundles to this "smart" strain sensor allows fluid flow measurements of high resolution. However, this system is not easy to replicate in a laboratory: a biological hair cell is small, quite complex, and its biological components are not easily understood nor handled. From the perspective of developing an artificial hair cell, we should not attempt to imitate the whole. Instead, as with most cases of bio-inspired design, we should seek first to identify fundamental principles that are essential to the functionality of the biological system. Here, we present the merits of three key design principles gleaned from the study of hair cells and lipid bilayers, as they might apply to flow sensing. 


\subsubsection{Cilia as flow measurement elements}

The first design principle comes from defining characteristic of hair cells, that is, the use of small cilia (i.e. hairs), or bundles of them, as the mechanical flow-sensing element. One advantage of cilia is that their small size makes them light and unobtrusive to the larger system (e.g. the animal) and does not interfere significantly with the local dynamics of the environment. They are also thin, and thus can sample very narrow regions of a flow field with good sensitivity.

Another advantage is that the cilial structure is simple and adaptable. Two principal parameters of a cilium can heavily influence its mechanical response: base stiffness and length [17]. These can be changed through physical and material properties, as well as by bundling or coupling multiple cilia. Some coupling methods in nature, mentioned in section 1.1, are tip links and cupolas. Through strategic parametric adjustments, cilia can be adapted to

respond to any fluid environment (air, water, etc.). Parameters can even be fine tuned to resonate at specific frequencies, and thus improve sensitivity at that band.

A final advantage is that cilia geometries can be made non-symmetric, providing additional capacity for directional sensitivity. Vertebrate hair cells employ this concept by grading the stereocilia lengths along one axis of the bundle [12]. The result is increased sensitivity to flow along the same axis. However, even a single cilia can be made non-symmetric to change its directional sensitivity properties [53].

\subsubsection{Array sensing}

The modularity of hair sensors facilitates another type of biological design principle: sensory arrays. These are defined as groups of sensors distributed in a precise geometric pattern that map information both in space and time. In essence, the relative outputs of sensors at different location help to track the changes in a two or three dimensional stimulus field over time. The biological systems studied in section 1.1 featured arrays with up to thousands of hair cells. All of these arrays were organized primarily into one-dimensional groups: A trichobothria cluster [10], a single line on the basilar membrane [3] and a lateral line [27]. However, multiple instances of these groups are distributed throughout animals' bodies, ultimately helping to resolve more complex dimensional information. The presence of two ears (i.e. two cochleae), for example, helps mammals localize noise sources based on the relative 
delay of one ear to the other [6]. Through this and similar examples, natures suggests that simple arrays organized strategically may be more effective than dense, multi-dimensional ones.

What is not yet clear is how most animals process the vast amount of information from large hair cell arrays so quickly and effectively. Because cilia do not significantly alter the local flow, it is possible to process every sensor signal individually before synthesis. However, such a method would require an enormous amount of processing, for animal brains as well as for computers. Instead, animals are seen to employ mechanical filters to simplify incoming information. The basilar membrane, for example, performs a type of spacial Fourier analysis [25]; fish canals act as fluid velocity high pass filters [16]; and trichobothria lengths are tuned to specific spectral bands [10]. It has been also shown that nerve fibers from fish hair cells collect into discrete nerve bundles [16], suggesting that outputs are combined before reaching the brain in order to simplify processing.

\subsubsection{Biomolecular Transduction}

A cilium, of course, needs a strain sensor at its base to produce a fully functional hair cell unit. Biological hair cells sense strain through mechanosensory proteins that respond to tension in the cell membrane. Incorporating these proteins into synthesized bilayer membranes is the most promising way to harness them for engineering applications. However, this has been severely limited by current scientific understanding of the precise functional mechanics of mechanoreception at the cellular level. Nevertheless, bilayers, which are better understood, have demonstrated other biological mechanoelectrical transduction properties: mechanocapacitance and flexoelectricity. These have already been explored within hair cell structures [61]. In addition, the DIB method is capable of being adapted to form large bilayer networks [51]. These qualities make bilayers a promising platform on which to build arrays of artificial hair cells completely from biological materials.

\subsection{Bio-inspired Artificial Hair Cells}

The astounding variety of biological applications for a sensor based on a relatively constant operational principles has inspired recent developments in the field of artificial hair cells, or 
AHCs [62, 20]. AHCs seek to mimic some or all of the useful engineering properties of their biological counterparts, outlined in the previous section, to measure fluid movement, whether by flow or acoustic pressure waves. The examples presented here will focus on flow, since the ultimate research goal is airflow measurement. We divide AHCs into two categories based on their principal transduction mechanism. MEMS based AHCs are those that measure cilial deflection via conventional silicon based components, such as capacitors or piezoelectrics. Bilayer based AHCs instead use the transduction properties of organic lipid membranes.

\subsubsection{MEMS based AHCs}

Recent interest in the sensing abilities of biological hair cells has given rise to developmental research on artificial hair cell sensors for practical applications. The most common design principles employed by these sensors are high aspect ratio cilia as sensing elements, directional sensitivity and modularity. In order to operate at comparative scales but maintain cost efficiency, most are fabricated using established MEMS assembly techniques. Current designs are based on a variety of MEMS sensing technologies, including piezoresistive [62], capacitive [63], thermal [64], magnetic [65], piezoelectric [66], and optical [67]. Of these AHCs, those based on piezoresitance and capacitance are the most mature, and thus will be covered in more detail.

Liu's group at Northwestern University has already developed several generations of piezoresistance based artificial hair cells for flow applications. The sensors ranged from 500 to 1100 $\mu \mathrm{m}$ in cilium length and could be adapted to both air and water flow [62, 20]. The general sensor design utilized a stiff vertical hair supported by a horizontally cantilevered bar, made from piezoresistive material. Induced drag from fluid flow pushes on the hair and the resulting bending on the bar can be observed as a change in resistance [68, 69]. Because torsion of the piezoresitor bar does not produce appreciable changes in resitance, the sensor exhibits very clear bi-directional sensitivity. The AHC design evolved from a flat, thin cilia [62] to cylindrical ones [70] to improve the angular resolution of the device. The team also moved from silicon to polyurethane as the hair material for improved robustness in high flow conditions [68, 69]. Peleshako et al., directly inspired by the fish neuromast, greatly improved sensitivity and dynamic range in water by encapsulating the hairs in a hydrogel "cupula" [71. The group hypothesized that the artificial cupola maximized drag forces through a larger cross-section and increased viscous friction between the gel and the fluid. The best 
sensitivities achieved by this generation of sensors were in water: less than $1 \mathrm{~mm} / \mathrm{s}$ for a standard cilium and $0.075 \mathrm{~mm} / \mathrm{s}$ for the cupola-encapsulated design [20, 70, 71]. The AHC sizes and sensitivity values place them well within the range of spider trichobothria. One notable discrepancy, however, is that the AHC sensitivity values are for water, not air, which is an easier medium to measure.

Another group at University of Twente used a MEMS variable capacitor to transduce hair motions. A flexibly-supported plate with electrodes at opposite ends was suspended above a conductive base. A polyurethane cilium is rigidly connected to the plate. Viscous torque on the cilium bends the plate in the direction of either electrode, producing changes in capacitance between the base and electrodes [20, 63]. Both spiral and torsional supports for the flexible plate are tested; results indicate that the torsional support provided greater stiffness for translation than for rotation making it a preferred option [72]. Strategic placement of the electrodes allowed for directional sensitivity which was experimentally determined to have an 8-like shape. Various iterations of the basic sensor design have been tested, achieving sensitivities to air flow as low as $1 \mathrm{~mm} / \mathrm{s}$ [20]. In addition, the researchers were able to fabricate hundreds of hair cell units into rectangular arrays [63, 73]. The arrays, however, were mostly used to increase overall capacitance (and thus sensitivity) by connecting units in parallel. The researchers are still investigating techniques for individual unit interrogation and how to generate spatial data [74].

Additional classes of AHC's demonstrating different transduction principles are still relatively new [20]. Some examples include: the use of phototransitors to detect bending in optical fibers [67]; PZT fibers which are senstive to bending, thus combining the cilium and transducer [66]; and units based on hot wire anemometry, whose thermal operation is markedly different in principle from deflection-based sensing [64, 75].

Overall, state of the art MEMS based AHC technology has demonstrated comparable size and sensitivity to some hair cells in nature. However, relatively little research has yet ventured into spacial analysis of flow fields using arrays. Chen et al. are one of the few that have attempted this task, but only for known, well defined sources. Using lateral line inspired configuration, they were able to locate disturbance sources; however, their location algorithms depended on an a priori model of the disturbance field. Furthermore, the artificial lateral line was based on hot wire anemometry and not cilia deflection [64, 75]. The obstacles to further AHC development can be summarized in three challenges as in [20]. The first is that improving $\mathrm{AHC}$ performance requires simultaneous optimization of two interactions: the 
structure-fluid and the structure-transducer interaction. The former can be quite difficult in all but the most simple flow scenarios. Secondly, effective methods for large array information processing must be developed for flow mapping, object localization, and tracking. This is hindered by the fact that neural decoding of hair cell information in vertebrates is not yet fully understood. Finally, concerns exist about whether flow interference among sensors could limit array sensing applications. Studies have shown that interactive coupling occurs between trichobothria hairs, although it is though to be insignificant [76]. Nevertheless, the considerable geometric differences of AHCs may make them more susceptible to this phenomenon.

\subsubsection{Bilayer Based AHCs}

Within the last four years, some researchers have sought to add another level of bio-inspiration to AHCs by using biomolecular transduction principles, instead of silicon components. The goal of these sensors is not to compete with their MEMS counterparts, but rather to explore the engineering potential of biological materials. Pioneering work by Sarles and Leo showed that lipid bilayers, the foundation of the cell membrane, can be used as mechanoelectrical transducers [77, 61]. By virtue of the flexoelectric effect, bending of the bilayer membrane due to hair motion induces a change in capacitance that can be measured as ionic current flow across the membrane [55, 57]. The fabrication method for these sensors requires that the bilayer be assembled in situ while submerged in an oil solution. The first generation of these membrane-based AHCs were assembled in polyurethane substrates via the regulated attachment method or RAM [52, 78]. A hydrogel "follicle" served as both a rigid support for the hair and as a hydrophilic scaffold on which to form the lipid bilayer. Subsequent designs have subjected the sensor to various types of excitation: continuous air and water flow [61, 79], impact hammer [80], mechanical perturbation [81, 82] and oscillating fluid flow [83]. These studies validated the functionality of membrane-based AHCs in various conditions, yet a detailed understanding of their dynamic behavior has proved challenging largely due to high variability in bilayer, hair and hydrogel properties. Often, low sensor output and inability to measure input excitation can lead to poor characterization coherence.

Furthermore, while networks of multiple lipid bilayers have been demonstrated [84, 51, 85], little has been done with arrays of membrane-based AHCs, especially with respect to flow analysis. One reason is that the RAM technique, the basis of assembly for most generations 
of AHCs, requires mechanical compression of a large supporting substrate to create bilayers [78. This feature poses a design challenge when attempting to create compact arrays of these structures. However, a 2012 study by Bayley showed that durable bilayers can be formed between lipid-coated, solid hydrogel shapes suspended in oil[51]. The author was the first to apply this concept to a membrane-based AHC, which was supported entirely by hydrogels themselves [53]. This allowed for much greater flexibility in hair cell orientation and modularity in substrate design, advancing the possibilities for creating a functional AHC array.

\subsection{Document Overview}

This paper presents a novel bilayer based AHC platform, developed for array implementation by applying some of the core design principles of biological hair cells. It builds on the bio-inspired research presented in the previous section, especially membrane-based AHCs. The sensor design expands on this class of sensors by fully supporting lipid bilayers on movable hydrogel scaffolds. It also provides the modularity needed to build linear AHC arrays. First, the document outlines key design, fabrication and material considerations toward improved sensor performance, namely signal to noise ratio, airflow sensitivity and bilayer durability. Preliminary tests show bilayer stability of approximately 30 minutes and an order of magnitude improvement in current output over previous designs. Second, we discuss the basic theory necessary to understanding hair mechanics and the biological basis for the lipid bilayer's transduction properties. Third, a detailed characterization of a single hair cell system's response to base excitation and short air pulses demonstrates the output characteristics of the AHC. This information leads to inferences about the coupling relationship between hair and bilayer membrane. Fourth, a spectral analysis of the AHC system under varying voltages and air flow magnitudes helps to build simple, predictive models for the sensitivity properties of the AHC. And finally, based on these results, we implement an array sensing strategy that involves mapping frequency content to stimulus location by "tuning" individual hairs. 


\subsection{Contributions}

The following key contributions from this research advance upon previously demonstrated capabilities of lipid bilayers in a sensory context:

- Development of an artificial hair cell platform based on fully hydrogel supported lipid bilayer membranes. This design had two distinct advantages. First, bilayers could be manipulated and oriented freely via the hydrogels, without the need for a contacting substrate. The flexibility in layout facilitated the creation of compact, modular units. Second, hydrogel size was adjusted to accommodate stiffer hairs, which led to improved signal to noise ratios. Nanoamp range output currents were consistently observed, an order of magnitude higher than any other recently developed membrane-based sensors. Overall, the AHC unit is an useful and versatile platform for studying bilayer membrane response to many stimuli (water flow, touch, etc.).

- Spectral characterization methods of an AHC response in airflow. These methods were able to calculate a clear input-output relationship for a delicate and complex dynamic system, which was difficult to interrogate by traditional modal analysis techniques. The methodology was used to understand bilayer behavior, but could be easily expanded to the study of small hair cells in general.

- Array implementation of independently controlled bilayer-based artificial hair cells. This includes the development of a novel method of frequency to location mapping whose strategy involved using hairs of different lengths. This is the first demonstration of using an array of this type of sensor for stimulus localization. 


\section{Chapter 2}

\section{Design and Testing Methods for a Novel Artificial Hair Cell Array}

This chapter builds upon earlier generations of research by Sarles and Leo [77, 61] to create a membrane-based AHC which is supported entirely by hydrogels. This allows for much greater flexibility in substrate design, bilayer manipulation and hair cell orientation, ultimately enabling the creation of closely-spaced AHC arrays. The section begins with a description of the functional design of the AHC "unit" and then explains how these units are combined to form AHC arrays.

\subsection{The Artificial Hair Cell (AHC)}

\subsubsection{Components of the AHC Bilayer Membrane}

This study utilized droplet interface membranes (DIBs) formed on hydrogel supports through a hybrid of two methods developed by Bayley et al.: lipid-in DIB's [86] and lipid coated hydrogels [51]. The aqueous solution used to form lipid-in DIB's consists of $2 \mathrm{mg} / \mathrm{mL}$ 1,2 diphytanoyl-sn-glycero-3-phosphocholine (DPhPC, Avanti Polar Lipids, Inc.) phospholipids suspended in $10 \mathrm{~mL}$ of $500 \mathrm{mM}$ potassium chloride (KCl, Sigma Aldrich), $10 \mathrm{mM}$ 4-morpholinepropanesulfonic acid (MOPS, Sigma Aldrich), pH 7 ionic buffer solution. The preparation of this solution is described elsewhere [86]. The hydrogels are made from a 
Rodrigo Sarlo Ch 2: Design and Testing Methods for a Novel Artificial Hair Cell Array 28

mixture of $8 \mathrm{mg}$ polyethylene glycol dimethacrylate (PEG-DMA, MW $=1000 \mathrm{~g} / \mathrm{mol}$, PolySciences, Inc.), $0.05 \mathrm{~g}$ Irgacure 2959 photoinitiator (Ciba) dissolved in $10 \mathrm{~mL}$ of the same ionic buffer solution as the phospholipids $(\sim 40 \% \mathrm{w} / \mathrm{v})$. When cured via UV light, the hydrogels photopolymerize, providing a rigid yet hydrophilic environment that serves to anchor the aqueous lipid solution. This facilitates the manipulation of aqueous droplets. Each hydrogel is coated with a $\sim 0.2 \mu \mathrm{L}$ lens of the aqueous lipid solution and submerged in hexadecane oil (99\%, Sigma). The hydrophilic-hydrophobic interface at the hydrogel-oil boundary creates the necessary conditions for lipid monolayer self assembly, typically in 5-10 mins. At this time, the surfaces of the two lenses can be brought into contact to initiate bilayer growth.

\subsubsection{Unit Fabrication}

The artificial hair cell "unit" is defined as one hair-membrane system that acts as a single sensor. The primary design goal for these units was that they be modular, capable of being assembled into tightly-packed arrays while maintaining individual control of each system. Lipid bilayer membranes are delicate systems and can vary greatly in formation and durability, making bulk manipulation of the membranes not a feasible option for this application. Therefore, independent control was critical to maintain bilayer stability throughout experiments lasting between 30-60 minutes. Through careful material selection, refined mechanics and fabrication methods, the design was also aimed at improving the sensor's durability, repeatability and signal to noise output.

The hair cell sensor unit consists of two main components: 1) a mechanical sensing element, i.e. the "hair", which deflects or vibrates when excited by a fluid flow, and 2) a transduction element, the bilayer membrane, which converts the mechanical vibrations to an electrical signal. A basic diagram of the device is shown in Fig 2.1A. The unit was housed in a recessed chamber to hold the oil, with openings on either side for $1 \mathrm{~mm}$ diameter glass pipette tubes (1B100-4, WPI, Inc.). The tube on the left of the figure held a "base hydrogel" which provided a rigid, hydrophilic environment to anchor the aqueous lipid solution and also to support the hair. The smaller "secondary hydrogel" protrudes slightly from the right pipette and anchors a second lipid lens. This pipette is attached to a micromanipulator (SM325, WPI, Inc.) that allows for the precise control of the hydrogel's horizontal position. Silver-silver chloride $(\mathrm{Ag} / \mathrm{AgCl})$ electrodes, used for all electrical measurements, run along the length each pipette and insert into the hydrogels. Note that the base hydrogel is always 
connected to ground whiled the secondary hydrogel secures the positive electrode.
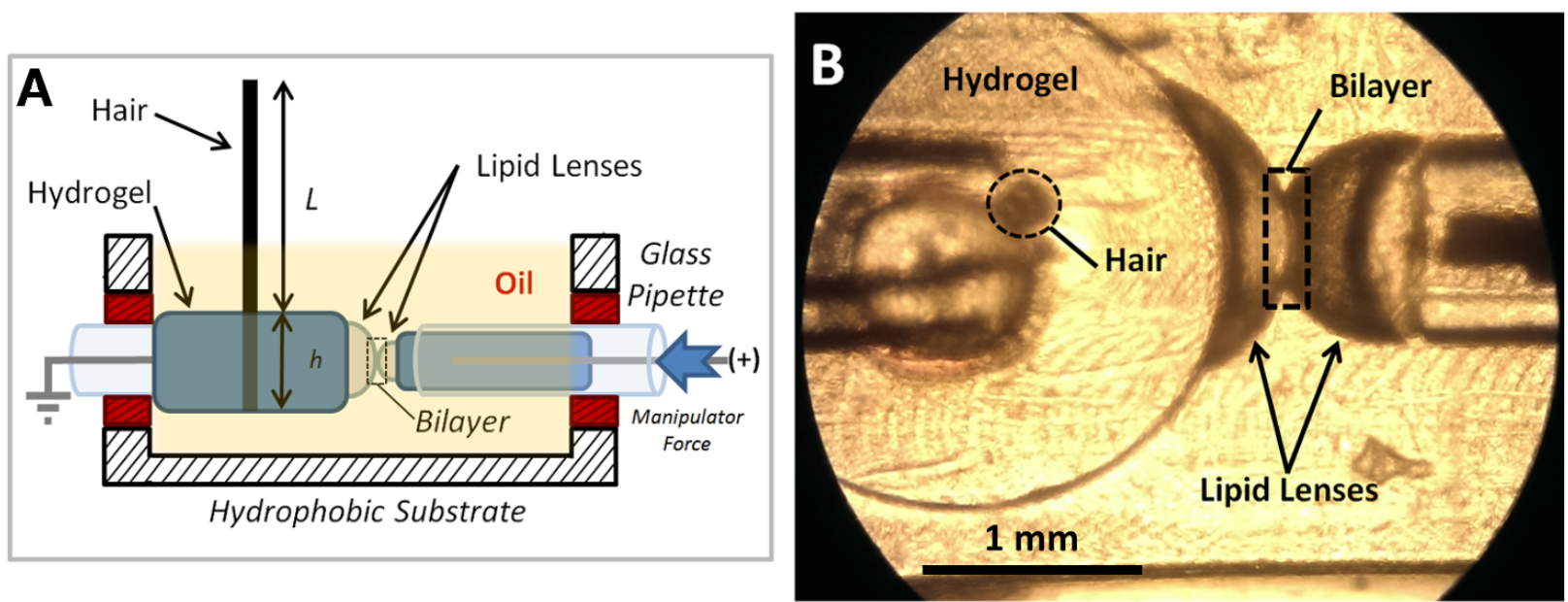

Figure 2.1: A) A cross-sectional view of substrate chamber housing the hair cell unit shows how solid hydrogels serve to support the hair as well as the lipid lenses that are brought into contact to form a bilayer. The hydrogels were cured onto glass pipettes containing electrodes through which all electrical measurements were made. $L$ indicates the free length of the hair while $h$ indicates the height of the hydrogel base. B) A microscope image taken from underneath the substrate illustrates a typical bilayer formation.

The substrate chamber measured $12 \times 3 \times 4 \mathrm{~mm}$ and was machined from transparent acrylic (.22 in, OPTIX), enabling microscope viewing from below. Acrylic is a hydrophobic material, chosen to reduce the propensity of the aqueous solution to adhere to the substrate walls. The hairs were fabricated from $1 \mathrm{~mm}$ diameter solid glass rods (GR100-4, WPI, Inc.). To create the hairs, the glass rods were heated and stretched until very thin, then cut to the desired length. The result was a slightly tapered hair with base diameter of about $300 \mu \mathrm{m}$ and tip diameter of about $100 \mu \mathrm{m}$. The manufacturer provides the glass modulus of elasticity $(E)$ and density $(\rho)$ as $71.6 \mathrm{GPa}$ and $2333 \mathrm{~kg} / \mathrm{m}^{3}$, respectively. The original hairs are cut by hand to the appropriate lengths.

Assembly of the complete hair cell unit requires careful fabrication and detailed methodology. First, the base hydrogel is injected into a polyurethane mold $(2.5 \mathrm{~mm}$ dia $\mathrm{x} 2 \mathrm{~mm}$ ) which also holds the electrode, pipette (1B100-4, WPI, Inc.) and hair in their respective positions. The gel is then UV cured until solid enough to hold the pipette and hair on its own. The secondary hydrogel is simply suctioned into the glass pipette and cured with an electrode held in place. Gel curing takes approximately 2 mins at a distance of $3 \mathrm{~mm}$. When fully cured, the hydrogels assemblies are inserted into either end of the substrate chamber by 
threading the pipettes through rubber gaskets, which seal the chamber and support the gel assemblies in mid-air. The chamber then is filled with hexadecane oil (99\%, Sigma Aldrich), submerging the hydrogels. Using a micropipettor, each is coated with a $0.2 \mu \mathrm{L}$ lens of the aqueous lipid solution. The lipid solution is left intact for 5-10 minutes to allow for monolayer self assembly. After this time, the right monolayer is moved into contact via the micromanipulator to initiate bilayer formation. Fig. 2.1 $\mathrm{B}$ shows a microscopic image of a successful bilayer assembly. Bilayers can take 1-10 mins to form and stabilize. The bilayer size can regulated by adjusting the horizontal position of the secondary hydrogel.

\subsubsection{Array Assembly}

The great complexity of biological hair cells comes from their ability to work together large arrays, such as in the lateral lines in fish [11, 14, 15]. While some groups have assembled large arrays of traditional AHC's, previous generations of membrane-based AHC's have been limited to single units. One of the ultimate goals of this study was to present a proof of concept of a functional membrane-based AHC array. Here the author describes the methodology for assembling these arrays and measuring their output based on current equipment limitations.

The AHC unit design allows for modularity in one dimension, yet critical choices still needed to be made regarding the array size, unit spacing, and hair lengths. Creating large $(>50)$, durable arrays of DIB's (without hairs) is still an active area of research, so it was impractical to attempt such scales for AHC's. Considering this and the lack of MEMS scale fabrication equipment, this study limited itself to arrays of three hair cells. The units were machined side-by-side into a single $0.22 \mathrm{in}$. acrylic sheet with a spacing of $7 \mathrm{~mm}$ between the hairs (Figure 2.2. . This was compact enough to allow the air source to excite multiple hairs at once while being separated enough for the manipulation of the pipettes. The entire platform was movable by hand to allow the viewing of different AHC compartments.

The nature of the array sensing strategy (described in Ch. 6) required that hairs be of different lengths so that each AHC's output could be sufficiently distinguished. In addition, there were lower and upper limits on the length of the hairs due to fabrication. Any hairs longer than $50 \mathrm{~mm}$ tapered to a very thin diameter and lost rigidity. Any hairs shorter than $15 \mathrm{~mm}$ were excessively stiff and did not protrude enough from the substrate to be sufficiently excited by the air source. The hairs were trimmed to lengths of approximately 40, 30, and 20 


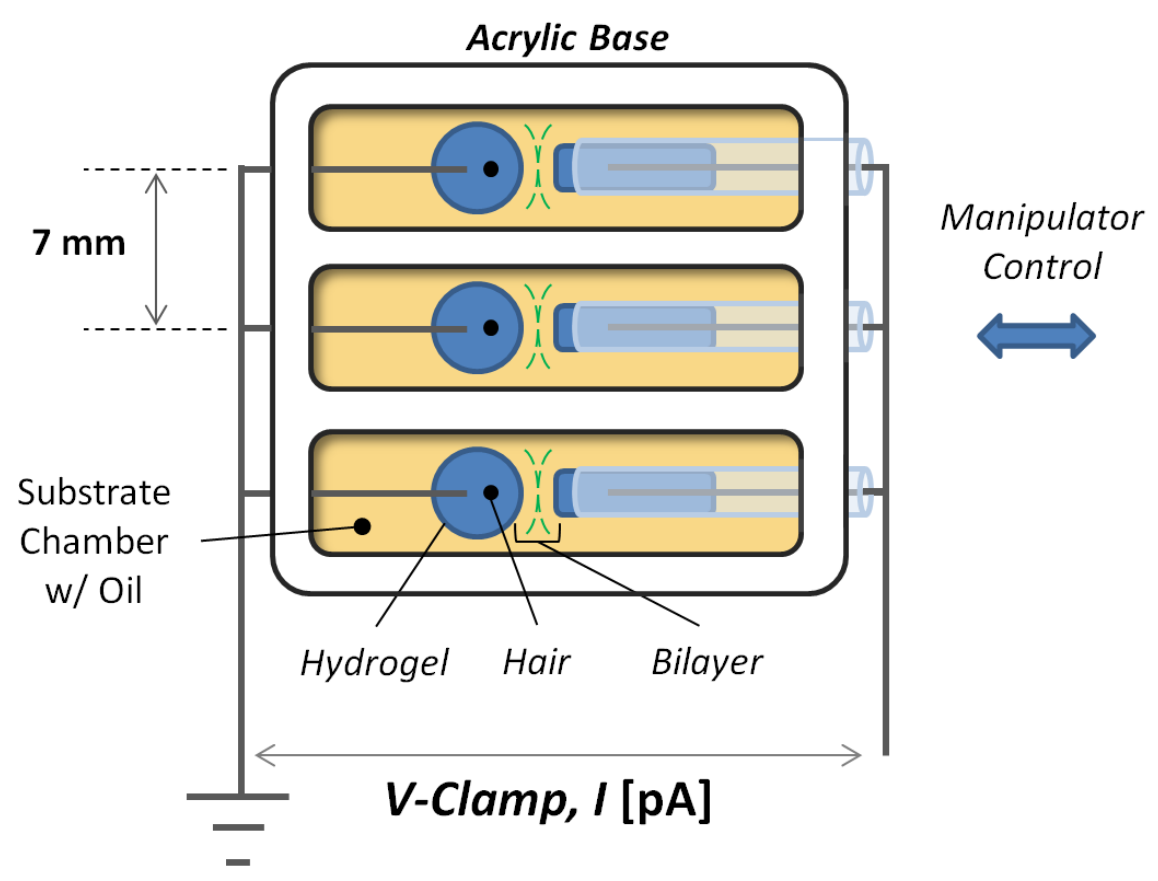

Figure 2.2: A top view diagram depicting how AHC units were arranged into an array. The substrate chambers were machined into a single piece of acrylic with a spacing between hairs of approximately $7 \mathrm{~mm}$. The electrodes were wired so that each bilayer was connected in parallel, essentially forming a larger capacitor. The circuit was voltage clamped and the current output of the system was measured

$\mathrm{mm}$ to fit within these constraints. They were arranged in descending order. Some studies have suggested that bilayer membranes may have a frequency-dependent sensitivity, which could mean different sensitivities to different hair lengths [52, 87]. However, this was not taken into account when selecting hair lengths.

\subsubsection{Design Evaluation}

The fabrication method outlined above was chosen to increase current response, improve sensor durability, and limit noise. The use of a glass hair had numerous advantages over the polymer fiber hairs of previous sensor generations [53, 61]. Firstly, glass hairs are much more uniform in mechanical properties (e.g. density, elastic modulus) and geometry, making them easier to model. Secondly, their higher stiffness couples more effectively with the hydrogel mass, providing better transfer of energy to the bilayer membrane. A fiber hair can deflect 
large amounts without transferring much motion to the hydrogel. One disadvantage is that glass hair are brittle and can break during fabrication.

The base hydrogel chemical composition $(\sim 40 \% \mathrm{w} / \mathrm{v})$ and size $(h=2 \mathrm{~mm})$ were selected to provide a solid support for the hair during excitation while still being able to effectively transfer vibrations to the membrane. It was observed that larger hydrogels $(h=4 \mathrm{~mm})$ were more robust and easier to model but could attenuate the oscillations felt by the membrane by more than an order of magnitude. Smaller gels $(h=1.5 \mathrm{~mm})$ provided better vibration transfer to the membrane but detached too easily from the pipette. The rounded shape of the hydrogels (see Fig. 2.1B) and their suspension in oil helped to facilitate formation of the lipid lenses. Flat surfaces and/or contact with the substrate walls often resulted in migration of the lipid droplets during testing.

Finally, the use of pipettes helped to insulate the electrodes and reduce electrical noise contributions. Care was taken to leave minimal amounts electrode length exposed. In addition, because mechanical vibrations of the positive electrode have been shown to be a source of current [82], the positive electrode was always inserted into the secondary hydrogel (see Fig. 2.1A). Typical ambient noise measurements were about 0.1-1 pA RMS. Additional control tests confirmed that vibrations from adjacent hair cells ( $5 \mathrm{~mm}$ apart), did not contribute to significantly to the measured current signal.

\section{$2.2 \quad$ Experimental Measurements}

The measurement of picoamp-scale currents produced by the bilayer membranes within the AHC units required suitable electrodes and a low-noise environment. Silver/silver chloride $(\mathrm{Ag} / \mathrm{AgCl})$ were chosen because they allow the free and reversible transport of ions from the buffer solution, resulting in little added impedance to the electrical circuit [88, 89]. They are made by chloriding $125 \mu \mathrm{m}$ silver wire (Goodfellow) in household bleach for several hours. The testing was carried out on a Axiovert 40CFL inverted microscope (Carl Zeiss) so that the hydrogel positions could be observed through the bottom of the transparent acrylic substrate. A 3-axis micromanipulator (SM325, WPI Inc.) was used to control the position of the hydrogels via the glass pipettes. The entire workstation was shielded by a Faraday cage which was grounded to an earth ground connection as well as to the microscope, micromanipulator, and electrical measurement devices. A picture of the test workstation is 
shown in Figure 2.3 .
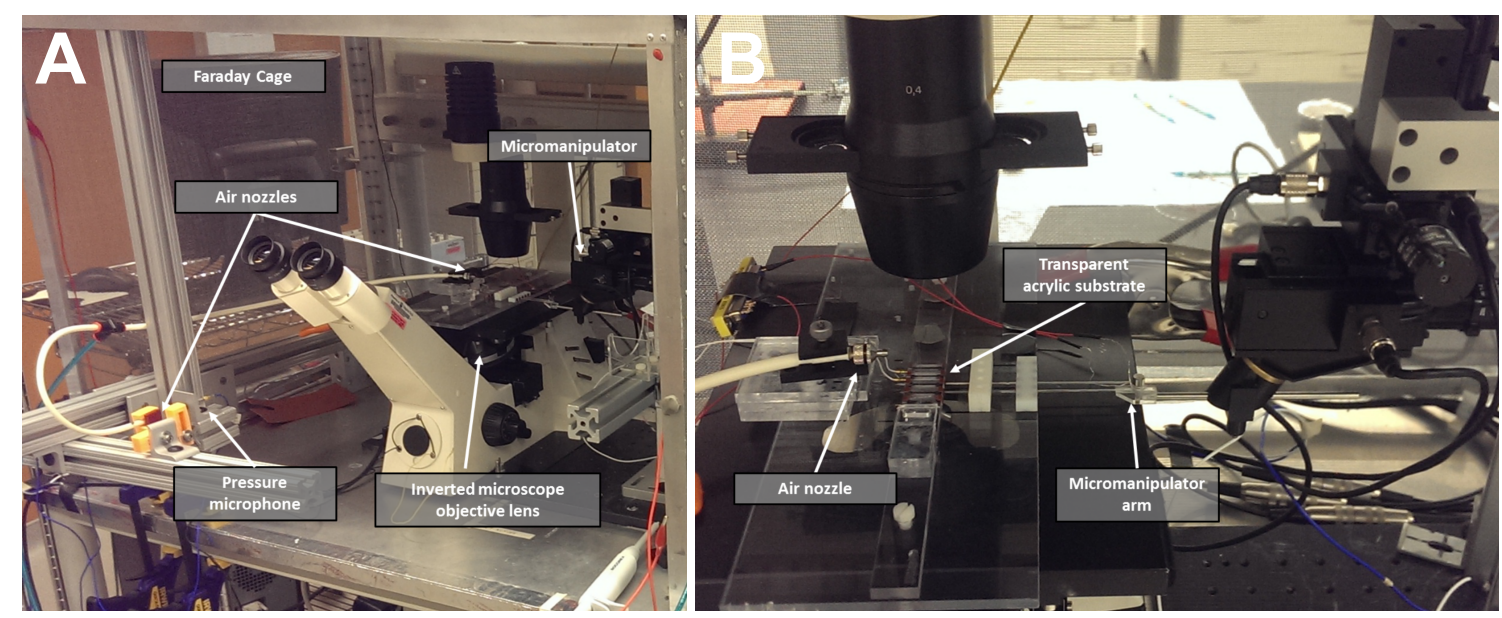

Figure 2.3: A) Low-noise workstation with relevant equipment and Faraday cage shielding. B) Close up image on the microscope table showing the positioning of the micromanipulator and air nozzle relative to the test $\mathrm{AHC}$ substrate

All electrode measurements were performed through an AxoPatch 200B (Molecular Devices) in a voltage clamp configuration, whereby negative feedback circuit measures the amount of current necessary to hold the membrane at a command voltage [90]. An offset voltage was applied to membrane to cancel any existing potentials between electrodes. In this way, a command voltage of zero volts would correspond to a current output of zero amps. The measured currents were low-pass filtered at $2 \mathrm{kHz}$, then sampled at $5 \mathrm{kHz}$ by a DigiData 1440A (Molecular Devices). Bilayer electrical properties are interrogated electrically by applying a $10 \mathrm{~Hz}, 20 \mathrm{mV}$ peak-to-peak triangle wave across the electrodes. Because the membrane current response displays purely capacitive behavior at $10 \mathrm{~Hz}$ [91], it will follow the voltage-current relationship for a simple capacitor:

$$
i(t)=\frac{d}{d t}(C V)=C \frac{d}{d t}(V)+V \frac{d}{d t}(C)
$$

An undisturbed bilayer has a fairly constant capacitance $\left(\frac{d}{d t}(C)=0\right)$, thus the capacitor relation can be simplified to Equation 2.1. A triangle wave voltage produces a square wave current response scaled by the membrane capacitance. The transition from two monolayers in contact to a bilayer, i.e. bilayer formation, is signalled by a large growth in the magnitude of the square wave (Figure 2.4). This reflects the increased capacitance created by the 
new bilayer. After the initial growth and formations, the bilayers will stabilize to constant capacitance and may last for $>30$ mins before breaking down. The capacitance value $(C)$ may easily calculated according to equation 2.1. The area of the membrane may be estimated from a relationship developed for similar lipids of $0.6 \mu \mathrm{F} / \mathrm{cm}^{2}[50]$. The bilayers in this study are on the order of $0.05-0.1 \mathrm{~mm}^{2}$ in area and 0.5-1 $\mathrm{nF}$ capacitance.

$$
i(t)=C \frac{d}{d t}(V)
$$
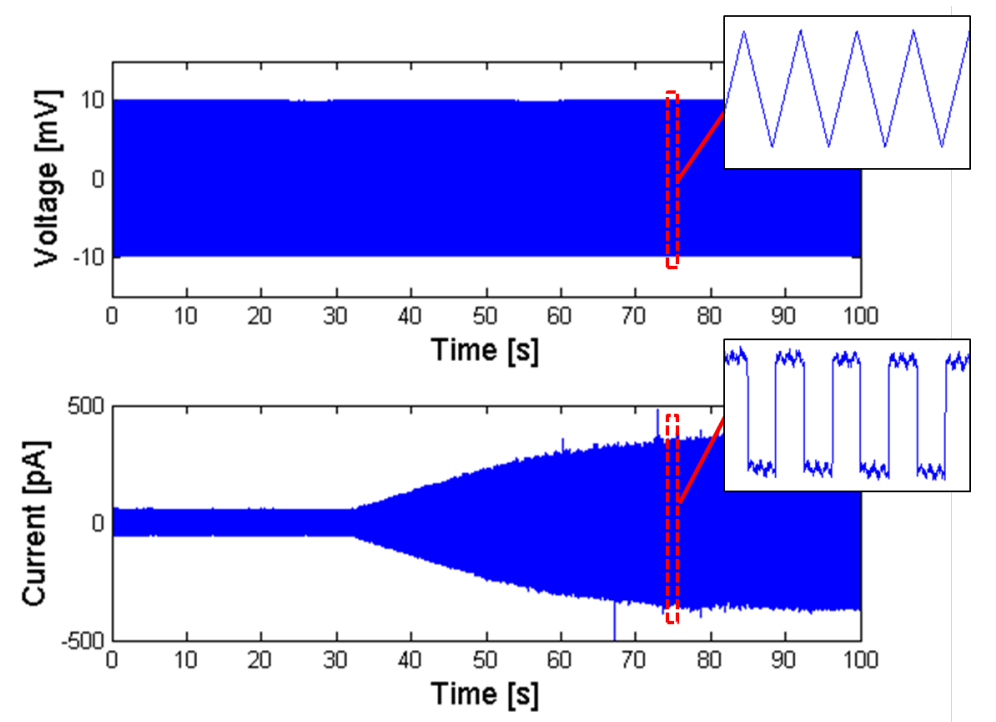

Figure 2.4: Real-time interrogation of the AHC membrane by applying a $10 \mathrm{~Hz}, 20 \mathrm{mV}$ peakto-peak triangle wave (top) and monitoring current output (bottom). The output square wave magnitude is directly proportional to the bilayer capacitance. The sudden increase in the current output at around 35 seconds reflects a capacitance increase and signals bilayer formation and growth.

For array testing, the three AHC units were wired in a parallel configuration to the AxoPatch 200B amplifier's only input channel as shown in Figure 2.2. The circuit was voltage-clamped identically to when measuring a single unit. The measured current was the combined output of the three AHC units. Bilayers were formed one at a time while the circuit was interrogate via a voltage a triangle wave. Successful bilayer formation was indicated by successive increases in current output magnitude as seen in Figure 2.5.

For all hair vibration tests, the membrane was clamped at various constant DC potentials. For the air pulse excitation tests, a 1/4 in. pressure microphone (Y378C10, PCB Piezotronics 


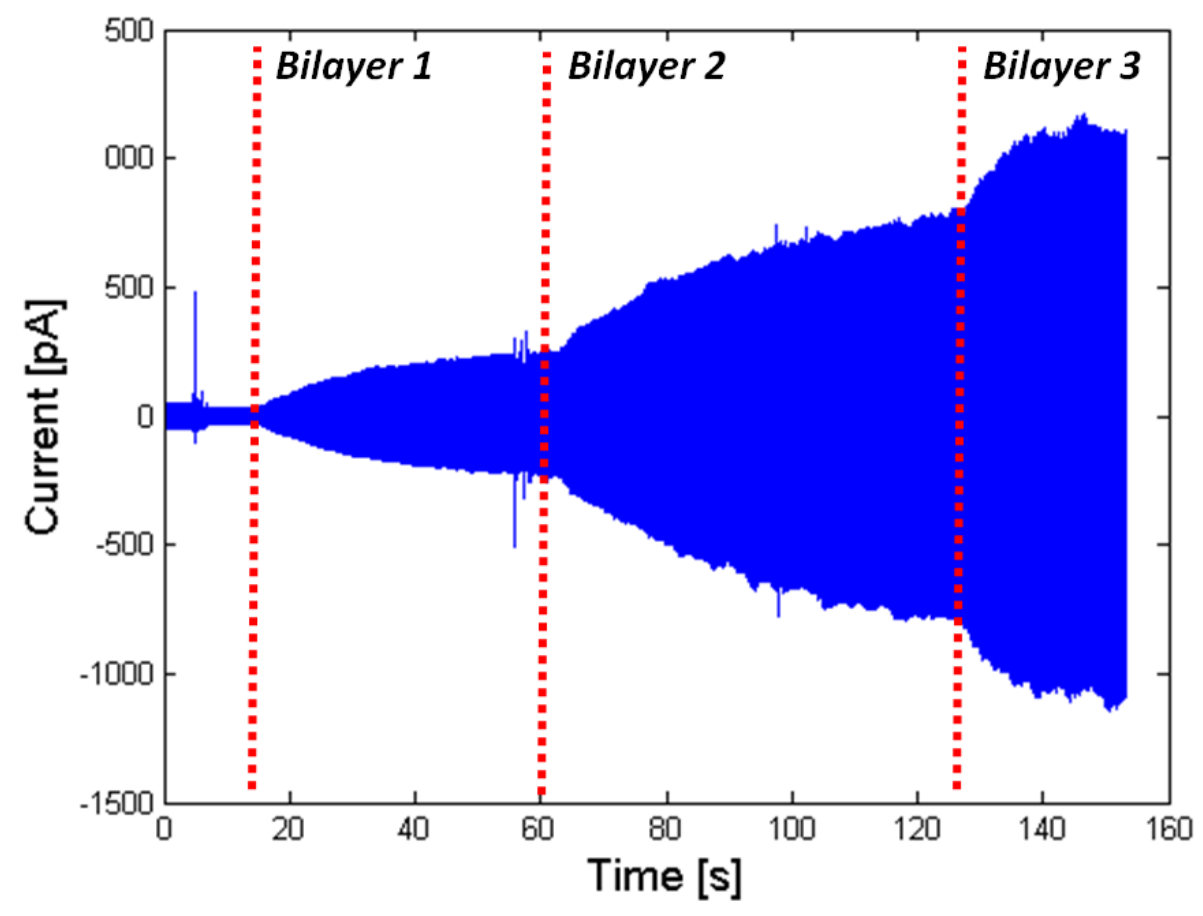

Figure 2.5: A top view of the experimental setup for air pulse excitation shows its relative orientation to the hair cell unit. The air line was split evenly and placed at a distance of $15 \mathrm{~mm}$ from both hair and microphone. The air pulse was aimed at a height $20 \mathrm{~mm}$ above the base of the hair. The air pressure input to the microphone (measured in volts) was compared to the current output (pA) of the voltage clamped hair cell.

Inc.), also show in Figure 2.3, was used to measure the air pulse magnitude. It was connected to the DigiData 1440A via an external signal conditioner (4110C, Dytran). For constant airflow tests, a mass flowmeter (FMA-A2321, Omega Inc.) monitored the airflow magnitude.

\subsection{AHC Testing Methods}

Biological hair cells measure flow magnitude, direction, and frequency of various types of fluids [8, 10, 12, 11]. In this study, we focus on sensing airflows which vary both in magnitude and location. While this can be achieved most effectively through arrays of AHCs, it critically important to first understand the output behavior of individual units. Thus two testing methods, base and air pulse excitation, were dedicated to characterizing the frequency response of single AHC units. The third test, excitation by a constant air flow, was aimed at exploring the effects of excitation magnitude and location of both single units 
Rodrigo Sarlo Ch 2: Design and Testing Methods for a Novel Artificial Hair Cell Array 36

and arrays.

\subsubsection{Base Excitation}

Base excitation was used to determine the vibrational modes of the hair. Since the goal of this method was to analyze purely the hair dynamics, no lipids or oil were used. Rather, a modified substrate allowed the hair to be exposed so its entire length could be scanned. An image of the base excitation test setup is shown in Figure 2.6. The dynamic response of the hair was measured using a PSV-400 scanning laser vibrometer (Polytec) on a 13 point, one-dimensional mesh along the length of the hair and at a distance of $50 \mathrm{~cm}$. The hair was painted white for better reflectivity. A 7-lb modal shaker was use to drive the base of the hair using a $3 \mathrm{~s}$ frequency sweep from $5 \mathrm{~Hz}$ to $2 \mathrm{kHz}$. The responses to three consecutive sweeps were time averaged for each scan point. A load cell (PCB Piezotronics) measured the force applied by the shaker to the base. In order to isolate the hair from environmental vibrations, the shaker and hair holder assembly was placed on an anti-vibration table (SmartTable, Newport). Base excitation was used instead of air excitation in this case because the latter induced off-axis vibrations of the hair which were difficult to measure via laser vibrometer.

\subsubsection{Air Pulse Excitation}

For the sensor characterization portion of the results (Ch. 4), a sharp air pulse was used to resemble an impulse excitation. A single active AHC unit was excited by regulating air flow from a pressurized tank via a digitally controlled valve (T3110, Marsh Bellofram) as seen in Fig. 2.7. One branch of the air line excited the hair in a direction perpendicular to the bilayer. The second branch was used to monitor the direct air input through a pressure microphone (Y378C10, PCB Piezotronics Inc.). To create the pulse, the air was pressurized to 40 psi then released rapidly by opening the valve at $0.5 \mathrm{~ms}$ intervals. The air was fed through $3.2 \mathrm{~mm}$ diameter plastic tubing and a $1 \mathrm{~mm}$ diameter nozzle was used to concentrate the flow of air. 


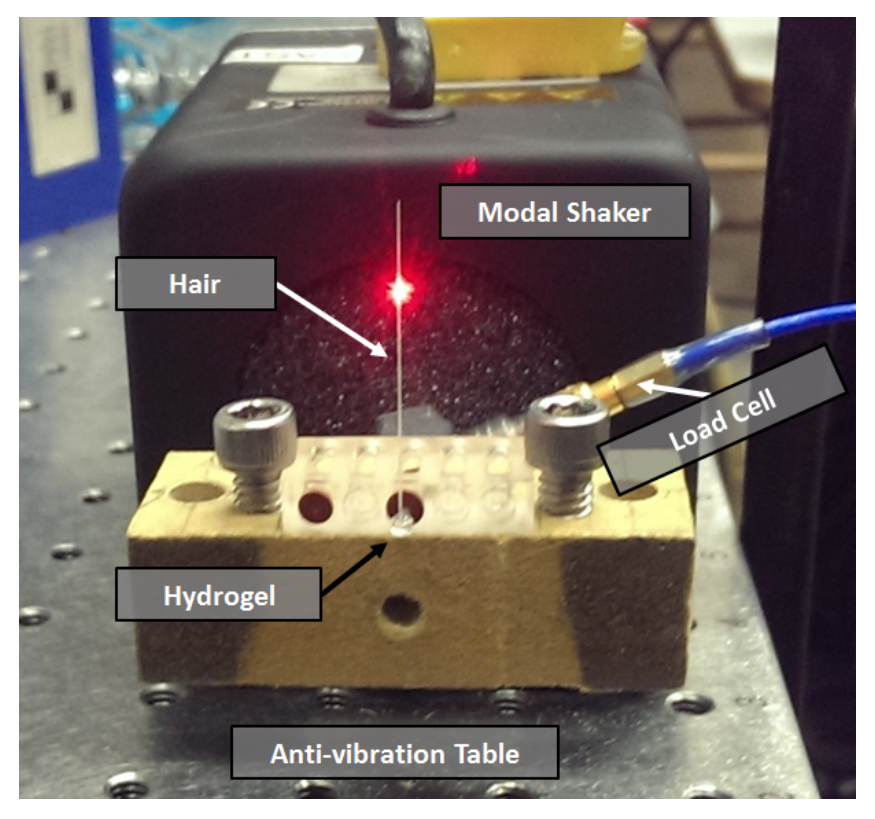

Figure 2.6: A base excitation test was performed on hair-hydrogel assembly suspended in air through the use of a modified substrate. No oil or lipids were used for this experiment. The modified substrate was mounted to a modal shaker which was supported by an anti-vibration table.

\subsubsection{Constant Airflow Excitation}

Constant airflow excitation involved striking a hair with a steady stream of air, inducing a free vibratory response. This form of excitation simulated a fluid flow more representative of a natural environment. A diagram on the setup for this method is shown in Figure 2.8. Pressurized air was fed out of $3.2 \mathrm{~mm}$ diameter plastic tubing to create wide flow profile (no nozzle was used). The air pressure was varied from 0-12 psi to produce flow velocities from 0-72 m/s. A mass flowmeter monitored the steady-state flow rate of the air. The end of the air tube was held by a sliding clamp which could be moved laterally in order to change the excitation's position. In this way, the method could be used to excite both single and multiple AHCs. Constant air flow excitation of single AHCs is used in the sensitivity analysis portion of the results (Ch. 5). "Sweep testing" of AHC array was used for flow localization studies (Ch. 6). The directionality of the AHC sensors was not addressed in this study, so the airflow was always oriented perpendicular to the bilayer, as in the pulse excitation tests. 


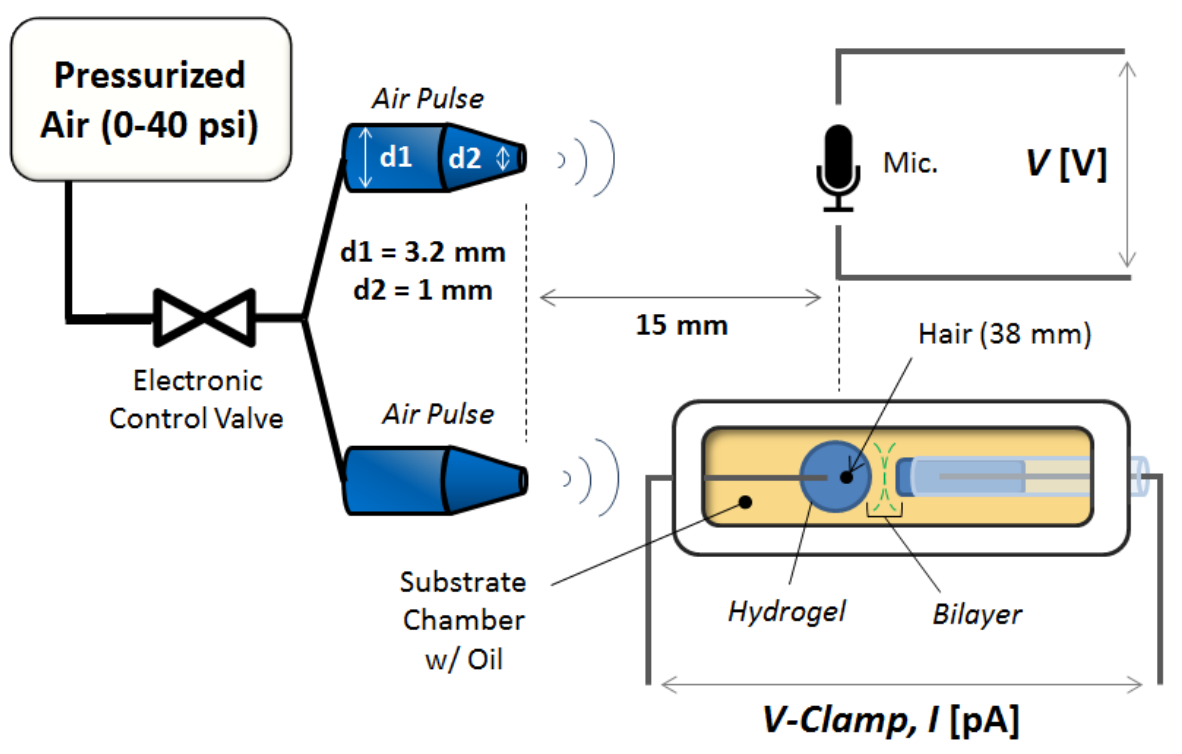

Figure 2.7: A top view of the experimental setup for air pulse excitation shows the pulse's relative orientation to the hair cell unit. The air line was split evenly and placed at a distance of $15 \mathrm{~mm}$ from both hair and microphone. The air pulse was aimed at a height $20 \mathrm{~mm}$ from the base of the hair. To create the pulse, the air was pressurized to 40 psi then released rapidly by opening the valve at $0.5 \mathrm{~ms}$ intervals. The air pressure input to the microphone (measured in volts) was compared to the current output (pA) of the voltage clamped hair cell. 


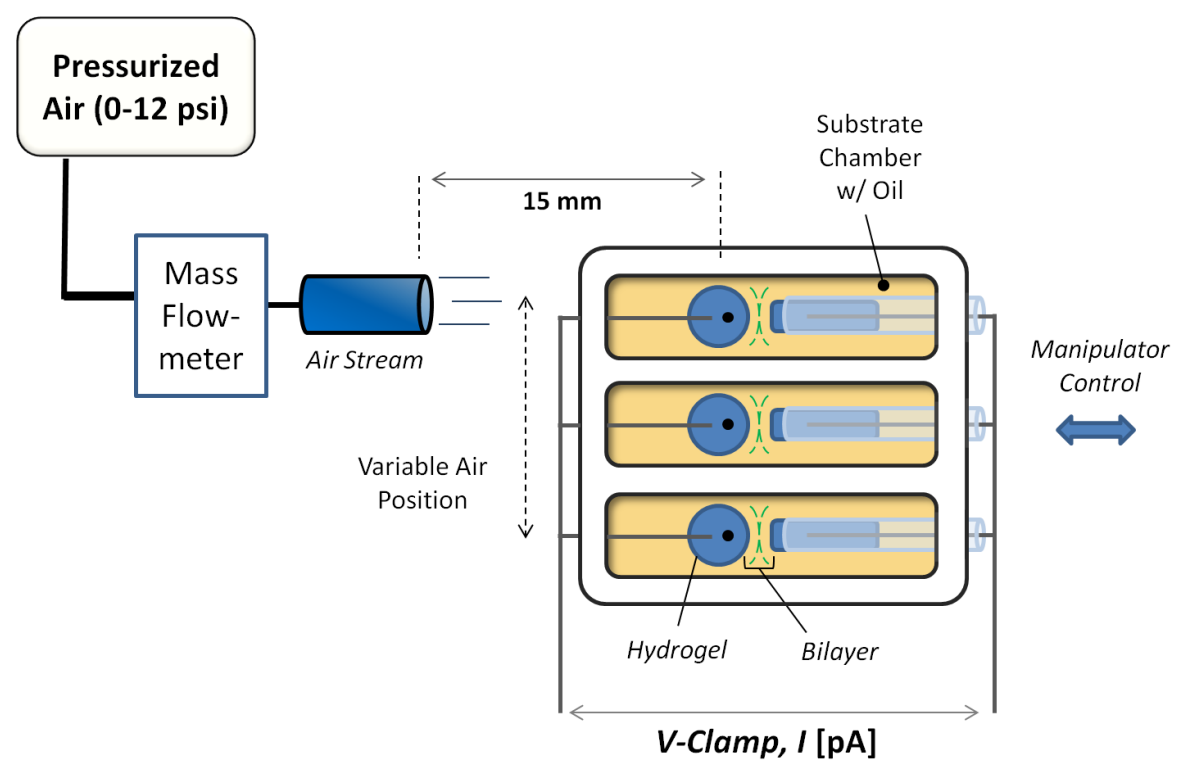

Figure 2.8: A top view of the experimental setup for constant airflow excitation shows the arrangement of the array in relation to the air direction. The air source could be moved laterally to change the excitation position. The air source was aimed at height of $20 \mathrm{~mm}$ from the base of the hair at a distance of $15 \mathrm{~mm}$. A mass flowmeter measured the steady-state flow rate of the air. The output current was the result of the free vibration of the hair(s), measured as described in the 2.2 Experimental Measurements section. 


\section{Chapter 3}

\section{Theory and Modelling}

The sensory mechanics of the AHCs in this study can be divided into two parts: 1) the fluidmechanical interaction between airflow, hair and hydrogel and 2) hydrogel-bilayer transduction. The functional process begins when air flow excites the hair. The hair then vibrates according to the boundary conditions imposed by the hydrogel base. Vibration of the hydrogel itself then excites the bilayer, which produces a current.

For a process such as rapid airflow past a small cylindrical hair, the fluid dynamics can become complex. However, it was observed experimentally that the DC component of constant airflow will deflect the hair while turbulent vortices will excite the hair into vibratory modes around the deflection. Aside from its initial transient, the constant deflection response will have no effect on the response of the bilayer (this is discussed below). Thus, only the vibratory response is significant. Observations of AHC output over a wide range of airflow magnitudes showed remarkable consistency in frequency response, leading to the assumption that the hairs are excited into free vibration, independently of flow velocity.

Another factor to consider is the effect of dynamics of the liquid lipid lenses on bilayer vibration. If sufficiently fluid in the movement, these lenses could add dynamics of their own to alter the frequency response. To circumvent this issue, special care was take experimentally to make the lipid lens coating as thin and close to the hydrogel shape as possible, so that droplet dynamics could be neglected.

In this section we produce theoretical models to better evaluate these interactions. We begin with a mechanical model of the hair-hydrogel system and then transition to an electrical 
description of bilayer transduction.

\subsection{Model of the Hair-Hydrogel System}

The hair is modelled as an elastic beam with boundary conditions imposed by the hydrogel base. THe primary purpose of the model is to predict the natural frequencies of the system and correlate them to the measured frequencies. Each glass hair is assumed to be a tapered beam of uniform density $(\rho)$ and elastic modulus $(E), 2333 \mathrm{~kg} / \mathrm{m}^{3}$ and $71.6 \mathrm{GPa}$ respectively. Assuming a linear taper and circular cross-section, the moment of inertia $I(x)$ can be expressed in terms of its base and tip diameters, $d_{b}$ and $d_{t}$,

$$
I(x)=\frac{\pi}{64}\left(d_{b}-\frac{d_{b}-d_{t}}{L} x\right)^{4} .
$$

The tip is modelled by a free boundary condition while the hydrogel base is modelled by a linear and torsional stiffness constraint as shown in Figure 3.1. The lipid droplet and oil dynamics are ignored, though they may induce damping. Free vibration of the hair is described by the Euler-Bernoulli beam equation for harmonic oscillation, with the appropriate boundary conditions: [92]

$$
\begin{gathered}
\frac{d^{2}}{d x^{2}}\left[E I(x) w^{\prime \prime}(x)\right]=\omega^{2} m(x) w(x) \\
\left.\frac{d}{d x}\left[E I(x) w^{\prime \prime}(x)\right]\right|_{x=0}=-k w(0) \\
\left.E I(x) w^{\prime \prime}(x)\right|_{x=0}=-\tau w^{\prime}(0) \\
\left.\frac{d}{d x}\left[E I(x) w^{\prime \prime}(x)\right]\right|_{x=L}=\left.E I(x) w^{\prime \prime}(x)\right|_{x=L}=0 .
\end{gathered}
$$

Here, $L, w(x)$, and $\omega$ represent the free length, deflection, and natural frequency. The variables $k$ and $\tau$ are the equivalent linear and torsional stiffness of the hydrogel base. Eqn. (3.2) is not solvable analytically due to the varying area moment of inertia $I(x)$. However, the Rayleigh-Ritz approximation method provides an accurate numerical solution [92. First, we multiply both sides of Equation $(3.2)$ by $w(x)$, apply the boundary conditions, and integrate over the length of the hair to yield the Rayleigh quotient $(R)$, which is also equal to the 


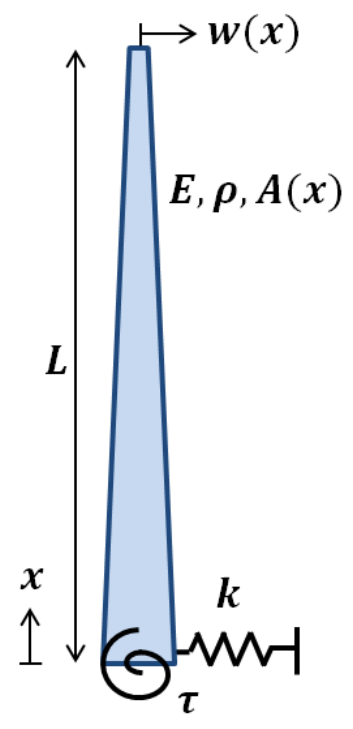

Figure 3.1: Model of the hair-hydrogel system as a uniform, linearly tapered beam with torsional and linear stiffness at the base.

system's natural frequency squared,

$$
R[w(x)]=\omega^{2}=\frac{\int_{0}^{L} E I(x)\left[w^{\prime \prime}(x)\right]^{2} d x+k w(0)^{2}+\tau y^{\prime}(0)^{2}}{\int_{0}^{L} m(x) w(x)^{2} d x} .
$$

The stationary principle states that finding a mode shape $w(x)$ that minimizes the Rayleigh quotient will result in a natural frequency of the system. To do so, we approximate the hair's mode shape to be a linear combination of $n$ admissible functions:

$$
w(x) \approx \mathbf{c}^{T} \phi=\sum_{i=1}^{n} c_{i} \phi_{i} .
$$

Where $\mathbf{c}$ is a vector of scalar weights and $\phi$ is a vector of admissible functions, both of size $n x 1$. Minimizing Eqn. (3.3) with respect to the assumed mode shapes in Eqn. 3.4 yields the approximate eigenvalue problem 


$$
\begin{gathered}
K \mathbf{c}-\omega^{2} M \mathbf{c}=0 \\
K_{i j}=\int_{0}^{L} E I(x) \phi_{i}^{\prime \prime}(x) \phi_{j}^{\prime \prime}(x) d x+k \phi_{i}(0) \phi_{j}(0)+\tau \phi_{i}^{\prime}(0) \phi_{j}^{\prime}(0) \\
M_{i j}=\int_{0}^{L} m(x) \phi_{i}(x) \phi_{j}(x) d x
\end{gathered}
$$

where $K$ and $M$ are $n \times \mathrm{x} n$ generalized stiffness and mass matrices of the approximated dynamic system. For our case, we chose $n=8$ and a set $\phi$ of sine and cosine functions that met the problem's geometric constraints. The stiffness values $k$ and $\tau$ are fitted experimentally to match the first two natural frequencies of a given system. The model accuracy is then evaluated by how well it approximates additional frequencies. It was found that imposing simply a 1 degree of freedom boundary condition (i.e. just a linear or torsional stiffness constraint) was not enough to properly approximate the hair's natural frequencies.

\subsection{Bilayer Mechanoelectrical Transduction}

If we assume negligible droplet dynamics, then bilayer vibration will occur at the natural frequencies of the hair, with modal magnitudes similar to those near the beam's base. Since bilayer deflection changes its electrical properties, we begin with an electrical model of the bilayer.

\subsubsection{Circuit Representation}

The electrical circuit representation of a droplet interface bilayer (DIB) is shown in figure 3.2. Two volumes of electrolyte solution are immersed in a non-conductive oil phase. The electrolyte solution encompasses both the aqueous lipid solution and the solid hydrogel scaffolding in our study; they are represented as single volumes in the figure for clarity. The electrolyte contains dissociated ions of potassium chloride $(\mathrm{KCl})$, which allow it to conduct charge. Each volume is pierced by a measurement electrode. The bilayer, which occurs at the interface of the electrolyte volumes, is the only conductive path for the ions to travel. An ideal bilayer is impermeable to ionic flow, but it is thin enough to allow charges to accumulate on either side, just like a capacitor. In reality, however, bilayers have a finite, albeit 


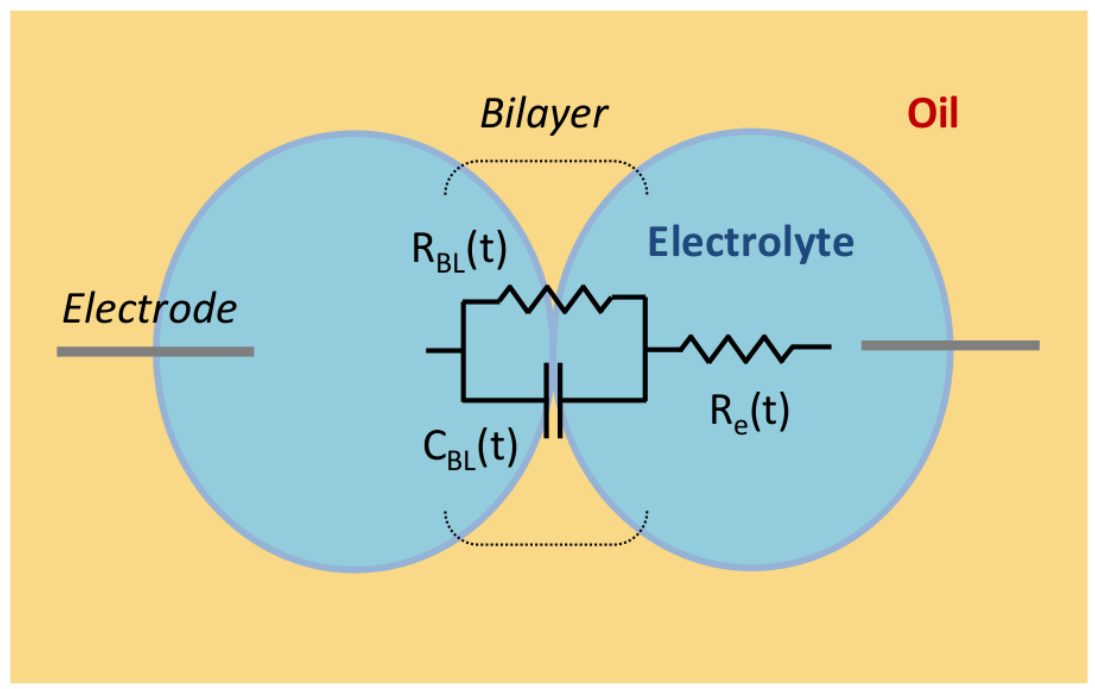

Figure 3.2: Electrical circuit representation of a droplet interface bilayer.

high resistance. Well-formed bilayers typically have resistance values in the gigaohm range [93]. Thus, the bilayer can be modelled as a resistor $R_{B L}$ and capacitor $C_{B L}$ in parallel, as in figure 3.2. The electrodes and electrolyte on either side of the bilayer also contribute a small resistance and this is represented as a single series resitor $R_{e}$. Charge may build up at the electro-electrolyte interface to create stray capacitance, however the values for reversible $\mathrm{Ag} / \mathrm{AgCl}$ electrodes are negligible.

\subsubsection{Current Generation Through Vibration}

Current is induced in the circuit by hair vibrations, which are transferred to the membrane and cause it to bend. Bending causes both curvature and tension in the membrane. In Chapter 1, we established three forms of current generation due to these effectst? Mechanoconductance, flexoelectricity, and mechanocapacitance. Mechanoconductance is only possible with the presence of mechanosensitive proteins which are not used this study. Flexoelectricity is possible in pure lipid bilayers, however, it is a significant effect only for charged lipids and so can be safely ignored. This narrows our scope to the effects of mechanocapacitance.

Our circuit model suggest that the total current $(i)$ across the bilayer has two components:

\footnotetext{
${ }^{1}$ Note that electrowetting, although grouped under mechanoelectrical transduction, is not strictly a form of current generation.
} 

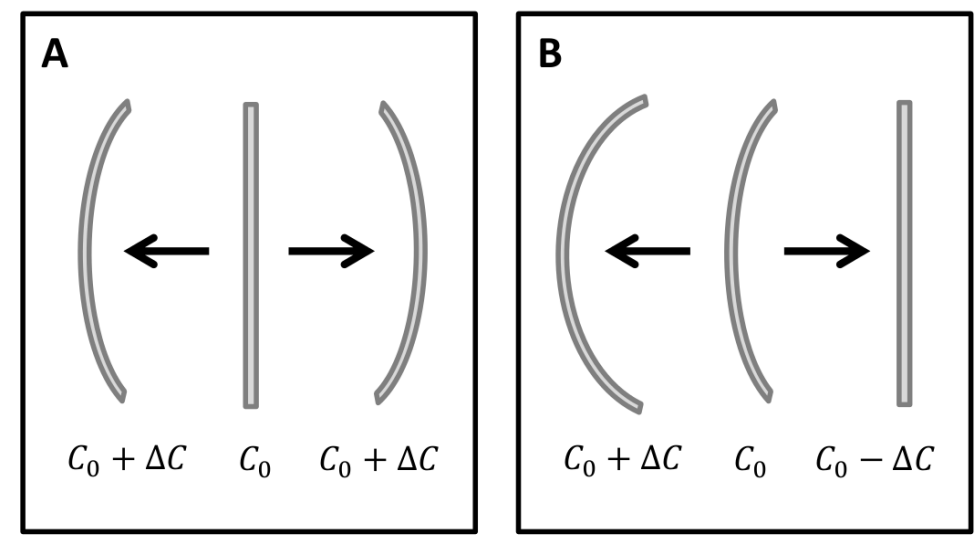

Figure 3.3: Two variations of mechanocapacitance. A) Capacitance change due to membrane bending around a planar configuration. Capacitance increases in both directions. B) Capacitance change due to membrane bending around a pre-bent configuration. Capacitance increases with increased curvature and decreases as membrane becomes planar.

a resitive current $\left(i_{R}\right)$ and a capacitive current $\left(i_{C}\right)$. They are described as follows

$$
\begin{array}{r}
i_{R}=V / R_{B L} \\
i_{C}=\frac{d}{d t}\left(V C_{B L}\right) \\
i=i_{R}+i_{C}
\end{array}
$$

where $V, R_{B L}$ and $C_{B L}$ represent voltage, bilayer membrane resistance and capacitance, respectively. Since membrane oscillations change the surface area of the membrane, both resistance and capacitance are expected to change. However, large bilayer resistance $(>10$ $\mathrm{M} \Omega$ ) results in small values of $i_{R}$ such that even relatively slow changes in capacitance will begin to overshadow it. In fact, similar membranes to the ones in this study demonstrate purely capacitive behavior between excitation frequencies of 1-10,000 Hz. The hair vibrational frequencies studied here fall well inside this range, thus we will consider $i \approx i_{C}$. For a constant voltage applied to the bilayer, equation 3.6 simplifies to:

$$
i=V \frac{d}{d t}\left(C_{B L}\right)
$$

The principle of mechanocapacitance states that capacitance will increase with membrane curvature, due to the increase in surface area. The effect is insensitive to the direction of 
curvature. Two variations on this phenomenon exist, as shown by Figure 3.3. In the first case (Figure 3.3A), the membrane oscillates about a planar configuration, reaching a maximum capacitance twice per oscillation period. The time-dependence of the membrane capacitance can be expressed as a sinusoidal change at twice the stimulus frequency,

$$
C_{B L}(t)=C_{m} \sin (2 \omega t)+C_{p i}
$$

where $C_{m}$ is the maximum change in membrane capacitance and $C_{p i}$ is the initial, undisturbed capacitance of the planar membrane. In the second case (Figure 3.3 B), the membrane oscillates about a bent configuration, alternating between one minimum and maximum capacitance. The, the time-dependence of the capacitance matches the stimulus frequency,

$$
C(t)=C_{m} \sin (\omega t)+C_{b i}
$$

where $C_{b i}$ is the undisturbed capacitance of the bent membrane, which is larger than $C_{p i}$. Considering the second case and inserting equation 3.9 into equation 3.7, we get the current dependence on an oscillating capacitance:

$$
i=V \omega C_{m} \cos (\omega t)
$$

From this equation we note four things about the bilayer output current: 1) it is proportional to voltage, 2) it is proportional to stimulus frequency, 3) it is proportional to the amplitude change capacitance and 4) it is offset by 90 degrees from the membrane deflection [36].

\subsubsection{Electrowetting Effect}

In using equation 3.10, one must take into account that capacitance is also a function of the applied voltage due to the electrowetting effect. This effect, explained in Chapter 1, states that an applied electric field will reduce the contact angle between two aqueous volumes to produce a larger contact area [56]. Due to this increase in area, it has been established experimentally that bilayer capacitance $C$ will vary with the square of the applied voltage $V$ as in Eqn. (3.11) [94, 95]. 


$$
C(V, t)=C_{0}(t)\left(1+\alpha V^{2}\right)
$$

Here, $C_{0}$ is the capacitance at zero volts and $\alpha$ is the voltage dependence, which is sensitive to both lipid type and bilayer geometry. Replacing $C_{0}$ with equation 3.9 and substituting equation 3.11 into equation 3.7, we get a more complete expression for the total membrane current:

$$
i(V, t)=\omega C_{m 0} \cos (\omega t)\left[V+\alpha V^{3}\right]
$$

where $C_{m 0}$ is membrane maximum capacitance change at zero volts. From this relation we notice that electrowetting gives the bilayer membrane a cubic voltage sensitivity. The voltage sensitivity, however, is only an amplification factor for the membrane output at zero volts and should not, in theory, change its frequency output characteristics. 


\section{Chapter 4}

\section{Characterization of AHC Sensor's Dynamic Response}

The membrane based AHC sensor is a complex system both electrically and mechanically. Electrically, it is susceptible to many different sources of noise, making it critical to separate these sources from the current contributions of the bilayer membrane. Because the membranes are delicate biological structures, their electrical properties may vary more than traditional components. Such variability must also be taken into account. Mechanically, the sensor is a dynamic system composed of a beam (the hair) with a complex base condition (the hydrogel) coupled to a fluid membrane with dynamics of its own. Membrane dynamics could be affected by lipid lens sizes, contact angle and orientation, as well as adhesion to the hydrogel support. In the context of sensing flow characteristics, an ideal lipid membrane should be very responsive to the hair's vibrations without contributing major dynamics of its own. The purpose of this section is to begin by analyzing the dynamic behavior of the hair and hydrogel alone and then examine how well this behavior is reflected in the membrane current output. The former is achieved through a base excitation method and the latter through air impulses. The results show that the hairs vibrate at various bending modes and that low noise design of the AHC allows for great coherence when characterizing the input-output relationship from hair vibration to membrane current. 


\subsection{Control Testing}

\subsubsection{Sources of noise}

Experimental tests on AHCs with no bilayers have shown two principal sources of undesired spectral noise in the output signal. These non-bilayer signals can contaminate the frequency output of the bilayer itself and cause errors in response interpretation. The first noise source was low-level power-line interference from instrumentation. The second type of noise, electrode noise, arose primarily from mechanical vibrations of the measurement electrode connected to the positive terminal of the Axopatch headstage. In order to understand these noise sources better and learn how to mitigate them, we subjected AHCs assembled with no bilayers (open circuit, plain hydrogels $\sim 2 \mathrm{~mm}$ apart) to both airflow and voltage variation. Figures 4.1 and 4.2 illustrate the results.

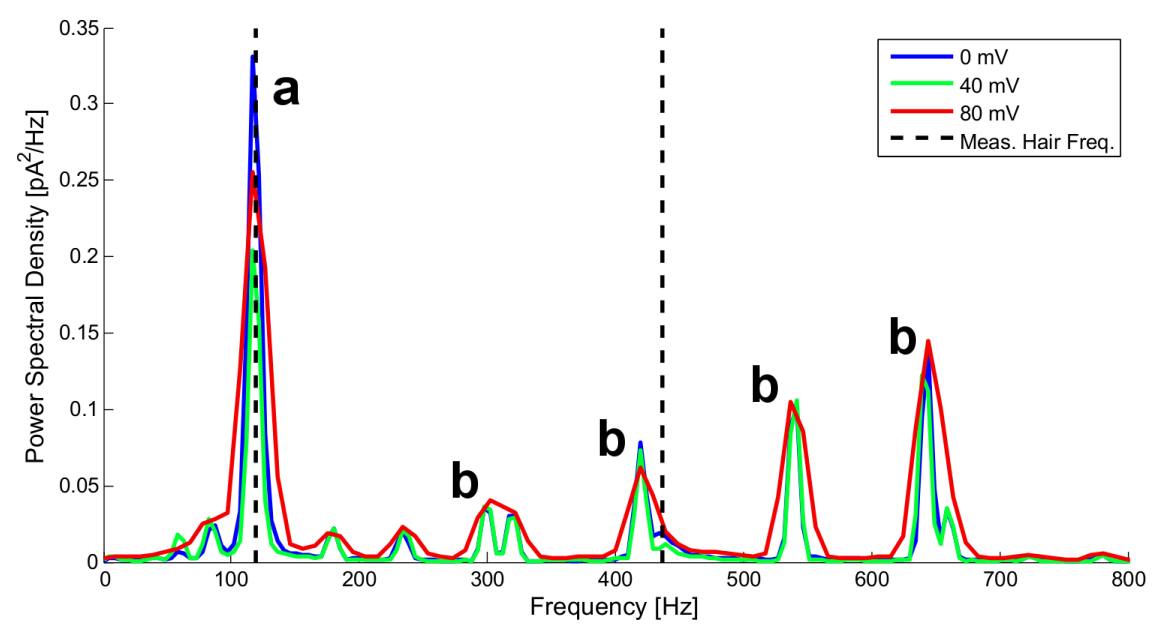

Figure 4.1: Voltage dependence of both types of electrical noise: electrode noise (a) and powerline noise (b). Data is a result of measuring airflow current response (at a constant $34 \mathrm{~m} / \mathrm{s}$ ) of an $\mathrm{AHC}$ with no bilayer. Colored lines denote the AHC applied voltage. Dotted lines indicate the experimentally measured natural frequencies of the hair.

Power line noise identified spectrally by sharp peaks at scalar multiples of $60 \mathrm{~Hz}$ (see either Figure 4.1 or 4.2. The highly frequency-correlated nature of this noise source made is troublesome to interpret the power spectral density content of low amplitude signals. It can be reduced significantly by shielding the AHCs within a Faraday cage and grounding all conductive components inside that may act like antennas. When properly shielded, the noise 
was relatively constant through all voltages (Figure 4.1) and airflows (Figure 4.2). Additional tests (not shown) confirmed that this holds even in the presence of a bilayer. Based on its overall behavior, electrical interference is consider a property of the instrumentation and how well it is shielded. Its spectral contribution varied between $<0.2 \mathrm{pA}^{2} / \mathrm{Hz}$ for well-shielded cases and up to $2 \mathrm{pA}^{2} / \mathrm{Hz}$ for high-interference cases.

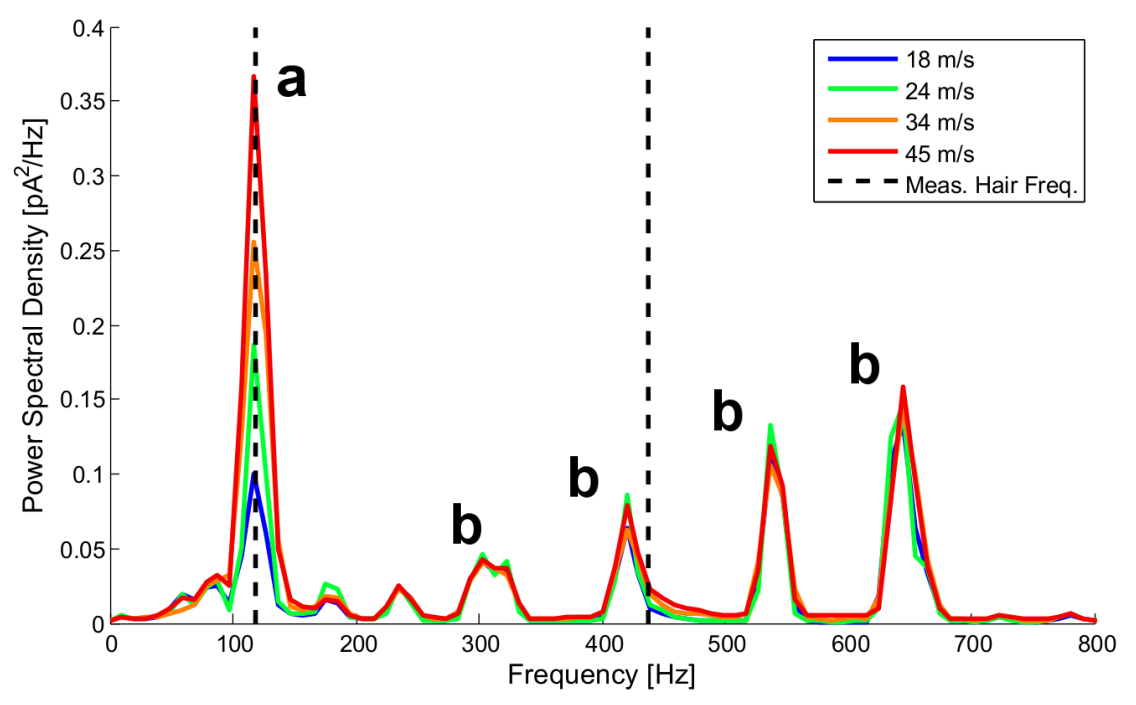

Figure 4.2: Air velocity dependence of both types of electrical noise: electrode noise (a) and power-line noise (b). Data is a result of measuring airflow current response of an AHC with no bilayer at $80 \mathrm{mV}$ as the air velocity is increased incrementally from 18 to $45 \mathrm{~m} / \mathrm{s}$ (colored lines). Dotted lines indicate the experimentally measured natural frequencies of the hair.

Electrode noise is a result of the extreme current sensitivity of the measurement instrumentation, and thus could be introduced by movements of the hair itself on the electrodes. This meant that hair vibration could cause sensory output, at its characteristic frequencies no less, even in the absence of a bilayer. The hairs' vibrational contribution to electrode noise tended to vary significantly from experiment to experiment in the $1-10 \mathrm{pA}^{2} / \mathrm{Hz}$ range. Although the exact mechanism for current production is not yet understood, it could be partially mitigated by insulating electronic components well, minimizing unnecessary mechanical vibrations and connecting the positive electrode to the secondary hydrogel, rather than to the one which held the hair. For an open AHC with no bilayer, it was observed that electrode noise varied little with applied voltage (Figure 4.1) but increased with airflow velocity (Figure 4.2), just as the mechanical vibration of the hair. Notice how in the figures, despite measure natural frequencies measured at 120 and $437 \mathrm{~Hz}$ (dotted lines), the electrode noise is limited to the 
lower frequency. This result was typical; electrode noise only seemed to occur below $200 \mathrm{~Hz}$.

\subsubsection{Bilayer versus no bilayer}

To determine if the noise mitigation measures in this work were adequate to study bilayer sensory output, we tested several AHCs before and after bilayer formation. We will refer to these cases as no bilayer and bilayer, respectively. Both configurations were tested at various voltages and airflow velocities, shown in Figure 4.3. The root mean square (RMS) amplitude of the current output signal was used a comparison of the overall magnitude of the signal. The figure shows that the response magnitude for the bilayer cases (diamonds) noticeably increased with both voltage and air velocity. The no bilayer case, on the other hand, showed only a very slight increase with air velocity (due to small amounts of electrode noise) but none with voltage. In the illustrated case, even at $0 \mathrm{mV}$ and low airflow, the bilayer output showed nearly $400 \%$ increase over the noise floor. In general, the results suggest that we can greatly improve AHC signal to noise ratio by amplifying the signal (and not the noise) through an applied potential. 


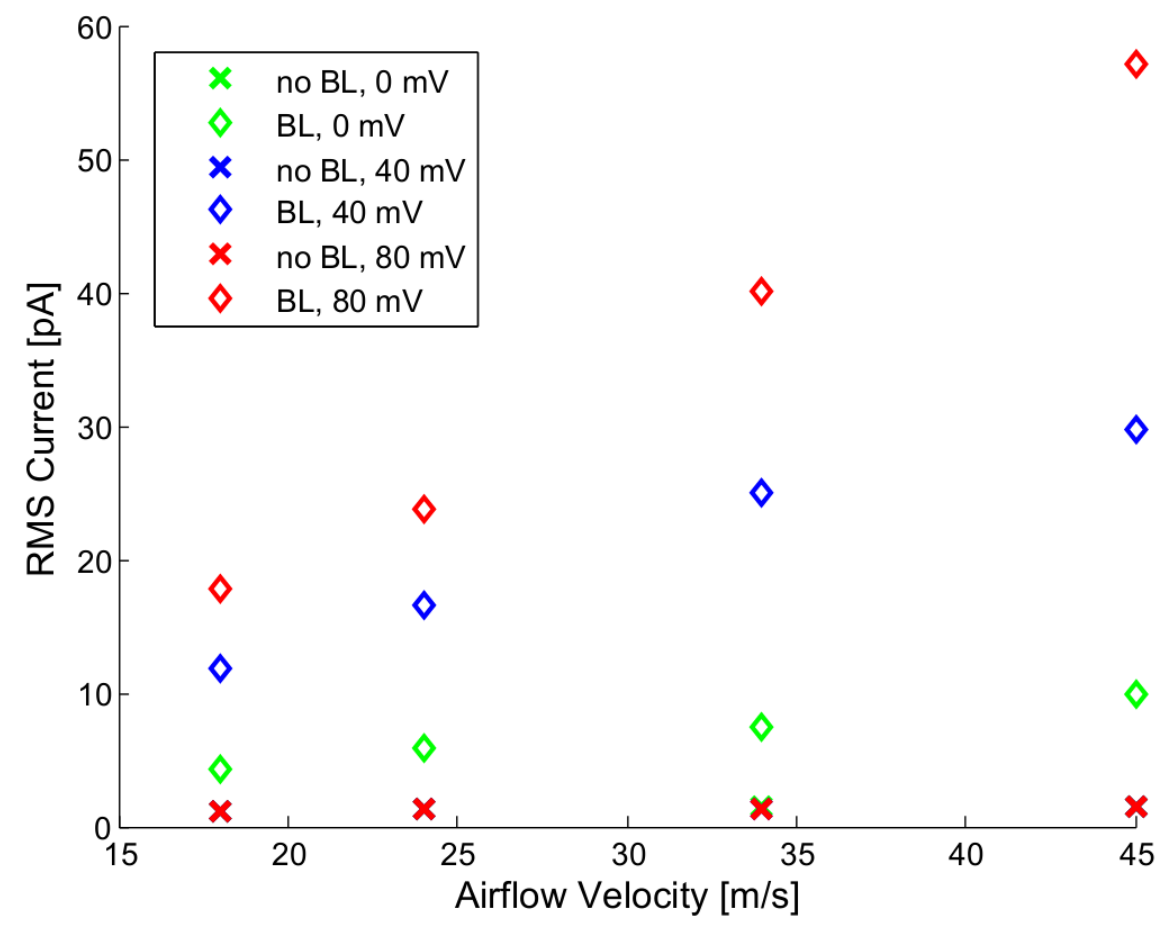

Figure 4.3: Control analysis of the AHC output for bilayer and no bilayer cases as both voltage and air velocity are increased. The root mean square (RMS) amplitude of the current output signal was used a comparison of the overall magnitude of the signal.

\subsubsection{Vibration isolation between AHC units}

The final control parameter to consider was the vibrational interplay between AHCs in arrays. In other words, how sensitive are bilayers to the mechanical vibrations not from their corresponding hair? To study this, tests were conducted for two adjacent AHCs, one with a bilayer and one without, hair 1 and hair 2 respectively in Figure 4.4. Three flow configurations were tested: 1) airflow directly at hair 1,2) airflow directly at hair 2 and 3) airflow at both hairs.

After various tests we observed that flow on hair 1 (with bilayer) yielded high, well-defined spectral output corresponding to hair vibrations, as was expected. Flow over only hair 2 (adjacent hair) manifested as a low current contributions the output of AHC 1, slightly above the noise floor (see Figure 4.5). The leakage was confirmed to occur at one of the natural frequencies of hair 2 (dotted lines). The magnitude of the contribution was dependent on both voltage and air velocity, but only noticeable for large voltages $(>60 \mathrm{mV})$ and airflows 


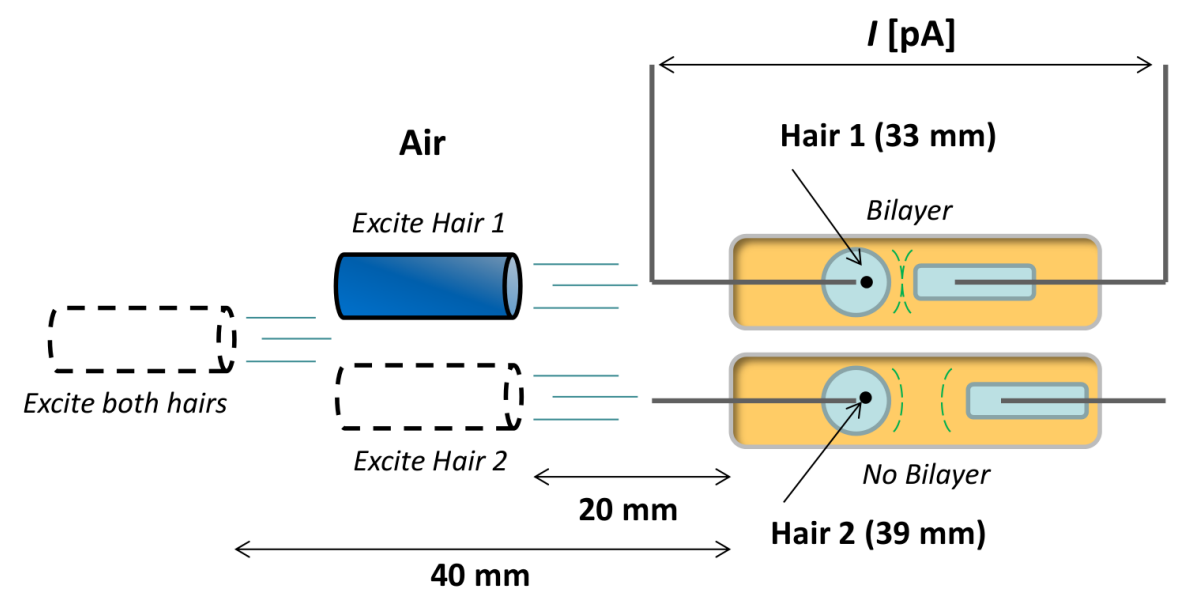

Figure 4.4: Experimental setup for array isolation control experiments. The output of the top AHC was monitored to observe any vibration leakage from the bottom AHC, which was not connect. Three different airflow positions were used: 1) airflow directly at hair 1,2) airflow directly at hair 2 and 3) airflow at both hairs.

$(>30 \mathrm{~m} / \mathrm{s})$. Even so, it typically did not exceed $10 \mathrm{pA}^{2} / \mathrm{Hz}$. The voltage and airflow dependence of the signal supports the theory that stray hair 2 vibrations are picked up by the bilayer in AHC 1. Another fact that supports this theory is that only higher modes are picked up; recall from equation 3.12 that bilayer output scales with frequency.

Finally, we observed that the AHC 1 output dominates when both hairs are excited as seen in Figure 4.6. Notice how no contributions are seen at the hair 2 natural frequencies (dotted lines). We conclude overall that these results demonstrate good vibration isolation between adjacent AHCs and that stray vibrations may be neglected. 


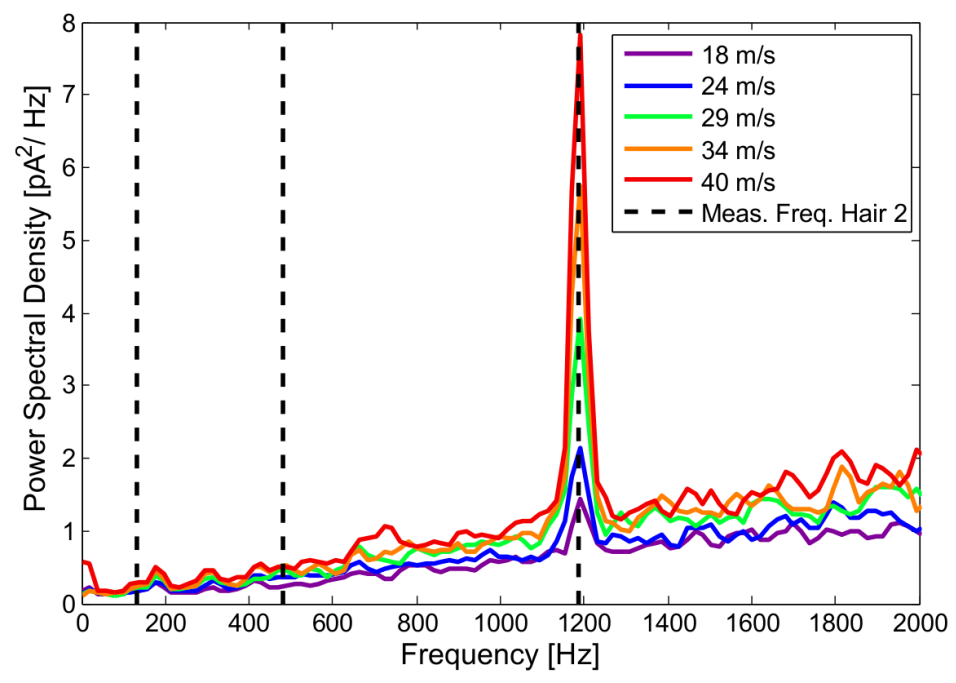

Figure 4.5: Vibration isolations test where an AHC output is measured while an adjacent AHC is excited. In this case, the measured AHC was held at $80 \mathrm{mV}$ while the adjacent hair was excited incrementally from 18 to $40 \mathrm{~m} / \mathrm{s}$ airflow. Output shows that small vibrations are picked up only at the third of the adjacent hair's natural frequencies (dotted lines). Refer to Figure 4.4 for more information.

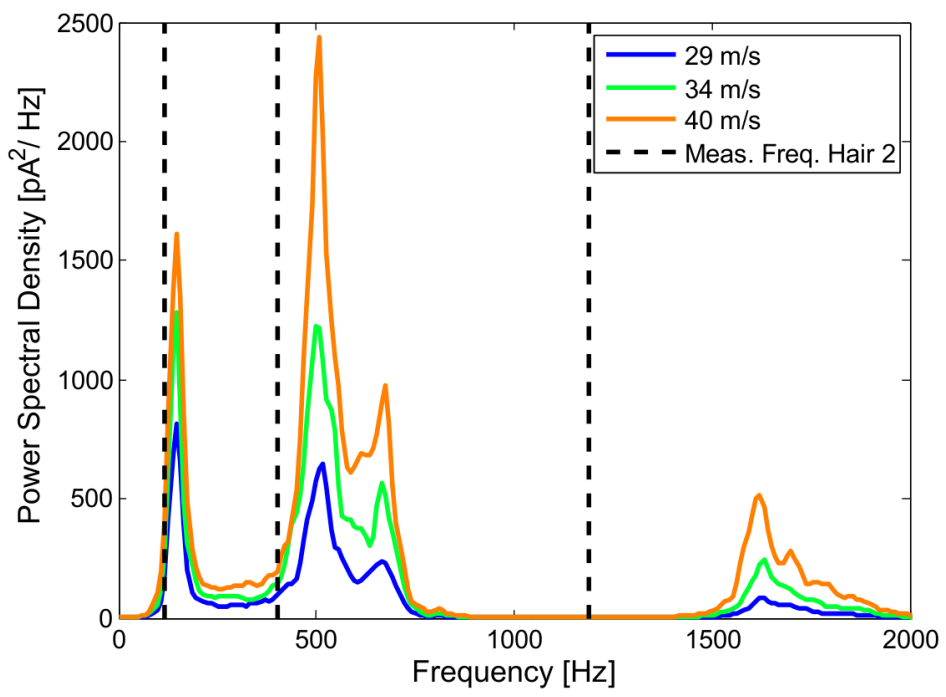

Figure 4.6: Vibration isolations test where both a connected and unconnected AHC where excited. In this case, the measured AHC was held at $80 \mathrm{mV}$ while the adjacent hair was excited incrementally from 29 to $40 \mathrm{~m} / \mathrm{s}$ airflow. Output shows that the natural frequencies of the unconnected hair (dotted lines) are overshadowed by the output of the connected AHC. Refer to Figure 4.4 for more information. 


\subsection{Base Excitation Results}

In order to better understand the dynamics of the hair alone, an experimental hair-hydrogel assembly with no membrane was scanned with a laser vibrometer to determine its frequency response and mode shapes. A 5-2,000 Hz sweep base excitation was applied. The resulting hair velocity/load transfer function estimate, seen in Figure 4.7, reveals five resonant frequencies $(31,170,661,804$, and $1692 \mathrm{~Hz})$ within the $2 \mathrm{kHz}$ spectrum. Figure 4.8 shows the mode shapes corresponding to each frequency. The rigid body mode at $31 \mathrm{~Hz}$ is a result of the induced base motion due to the excitation technique. The rest are pure bending modes of the structure. Two distinct second bending modes are observed (661 and $804 \mathrm{~Hz}$ ), similar to what Jampole et al. noticed for the first bending mode in fiber hairs [96]. Since the vibrometer measures only one axis of vibration, this could be a result of two normal modes vibrating at an angle to this axis. Alternatively, a coupling of two nearby modes may be occurring. Either of these phenomena are likely due to non-symmetry, both in the position of the hair on the hydrogel as well variability in the hydrogel mechanical properties. The net effect is stiffness that varies with angular position, which could easily cause a divergent mode as seen here. The split mode has only been observed in the second mode and is quite common, but occurs at various degrees of magnitude.
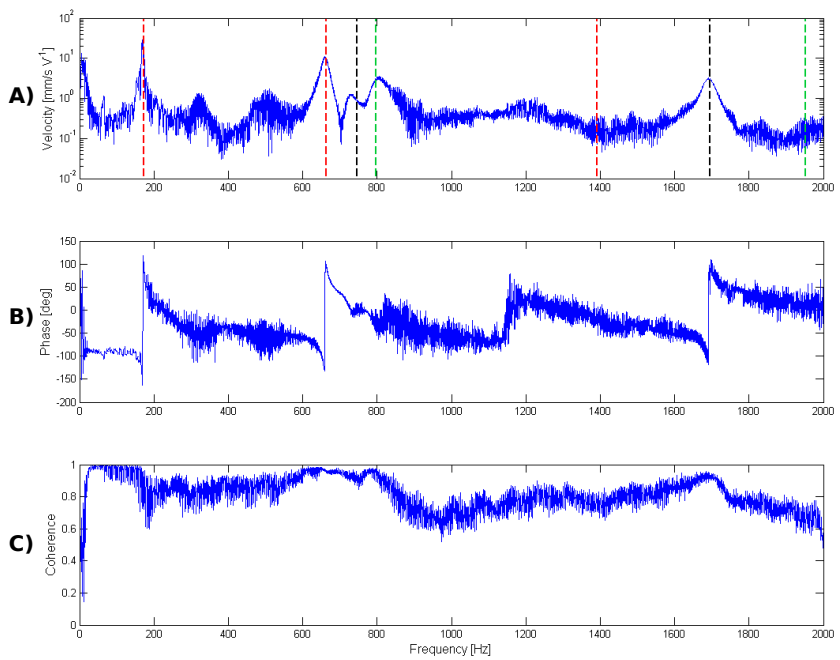

Figure 4.7: A) Average spectrum transfer function magnitude (hair velocity $(\mathrm{mm} / \mathrm{s}) /$ shaker load $(\mathrm{V})$ ). Dotted lines show model frequency approximations for $t=0.0018 \mathrm{Nm} / \mathrm{rad}$ and $k=39 \mathrm{~N} / \mathrm{m}$ (red), $k=98 \mathrm{~N} / \mathrm{m}$ (black), and $k \approx 200 \mathrm{~N} / \mathrm{m}$ (green). B) Average spectrum phase. C) Coherence plot shows a high signal to noise ratio throughout. 


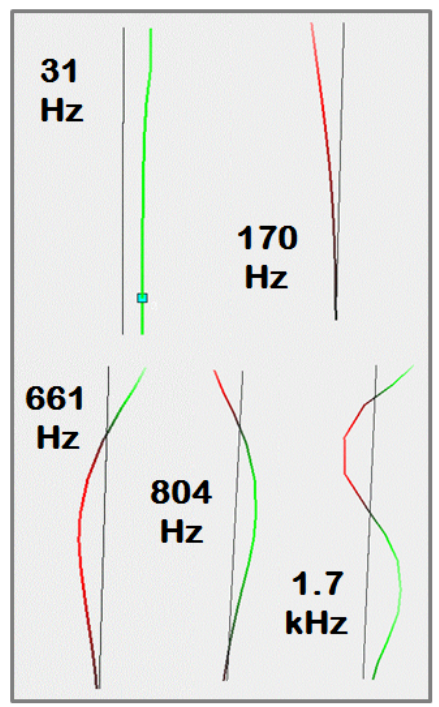

Figure 4.8: Experimentally determined mode shapes of the hair-hydrogel assembly.

Note that the spring boundary conditions in Eqn. (3.2) are decoupled by definition; this results in a decoupling of the first and second modes so that they are not heavily influenced by either the torsional or linear stiffness, respectively. The double second bending mode suggests that equivalent linear stiffness of the hydrogel may be non-uniform around the hair. This non-ideal case cannot be predicted by our 2 DOF stiffness model, but may be explored by testing various values of linear stiffness. Choosing gel stiffness values $(k=39$ $\mathrm{N} / \mathrm{m}$ and $\tau=0.0019 \mathrm{Nm} / \mathrm{rad}$ ) so that Eqn. (3.5) matches the first two bending frequencies results in a third mode prediction that is about $300 \mathrm{~Hz}$ lower than the measured mode (red lines in Fig. 4.7A). This is not an accurate prediction, even in consideration of experimental measurement error of hair dimensions. On the other hand, adjusting the linear stiffness $(k=200 \mathrm{~N} / \mathrm{m})$ to match the double mode at $804 \mathrm{~Hz}$ yields a third mode that is about 300 $\mathrm{Hz}$ too high. However, a middle choice of linear stiffness ( $k=98 \mathrm{~N} / \mathrm{m}$, black lines), results in much better agreement, with a second mode that lands between the split experimental modes. Although the precise dynamics are still uncertain, this result suggests that the third mode could depend on coupled effects between the two linear stiffnesses. It is also possible that a double third bending mode exists outside of the measured frequency range.

We also chose to measure hair velocity magnitude, rather than displacement, because of its relation to membrane current output. Membrane current $i_{m}$ is related to the rate of capacitance change. Thus, for an oscillating capacitance $C(t)=C_{0}+C_{1} \sin (\omega t)$, it is scaled 
by the frequency of oscillation $\omega[55]$.

$$
i_{m}(t)=V C_{1} \omega \cos (\omega t)
$$

Since we expect hair and membrane displacement to be mechanically linked, it is more informative to track the hair velocity for comparison with membrane current. Fig. 4.7A shows how the average spectrum hair velocity is highest at the first mode and lowest at the third.

\subsection{Air Pulse Excitation Results}

Sharp air pulses, approximating an impulse excitation, were used to characterize the fully assembled AHC behavior. Figure 4.9 demonstrates typical time domain signals for both membrane current and air pulse. Note the impulse-like behavior of the input pulse (Fig. 4.9B) and the oscillatory ring-down of the AHC's output response (Fig. 4.9 A). Peak currents regularly registered in the nanoamp range as seen here. To the authors' knowledge, previous membrane based AHCs have shown output currents of only a few hundred picoamps. Such excellent signal strength, given that electrical noise accounts for less than $1 \mathrm{pA}$ of the current output, provides great clarity in our characterization results. Figure $4.10 \mathrm{~A}$ shows a transfer function estimate for a pulsed hair of similar dimensions as the base excitation experiment. Despite the different excitation method, results show the three bending modes with excellent coherence (Fig. 4.10B). A rigid mode is not observed due to the nature of the excitation. The roundedness of the peaks suggests heavy damping, likely due to the presence of oil. Divergence is observed once again at the second mode, though not as distinct as in the base excitation case.

Fitting the Eqn. 3.5 model to the first two modes does an excellent job of predicting the third, as demonstrated by the dotted red lines in Fig. 4.10A. The fit's estimated stiffness values ( $k=19 \mathrm{~N} / \mathrm{m}$ and $\tau=0.0011 \mathrm{Nm} / \mathrm{rad}$ ) show a $42 \%$ and $51 \%$ decrease from those calculated in the base excitation experiment. As the oil's damping effect would tend to decrease the hair's natural frequencies, this decrease could be the model's form of compensation.

Note the magnitude dominance of modes 2 and 3 in Figure 4.10, contrasting with the dominance of mode 1 for the base excitation average spectrum in Fig. 4.7. The difference in 

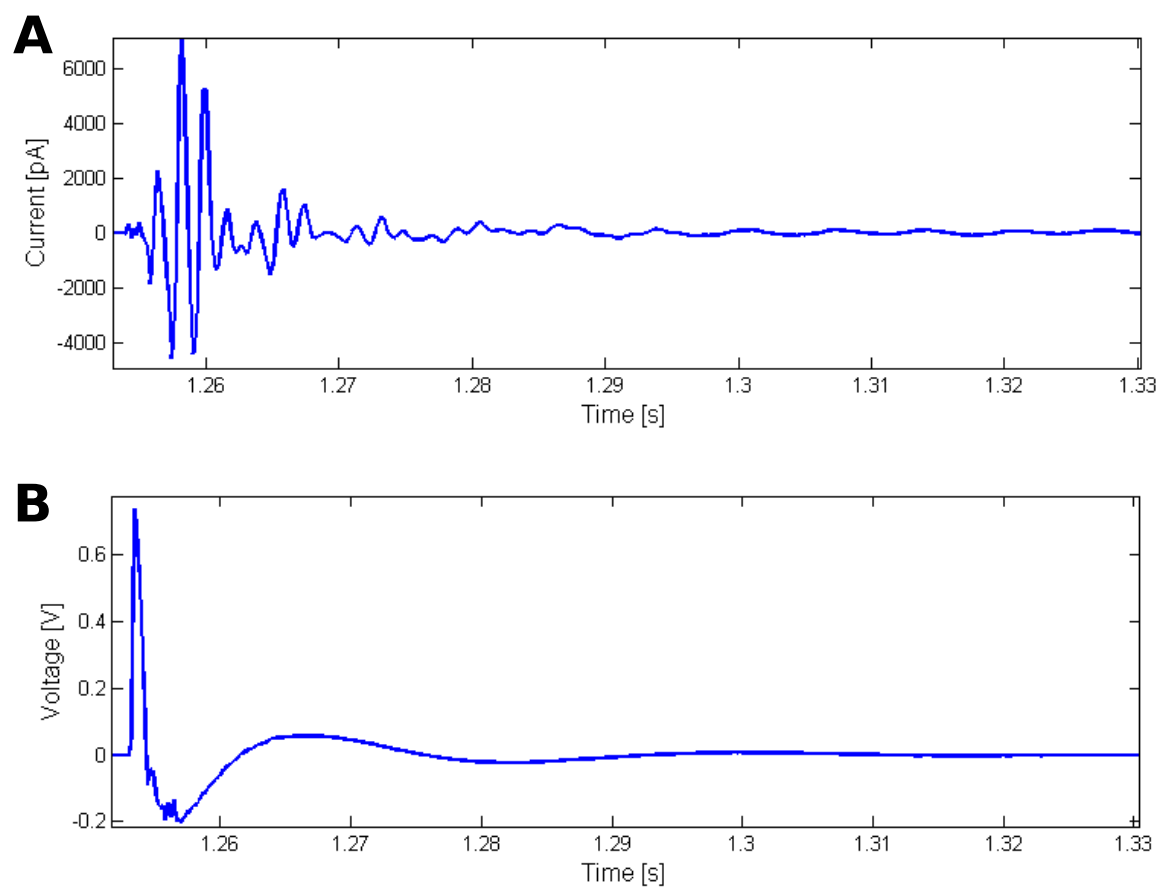

Figure 4.9: A) Membrane current output versus time after hair pulse excitation (AHC system output). B) Pressure microphone voltage output versus time (AHC system input).

ratios among mode magnitudes suggests that the membrane current is not reflective of the vibrations across the entire hair. A qualitative look at modes shapes (Fig. 4.8) show that hair velocity (i.e. distance from the neutral axis) is greatest for mode $2(661 \mathrm{~Hz})$ and mode 3 $(1.7 \mathrm{kHz})$ towards the base of the hair. This implies that the membrane capacitance change depends primarily on vibrations closest to the hydrogel, as one would expect. 

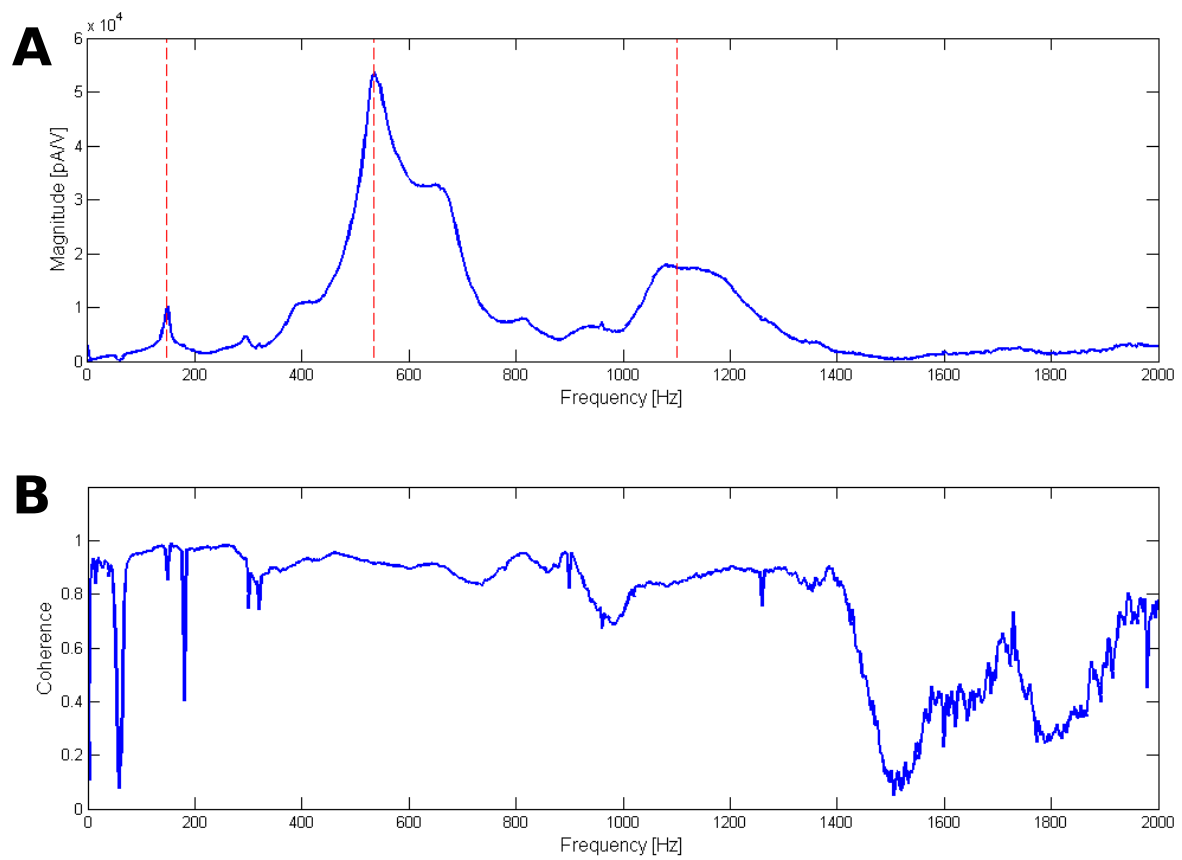

Figure 4.10: A) Transfer function estimate (output current (pA)/pulse pressure (V)) for a pulse excitation. Dotted red lines indicate model frequency approximation for $k=19 \mathrm{~N} / \mathrm{m}$ and $\tau=0.0011$ $\mathrm{Nm} / \mathrm{rad}$. B) Signal coherence. 


\section{Chapter 5}

\section{AHC Sensitivity Properties}

While the previous chapter studied how the hair's dynamics were reflected the membrane's output, this chapter explores the overall sensor's sensitivity to changes in the input. The three available inputs to AHC sensor were the clamping voltage, the magnitude and the position of the airflow. Understanding the sensor's response to variations in these inputs helps to interpret information about the external environment. From this perspective, it is important to consider not only how the magnitude of the output is affected, but also the frequency response. The author first explores how special properties of lipid bilayer membranes combine with the applied voltage to create an input amplification effect. Secondly, he analyzes how the sensor's output scales with variations in the magnitude and location in the airflow.

\subsection{Sensitivity to Applied Voltage}

\subsubsection{Electrowetting Effect and Voltage Capacitance Relationship}

An applied electric field will reduce the contact angle between two aqueous volumes to produce a larger contact area, a behavior called electrowetting [56]. Due to this increase in area, it has been established that bilayer capacitance $C$ will increase linearly with the square of the applied voltage $V$ as in Eqn. (5.1) [94, 95. 


$$
C(V, t)=C_{0}(t)\left(1+\alpha V^{2}\right)
$$

Here, $C_{0}$ is the capacitance at zero volts and $\alpha$ is the voltage dependence. To test the effects of electrowetting on the AHC sensor sensitivity, we measured the membrane capacitance at various DC voltage offsets as shown in Figure 5.1. The results show a region of linear increase at low voltages followed by a plateau. Fitting Eqn. 5.1 to the low-voltage region yields $\alpha=21.5 \mathrm{~V}^{-2}$. This estimate matches very closely to experimental values of $22 \mathrm{~V}^{-2}$ and $\mathrm{V}^{-2}$ measured by White and Chang [97] and Sarles and Leo [61], respectively.

The plateau region, on the other hand, shows little voltage dependent capacitance ( $\alpha=$ $3.9 \mathrm{~V}^{-2}$ ). This saturation phenomenon has been shown to occur when the geometry and mobility of the aqueous lipid solution is constrained by supporting the lipid droplets such that their natural shape restricted, large scaffolds that are of comparable size to the droplet, and a large DIB diameter relative to the droplet diameter [56]. Such conditions are quite relevant to our case and help to explain why the effects of electrowetting on bilayer dimensions may be limited in our study. Since droplet size and orientation change significantly from test to test, the saturation region may occur at different voltages.

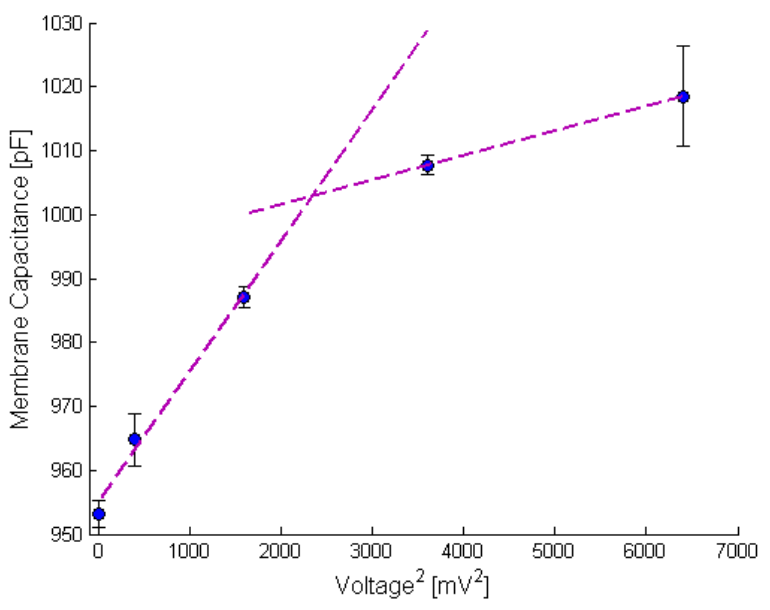

Figure 5.1: Measured membrane capacitance versus the applied voltage squared. Error bars denote \pm 1 standard deviation. Capacitance shows a linear increase at low voltages (fit: $C=$ $\left.0.0205 V^{2}+954.8 ; R^{2}=0.95\right)$ and plateaus at high voltages (fit: $\left.C=0.0039 V^{2}+993.8 ; R^{2}=0.71\right)$. 


\subsubsection{Voltage to current output relationship}

A bilayer membrane is traditionally modelled as a capacitor and resistor in parallel [93]. Typical membrane resistance and capacitance values, however, produce an equivalent membrane impedance that is purely capacitive in the range of about $1-10,000 \mathrm{~Hz}$ [93, 77]. Since we only consider hair vibrations in the range of $100-2,000 \mathrm{~Hz}$, we can treat the membrane as simply a variable capacitor. The current through a capacitor is described as the time rate of change of capacitance times voltage:

$$
i=\frac{d}{d t}\left(V C_{B L}\right)
$$

Since we deal only with constant applied voltages in this study, we may consider $\frac{d V(t)}{d t}=0$. Modifying Eqn. 5.2 in this way, and combining with Eqn. 5.1, we obtain an expression for the membrane sensitivity to voltage, as derived by Sarles and Leo [61].

$$
i(t)=\frac{d C_{B L}(t)}{d t}\left[V+\alpha V^{3}\right]
$$

The term $\frac{d C_{B L}(t)}{d t}$ can be seen as the current contribution due to membrane vibration while $\left[V+\alpha V^{3}\right]$ as a gain due to voltage effects. Figure 5.2 shows how the power spectral density

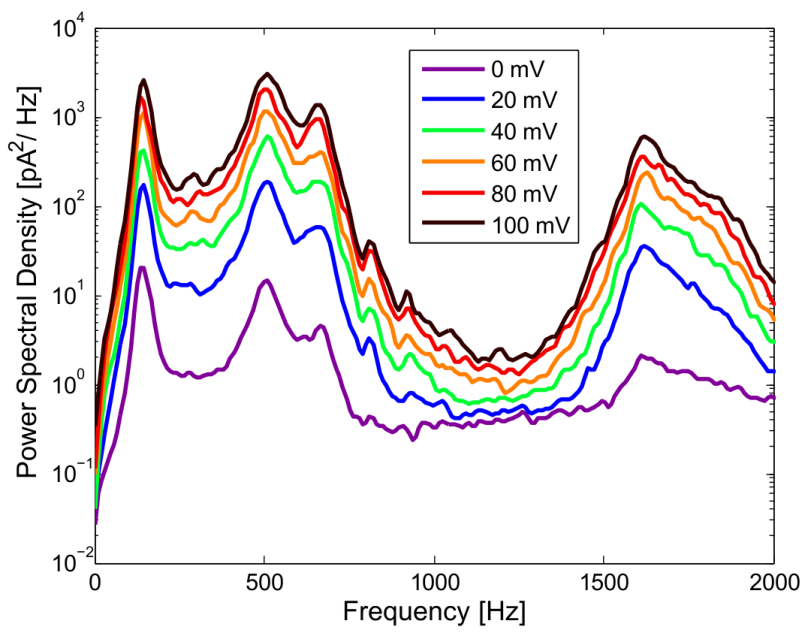

Figure 5.2: Power spectral density of the AHC output current at various voltages (colored lines) for a constant air flow. The PSD was computed using Welch's method. 
(PSD) of the AHC varies with voltage under a constant air flow. We notice that the PSD scales uniformly and frequencies do not shift with voltage. This confirms the assumption that voltage effects only serve to amplify the signal.

Exploring this further, we calculate how the magnitude of each frequency peak changes with voltage. We do this by integrating a region of the PSD $( \pm 80 \mathrm{~Hz})$ around each natural frequency. We then take the square root of this number to arrive at the amplitude spectral density (ASD), a measure analogous to the root mean square of the time signal. An advantage of the ASD is that we can isolate the current contributions of specific frequency ranges. Figure 5.3 plots the AHC ASD current versus applied voltage for regions around each natural frequency as well as the entire spectrum. It is important to note that these are not transfer functions, but rather measures of the sensor current output under a constant airflow. Fitting Eqn. 5.3 to full spectrum data shows excellent agreement $\left(R^{2} \approx 1\right)$, but a low value of $\alpha=8.2 V^{-2}$ leads us to conclude that electrowetting effects are hardly present in these tests. In fact, a linear fit $(\alpha=0)$ of the data is also in excellent agreement $\left(R_{\text {Linear }}^{2} \approx 1\right)$. For a linear fit, the capacitive rate of change $\left(\frac{d C}{d t}\right)_{A S D}$ (the slope of the line) is approximately $2,680 \mathrm{pF} / \mathrm{s}$. This value is significantly higher than values of $50-100 \mathrm{pF} / \mathrm{s}$ obtain in a previous

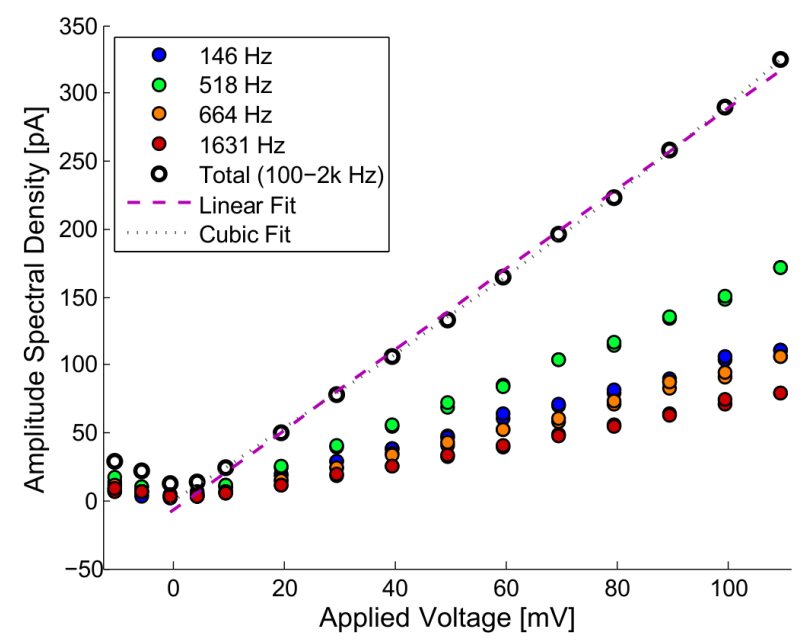

Figure 5.3: Amplitude current density of the AHC versus applied voltage for a constant air flow. Each ASD value is the square root of the PSD integrated over a region $\pm 80 \mathrm{~Hz}$ around each frequency. Colored points represent the measured natural frequencies. White dots represent the ASD for an integration over the entire spectrum $(100-2 \mathrm{k} \mathrm{Hz})$ (Total Spectrum Fit: $i_{A S D}=$ $2.2 \times 10^{-5} V^{3}+2.7 V ; R^{2} \approx 1 ; R_{\text {Linear }}^{2} \approx 1$ ) 
study [61].

Additional fits of Eqn. (5.3) for individual frequencies (Table 5.1) reveal low $\alpha$ values (4.9-12.1 $\mathrm{V}^{-2}$ ). Such strong linearity of frequency contributions is another good indication that applied voltage only amplifies the capacitive change of the bilayer. Estimates of the capacitance change in Table 5.1 show that mode 2 is the largest contributor to current amplitude, just as was seen in pulse characterization experiment.

Table 5.1: Eqn. 5.3 fit parameters for voltage sensitivity of each natural frequency.

\begin{tabular}{|c|c|c|c|}
\hline Mode & $\alpha$ & $\left(\frac{d C_{B L}}{d t}\right)_{A S D}$ & $\mathrm{R}^{2}$ \\
\hline $146 \mathrm{~Hz}$ & $4.9 \mathrm{~V}^{-2}$ & $964 \mathrm{pF} / \mathrm{s}$ & $\sim 1$ \\
\hline $518 \mathrm{~Hz}$ & $8.2 \mathrm{~V}^{-2}$ & $1438 \mathrm{pF} / \mathrm{s}$ & $\sim 1$ \\
\hline $664 \mathrm{~Hz}$ & $12.1 \mathrm{~V}^{-2}$ & $862 \mathrm{pF} / \mathrm{s}$ & $\sim 1$ \\
\hline $1631 \mathrm{~Hz}$ & $9.2 \mathrm{~V}^{-2}$ & $671 \mathrm{pF} / \mathrm{s}$ & $\sim 1$ \\
\hline
\end{tabular}

\subsection{Sensitivity to Airflow}

\subsubsection{Current output versus airflow magnitude}

To examine the sensor's sensitivity response to air flow, we excited the hair with constant air flows from 0-72 m/s, seen in Figure 5.4. The AHC was held at $50 \mathrm{mV}$ applied voltage throughout. The PSD shows an increase current amplitude with increasing air velocity. Predicted modes from stiffness values estimated experimentally in the characterization tests (black lines, $k=19 \mathrm{~N} / \mathrm{m}$ and $\tau=0.0011 \mathrm{Nm} / \mathrm{rad}$ ) show that the membrane is vibrating near the expected hair natural frequencies. The modal peaks do not shift, that is, the hair oscillation frequency is not a function of airflow velocity. Such behavior suggest that the air flow hair around the hair causes a state of free vibration and does not significantly alter its dynamics within the range studied here. Reynolds number calculations $\left(10^{4}-3 \times 10^{5}\right)$ for the airflow indicate turbulence could be source of the free vibration. Laminar flow has been shown only to deflect the hair, but not to vibrate it 83. Figure 5.5 illustrates how the ASD changes with air speed for individual frequencies and the total spectrum. A linear fit of the total spectrum values shows excellent agreement $\left(R^{2} \approx 1\right)$ and yields an approximate air velocity sensitivity (slope of the purple fit line) to be $3.39 \mathrm{pA} / \mathrm{m} \mathrm{s}^{-1}$. Linear sensitivity to 


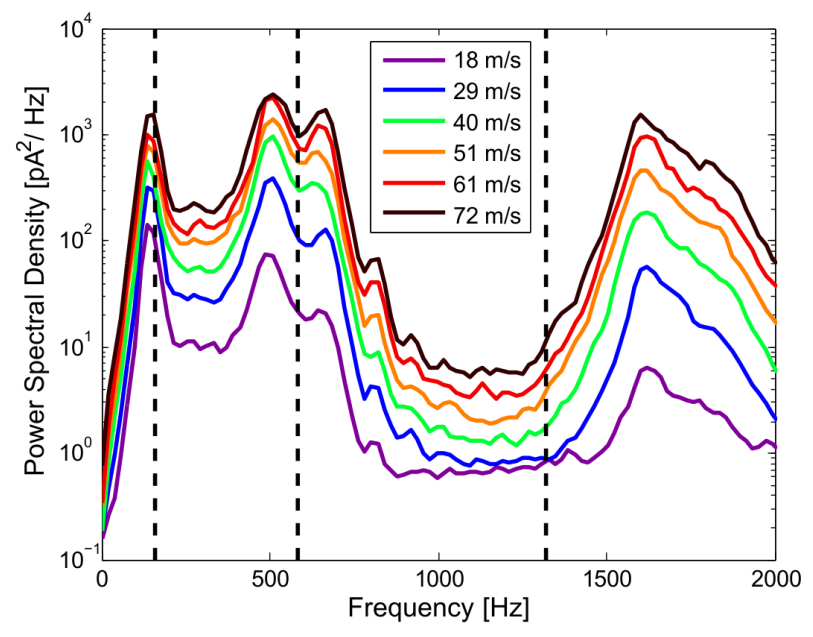

Figure 5.4: Power spectral density of the AHC output current at various air flow (colored lines) for a constant $50 \mathrm{mV}$ applied voltage. The black lines show predicted natural frequencies for $k=19$ $\mathrm{N} / \mathrm{m}$ and $\tau=0.0011 \mathrm{Nm} / \mathrm{rad}$. The PSD was computed using Welch's method.

velocity was also shown by Sarles and Leo 61]. ASD traces of individual frequencies also show excellent linearity with sensitivities ranging from $1.06 \mathrm{pA} / \mathrm{m} \mathrm{s}^{-1}$ for the $146 \mathrm{~Hz}$ mode to $2.08 \mathrm{pA} / \mathrm{m} \mathrm{s}^{-1}$ for the $518 \mathrm{~Hz}$ mode. Higher order fits of these traces also show slight decreases in sensitivity for low frequency modes $(146$ and $518 \mathrm{~Hz})$ and slight increases for high frequency modes $(664$ and $1631 \mathrm{~Hz})$. This behavior can be observed visually at high air speeds in Figure 5.5, where some modes begin to curve downward and others upward. This may indicate some slight redistribution of energy from low to high modes as velocity increases. The overall sensitivity of the sensor, however, remains quite linear. 


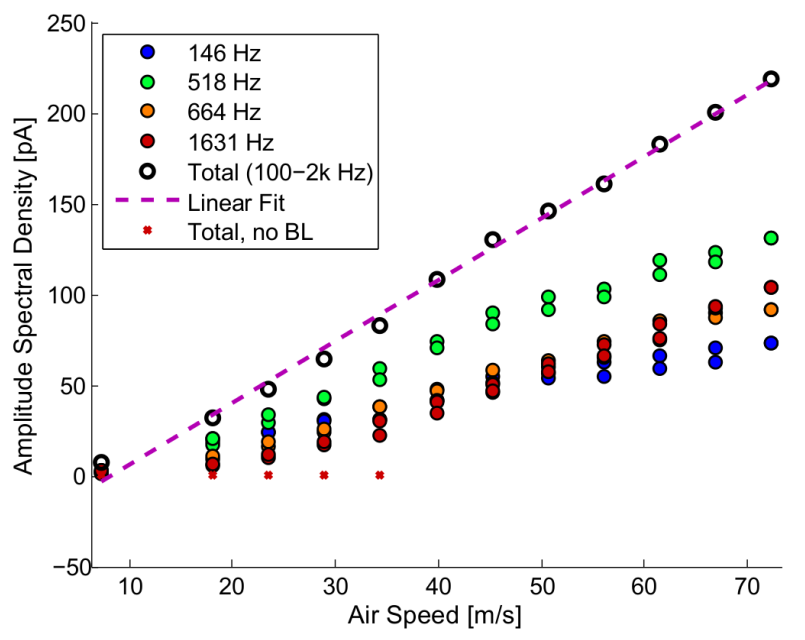

Figure 5.5: Relation between the spectral amplitude and air speed at $50 \mathrm{mV}$ applied potential. Modal frequency amplitudes are shown in color and the total integrated spectrum is shown in white. (fit: $i_{A S D}=-27.5+3.39 A S ; R^{2} \approx 1$ ) 


\section{Chapter 6}

\section{Flow Localization using Arrays of AHCs}

Understanding the electrical and dynamic behavior of AHC units paves the way for creating array structures which could significantly improve sensing capability. Inadequate substrate designs and issues with bilayer durability had made creating such arrays difficult until now. To prove that this concept could be achieved with the AHC design presented in this work, we assembled linear arrays of up to three units and used them to sense the lateral position of an air source. A significant challenge in processing the sensors' output was the limitation of one input channel on the Axopatch 200B measurement unit. To overcome this, the author created a method entitled Hair Frequency Response Decomposition (HFRD) that separates a combined signal back to its constituent parts based on the signal frequency content. In this chapter, we explain the HFRD method and show how it was used in combination with AHC arrays to interpret air flow information and assess AHC array sensing capabilities.

\subsection{AHC Array Measurement}

In order to validate the membrane-based AHC array concept and assess its sensing capabilities, we tested an array of three AHCs with an airflow of varying position and intensity (Figure 6.1). Each AHC was fabricated with a hair of different length $(33 \mathrm{~mm}, 26 \mathrm{~mm}$, and $20 \mathrm{~mm}$ ), giving it a unique vibrational response. In each test, a constant-velocity air source was moved incrementally across the AHC array, as shown in the figure. The air nozzle 


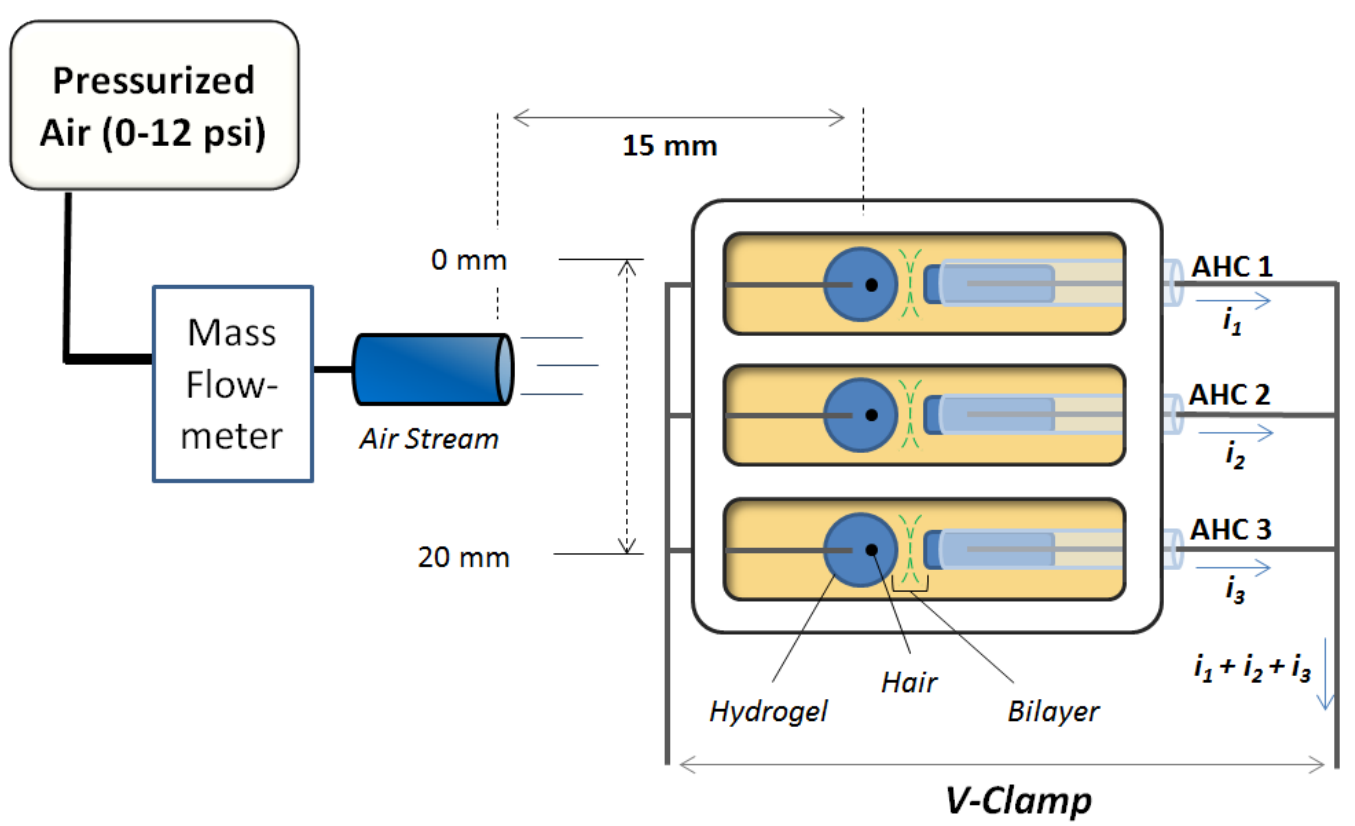

Figure 6.1: The test array consisted of three adjacent AHCs of different hair lengths: $33 \mathrm{~mm}$ (AHC 1), $26 \mathrm{~mm}$ (AHC 2), and $20 \mathrm{~mm}$ (AHC 3). The array was excited by sweeping an air source laterally in $2 \mathrm{~mm}$ intervals. The $0 \mathrm{~mm}$ position was slightly to the outside of AHC 1 while the final position was slightly to the outside of AHC 3, a span of $20 \mathrm{~mm}$. The air source was placed at a distance of $15 \mathrm{~mm}$ from the AHC hairs and wide enough to cover a lateral space of approximately $10 \mathrm{~mm}$, enough to excite two hairs at once. The airflow rate was measured through a mass flowmeter and the summed current output was measured through a voltage clamped, parallel circuit configuration.

was fixed at each position approximately 5-10 seconds while the AHC output response was collected. The air profile was wide enough to excite multiple hairs at once. The measured output current was the combination of the individual AHC currents, which were wired in parallel. Five of these position sweep experiments were conducted, each with the same hair lengths but different bilayers.

As the airflow moved, the excitation levels at each position were calculated via the Hair Frequency Response Decomposition method, therefore mapping the frequency and magnitude output of the AHC array to the location of the air flow. The method is explained in detail the following section. The outcomes of this procedure allow us to first evaluate how well the AHCs communicate airflow information simultaneously, that is, how reliable the sensors' output frequency and magnitude are. Electrically joining these sensors, for example, could 
potentially affect unit transduction properties and stability. In addition, we can analyze how the AHC array geometry plays are role in interference and spacial resolution. Finally, we can assess the limits of the HFRD method itself. For example, how robust is it to deviation from the assumed behavior? Under what conditions would it be applicable to larger arrays? Answers to these important questions can direct the development of larger, more complex membrane based AHC arrays.

\subsection{Hair Frequency Response Decomposition}

The hair frequency response decomposition method (or HFRD) is based on the principle of modal superposition, which states that a complex frequency response is the linear combination of individual modes [98]. By this logic, the output response of a single AHC can be described as a sum of various modal contributions. Furthermore, according to Kirchoff's current law, the total AHC array output current is a superposition of individual unit output currents [99]. When each hair in an AHC array is of a different length, each vibrational response will differ significantly. In this case, the output of multiple AHC sensors vibrating concurrently could be decomposed into the unique spectral responses of the corresponding hairs. This provides a way to measure the outputs of multiple sensors through a single output channel.

The method was partly inspired by the biological function of the basilar membrane, which performs a similar but inverse signal processing operation. The basilar membrane is mechanically tuned such that its location of resonance is a function of the input frequency [3]. Thus, through an array of biological hair cells that line the membrane from end to end, the brain can map an excitation at a specific location to a particular sound frequency. The arrays in this study, on the other hand, have a unique frequency response associated with each location. This allows us to map a set of frequencies to a particular location. While the HFRD method cannot match the accuracy of multiple measurement channels, it is quite useful when these are limited as in this study.

The HFRD method makes three assumptions about the AHC output behavior based on experimental observations from Chapters 4 and 5. The first is that, within the experimental time-frame (15-20 mins), the bilayers undergo negligible changes in area. In other words, each AHC capacitance is assumed to stay constant throughout testing. This is based on the ob- 
servation of stable bilayer capacitances for over 30 minutes after formation (see Section 2.2). However, the capacitances are not necessarily assumed to be equal, and thus current output magnitudes are allowed to vary from AHC to AHC. The second assumption follows from the results from Figure 5.4 and holds that the modes of each AHC output response will scale uniformly with changing airflow. The output power spectral densities (PSDs) of a particular AHC are thus considered to be scalar multiples of each other within the testing range of 20 to $120 \mathrm{~m} / \mathrm{s}$. It was also shown that the integrated amplitude spectral density (square root of the integrated PSD) will scale linearly with airflow velocity. The HFRD method uses this fact to estimate the flow velocity magnitudes each hair is experiencing. Finally, the third assumption holds that output current of the array circuit is linear superposition of the individual currents of each AHC unit $\left(i_{1}+i_{2}+i_{3}\right.$ in Figure 6.1), as given by Kirchoff's current law 99 .

The process of hair frequency signature decomposition begins by taking reference PSD measurements of each AHC unit independently in standard input conditions: air flow rates at 10 and 20 SLPM with the air nozzle centered on the hair, and $-20 \mathrm{mV}$ applied voltage. The applied voltage was chosen to provide a balance between increased sensitivity and added electrical stress on the bilayer. The purpose of these tests was to observe the decoupled responses of the AHCs. A small five second sample of the vibrational response was taken and subsequently converted to a power spectral density (PSD). In order for the HFRD method to work effectively, each PSD response must be uniquely distinguishable from the rest, hence the differing hair lengths.

A peak fit of the numerical PSD data utilized the superposition principle to provide a mathematical description of the individual spectral response of each AHC, as shown in Figure 6.2. This fit is referred to as the "reference fit" $R F_{h}(f)$ and was created from a sum of Lorentzian functions as in equation 6.1. where each sum term described a single mode of the output response. Based on the second assumption above, we expect a scalar multiple of the reference fit to adequately represent all possible outputs for a particular AHC unit. The Lorentzian functional form was chosen because it provides an accurate mathematical description of the modal peak shapes. Other types of peak-shaped functions, such as Gaussian distributions, can also be used effectively. 


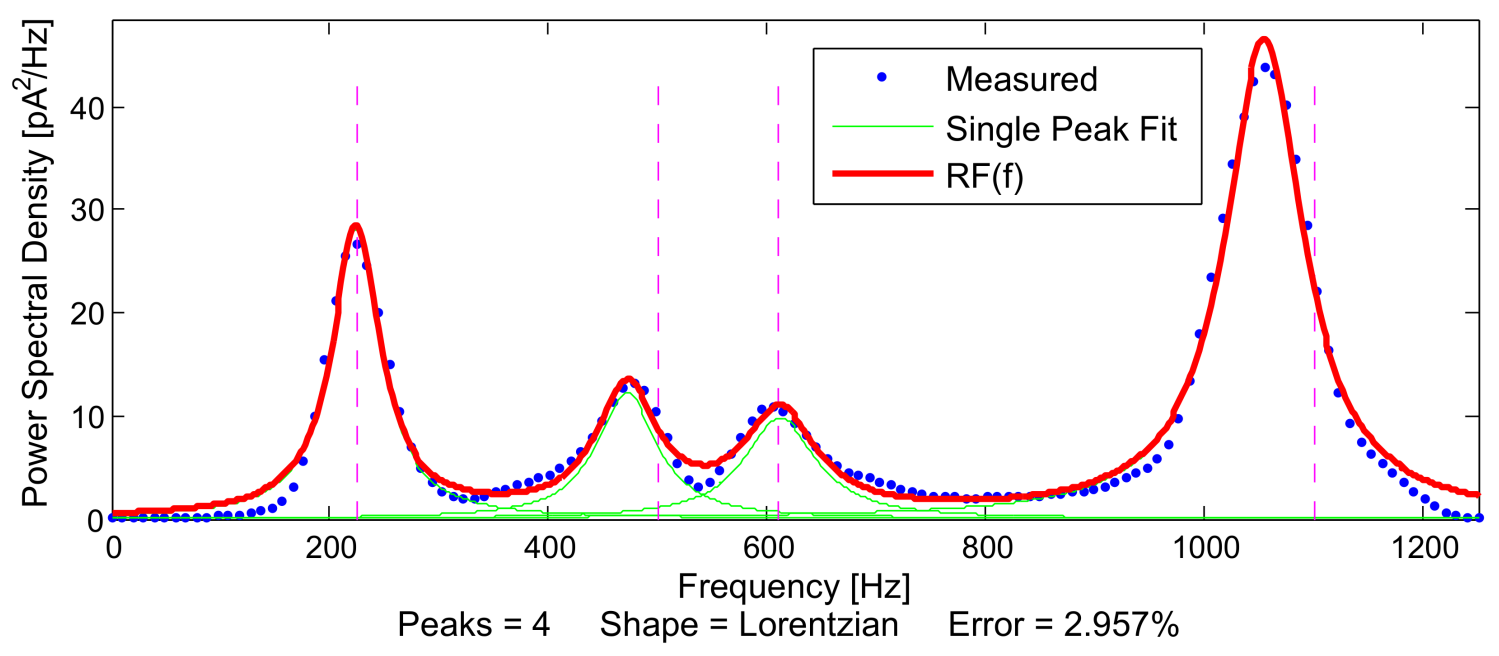

Figure 6.2: Reference fitting operation for a sample power spectral density of an individual AHC. The blue dots are the experimentally measured PSD values of 5-10 second time signal. The four green lines are the individual peak fits described by a single summation term in equation 6.1 . The red line is the sum of the four green lines, i.e. $R F_{h}(f)$. Note that the average mean squared error in this example is about $3 \%$.

$$
R F_{h}(f)=\sum_{n=1}^{N} \frac{A_{n}}{\left[1+\left(\frac{f-f_{n}}{w_{n}}\right)^{2}\right]}
$$

The independent variable $f$ is the PSD frequency, while subscripts $h$ and $n$ denote the AHC number and peak number respectively. $H$ represents the total number of AHC units and $N$ the total number of peaks used for the fit. In this work, $H=3$ and $N=4$, as three AHCs were used and up to four modal peaks were observed in certain responses. $A_{n}$ is the peak height parameter, $f_{n}$ is the peak frequency and $w_{n}$ is the peak's half-width at half-height.

The next step was to evaluate the fully connected array output, as shown previously in Figure 6.1. This is done by calculating the linear combination of reference fits that best matches the output at a specific air position, in order to determine which AHCs are contributing to the response at that position. The calculation is shown in Eq. 6.2 and referred to as the "combined fit" $C F(\mathbf{c}, f)$. It is a sum of the unique reference fits $R F_{h}$, each weighted by a contribution coefficient $c_{h}$. This function follows directly from the third assumption above 
and can be fitted to the total AHC array output through the contribution coefficients $c_{h}$, represented by the vector $\mathbf{c}=\left[\begin{array}{lll}c_{1} & c_{2} & c_{3}\end{array}\right]$ in the case of three AHCs. The fit is performed using an iterative non-linear algorithm so that combination of coefficients minimizes the least squared residuals of the fitting function [100, 101]. An example of this operation is shown in Figure 6.3 and the programmatical implementation can be found in Appendix C. The contribution coefficients can be used to determine the relative excitations of the three AHCs in the array, which can be correlated to the horizontal location of the airflow field.

$$
C F_{h}(\mathbf{c}, f)=\sum_{h=1}^{H} c_{h} * R F_{h}(f)
$$

The contribution coefficients $c_{h}$ are unitless scalars which represent the fraction of excitation an AHC is receiving, relative to the reference PSD curve. The $c_{h}$ value of a particular AHC signal will be approximately one when the airflow is centered on the AHC and of the same magnitude as the reference test. The square root of a signal's $c_{h}$ value times its integrated reference PSD is approximately equal to the amplitude spectral density and RMS currents

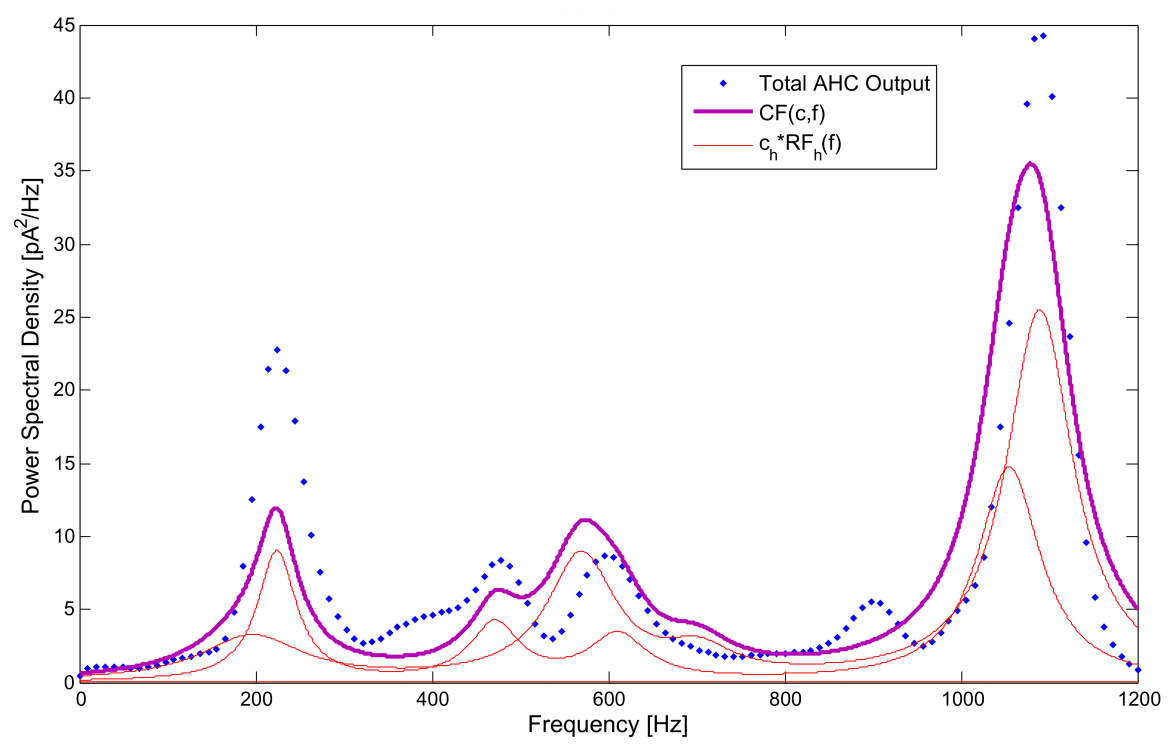

Figure 6.3: Combined fitting operation for a sample power spectral density of the simultaneous output of three AHCs (blue dots). The three red lines are the weighted reference fits for each AHC, that is the function $c_{i} * R F_{i}(f)$ for AHC $i=1,2,3$. The violet line is the sum of the three red lines, i.e. $C F(c, f)$ from equation 6.2. Note that one red line is very close to zero, suggesting that the particular reference fit contributed little to the total output. 
of the same signal (eq. 6.3). Figure 6.4 shows the application an HFRD analysis to the same data from section 5.2. The HFRD estimates of the RMS current match closely with the ASD of the signal. The results also show that the HFRD estimates scale linearly with the airflow velocity, just as the ASD, validating the second assumption above. This linear sensitivity to airflow velocity allows the use of just two reference fits to interpolate airflow velocity from a contribution coefficient. The sensitivity characteristics of the AHC are covered in detail in the next section.

$$
\sqrt{c_{h} \int P S D_{r e f, h}} \approx \int A S D_{h} \approx R M S_{h}
$$

To clarify the entire process, Figure 6.5 shows a visualization of how the reference and combined fits are used together. The HFRD process must be performed at each air position and needs at least 1 second of data to provide accurate results. An example test is shown

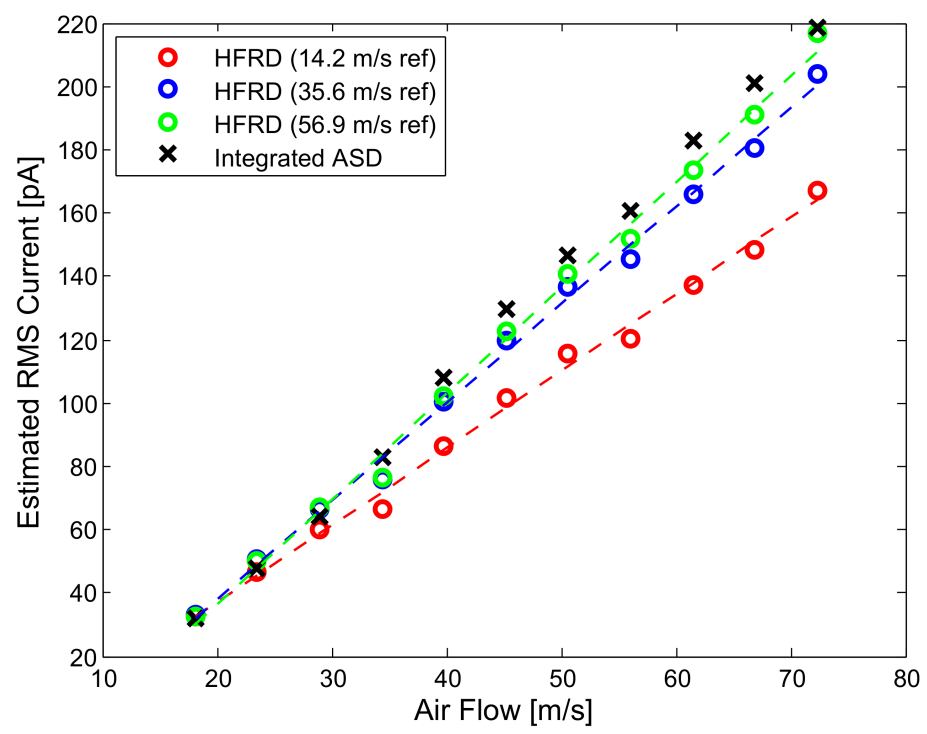

Figure 6.4: The HFRD method is applied to the airflow response data from section 5.2, By selfreferencing the method to PSD data at different airflow velocities $(14.2,35.6$, and $56.9 \mathrm{~m} / \mathrm{s})$, we can create a curve of contribution coefficients. The coefficients $c_{h}$ are then scaled as in equation 6.4, to convert them to RMS estimates (points in red, blue and green). Notice how the points agree well with the integrated ASD of the same data, another measure of RMS. Also notice the high linearity of the HFRD estimates, shown by the dotted linear fits (red: $R^{2}=0.995$, blue: $R^{2}=0.996$, green: $R^{2}=0.996$ 
in the figure, with the airflow at a position $8 \mathrm{~mm}$ from the starting point, centered between AHC 1 and 2. Note how the weighted reference fits $\left(c_{h} * R F_{h}\right)$ are unique and used to estimate the current response of each AHC. Recall that these must be acquired and stored through a separate test in which each AHC is tested independently (not shown in the figure). The best linear combination that matches the PSD of the measured data ("actual output") yields the combined fit $C F(c, f)$. The parameters of interest, however, are the contributions coefficients $c_{h}$ which can be used to determine the level of excitation each AHC is experiencing.

In certain cases, large differences in the maximum amplitudes of the reference fits occurred, typically when AHCs had sizeable differences in their current output RMS levels. For these situations, the fitting algorithm for the combined fits tended to bias the contribution coefficient solution toward the references at smaller levels, misrepresenting the dominant dynamics. To correct this bias, the author developed a data filter that adjusted the coefficient values based on their normalized contributions, that is when all references fits were of equal

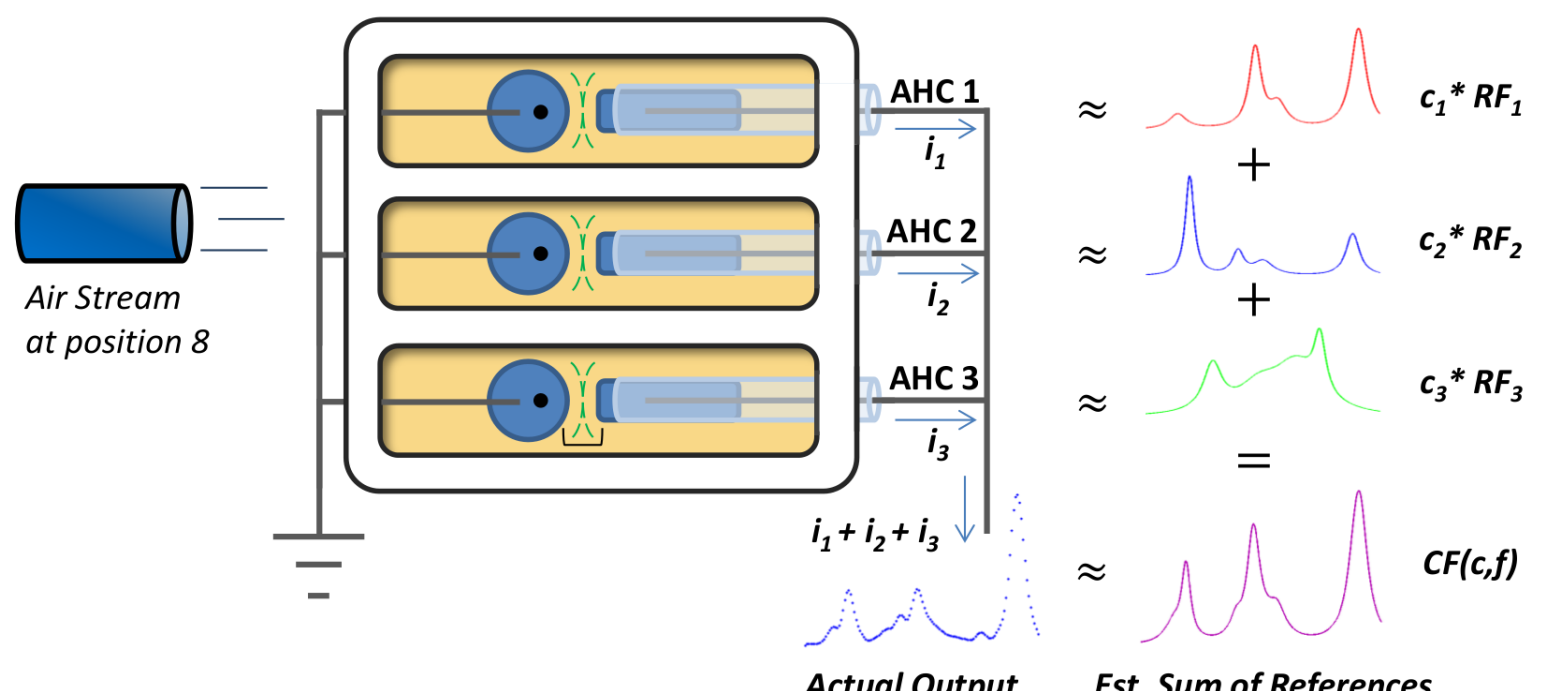

Actual Output

Est. Sum of References

Figure 6.5: Visualization of an HFRD calculation at a measurement instance. The flow is fixed at a position $8 \mathrm{~mm}$ from the starting point. The actual output shown is the PSD of the measured time signal, assumed to be the sum of individual AHCs $\left(i_{1}+i_{2}+i_{3}\right)$. Each current is approximated by its reference fit $R F_{h}$, calculated before the test (see Figure 6.2). The reference fits are scaled by the contribution coefficients $\left(c_{h}\right)$, which represent the relative level of excitation that each AHC is receiving at the particular airflow location. The sum of the terms (see Eq. 6.2) results in the combined fit $C F(c, f)$ which is an approximation of the actual output. The precise values of $c_{1}, c_{2}, c_{3}$ are chosen by a fitting algorithms which minimizes the error between $C F(c, f)$ and the actual output. 
amplitude. This required $c_{\text {norm }}$, an additional contribution coefficient calculation (Eq. 6.1) where all the reference fits (Eq.6.2) were normalized to their maximum peak amplitude. The mathematical implementation of the filter is shown in Eq. 6.4. The final, adjusted coefficient values $c_{\text {adjust }}$ are generated my multiplying the original values by the filter coefficients $f_{h}$.

$$
\begin{array}{r}
f_{h}=\frac{c_{\text {norm }, h}}{\sum_{i=1}^{H} c_{\text {norm }, i}} \\
c_{\text {adjust }, h}=c_{h} f_{h}
\end{array}
$$

\subsubsection{Air Velocity Profile}

Before discussing the AHC response to an applied airflow, it is important to understand the characteristics of the flow itself. In all array experiments, the air source was a circular tube of $3.2 \mathrm{~mm}$ inner diameter, positioned $15 \mathrm{~mm}$ axially from the AHC array (see Fig. 6.1). The Goertler solution for a free turbulent jet exiting a circular nozzle shows the radial air velocity distribution $u(r, x)$ to be:

$$
\begin{gathered}
u(r, x)=\frac{u_{m}}{1+0.125(\sigma r / x)^{2}} \\
u_{m} \quad=\frac{U_{0} C_{1}}{x / r_{0}}
\end{gathered}
$$

where $r$ is the radial distance from the nozzle's center, $x$ is the axial distance from the nozzle, $u_{m}$ is the maximum velocity, $U_{0}$ is the uniform velocity at $x=0$ and $r_{0}$ is the nozzle diameter. The constants $\sigma$ and $C_{1}$ are derived experimentally and are approximately equal to 18.5 and 5.75, respectively [102]. Equation 6.6 shows that the air velocity distribution is bell-shaped with a maximum velocity occurring at $r=0$. The value of the maximum velocity $\left(u_{m}\right)$ itself can be estimated from Equation 6.7 using the measured value of airflow rate $Q$ through the tube, since $U_{0}=Q / \pi r_{0}^{2}$. 


\subsubsection{Reference Signals and Sensitivity Analysis}

Following the procedure of the HFRD method, each array experiment began by measuring the reference responses of each AHC unit, that is, the output when isolated from the rest of the array. This was done after all bilayers were formed by individually probing each AHC and measuring the output response to a centered air stream at two prescribed airflow rates: 10 and 20 SLPM. The air velocity experienced by the hair in each case was estimated using equation $6.7(12.7$ and $25.4 \mathrm{~m} / \mathrm{s})$. We know from the results shown in Figure 6.4 that $\sqrt{c_{h}}$ scales linearly with airflow. Thus, the two reference measurements could be used to estimate the corresponding sensitivity curves using similarly calculated HFRD $c_{h}$ values. Assuming that the sensor output is zero at zero airflow, we relate the HFRD output to the velocity $u$ at the $\mathrm{AHC}$ as

$$
u=\frac{1}{\beta_{h}} \sqrt{c_{h}}
$$

were $\beta_{h}$ is the HFRD sensitivity to air velocity, calculated experimentally from a linear fit of the reference measurements as shown in Figure 6.6.

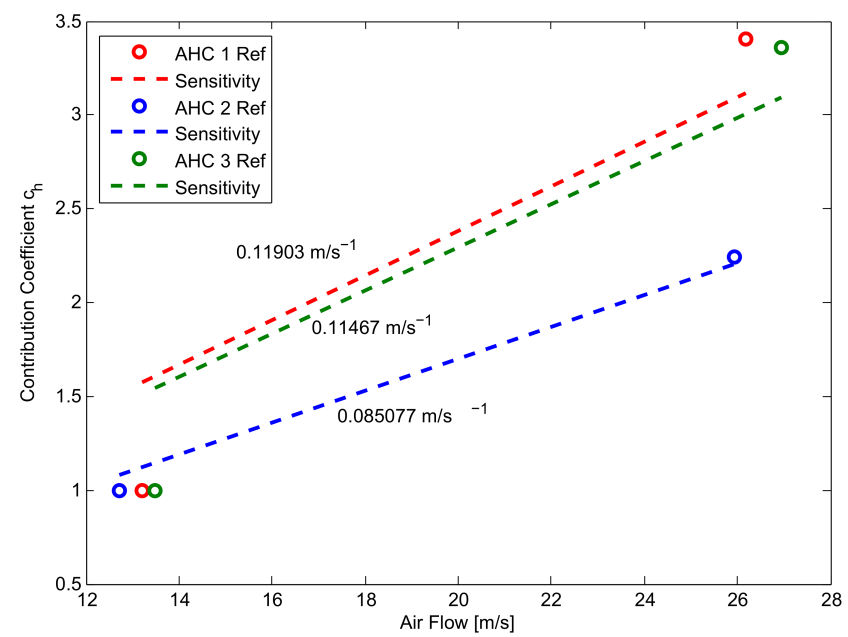

Figure 6.6: Sensitivity of the $c_{h}$ coefficients to applied air velocity for an array of three AHCs. The circular data points indicate the calculated reference values at approximately 12.7 and 25.4 $\mathrm{m} / \mathrm{s}$. The dotted lines indicate the estimated linear sensitivity curve from a fit of equation 6.8 . The $\beta_{h}$ sensitivity values (slope of the line) are labelled next to each curve: $\beta_{1}=0.119 \mathrm{~m} / \mathrm{s}^{-1}$, $\beta_{2}=0.085 \mathrm{~m} / \mathrm{s}^{-1}$, and $\beta_{3}=0.115 \mathrm{~m} / \mathrm{s}^{-1}$. 
A series of four separate array tests, using the same hairs but new bilayer formations each time, were used to study the variations in AHC sensitivity to air flow. The results, shown in Figure 6.7, suggest that the value of the $b_{h}$ sensitivity is dependent on both the length of the hair as well as the bilayer size. Nominal airflow sensitivity measurements of AHC reference data show that the average sensitivity increases with hair length. The variability, however, indicated no significant difference between the two lowest lengths. Normalizing the nominal data by the bilayer capacitance had the effect of reducing the standard deviation, with one exception, suggesting that bilayer area also has an effect on sensitivity.

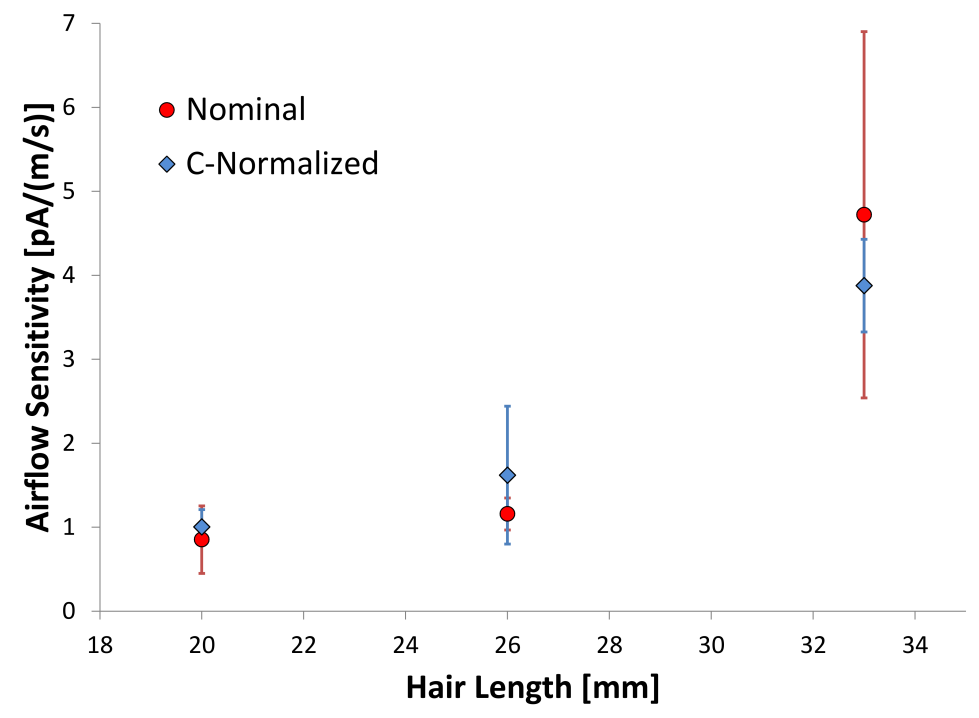

Figure 6.7: Nominal airflow sensitivity measurements of AHC reference data (red circles) show that the average sensitivity increases with hair length. Standard deviation error bars indicate no significant difference between the two lowest length. Normalizing the nominal data the bilayer capacitance tended to reduce the standard deviation, except for the $26 \mathrm{~mm}$ hair, suggesting that bilayer area also has an effect on sensitivity.

\subsubsection{Flow Position \& Magnitude Mapping via HFRD}

Converting the raw sensor data into flow position and magnitude informations takes place as four-step process, outlined in Figure 6.8. For the air nozzle at a fixed position, the PSD of the output signal is processed using the HFRD method to calculate the superposition of reference signals that will result in the best fit (Figure 6.8A). It is important to note that experimental variation can cause discrepancies in the fitting operation. Changes in 
the bilayer area, droplet contact, and exact incident angle of the flow can all change to a degree the frequency and magnitude of the output modes. As such, we observe that while some modes are fitted well (e.g. the first mode), others are quite poor and often attempts by the solver to compensate for deviations from the reference behavior. Nevertheless, the superposition coefficients $\left(c_{h}\right)$ resulting from this process can be mapped to the corresponding AHC positions to indicate the levels of excitation each one is experiencing, relative to the reference data (Figure 6.8 $\mathrm{B}$ ). In the example, the $c_{2}$ value of 2.8 indicates that the excitation magnitude at AHC 2 is 2.8 times that of the reference signal measured at $10 \mathrm{~m} / \mathrm{s}$ airflow. Using two reference data sets per AHC and the relationship described by Equation 6.8, we generate sensitivity curves to interpolate the $c_{h}$ values to an actual air velocity estimate (Figure 6.8C). In the case of $\beta_{2}$, for example, it indicates how much a 2.8 -fold increase in output correlates to air velocity. Applying this conversion to the results in Figure 6.8B produces an estimated air velocity profile for the air at that position (Figure 6.8D). Since the air position $(8 \mathrm{~mm})$ in the example corresponds to slightly left of the position of AHC 2 , it makes sense to see an estimated profile that is highest at AHC 2 but skewed toward AHC 1. For the arrays in this study, the spacing between hairs was approximately $8 \mathrm{~mm}$, resulting a profile resolution of the same value. More AHC's in a denser pattern could create a much more detailed estimate. 

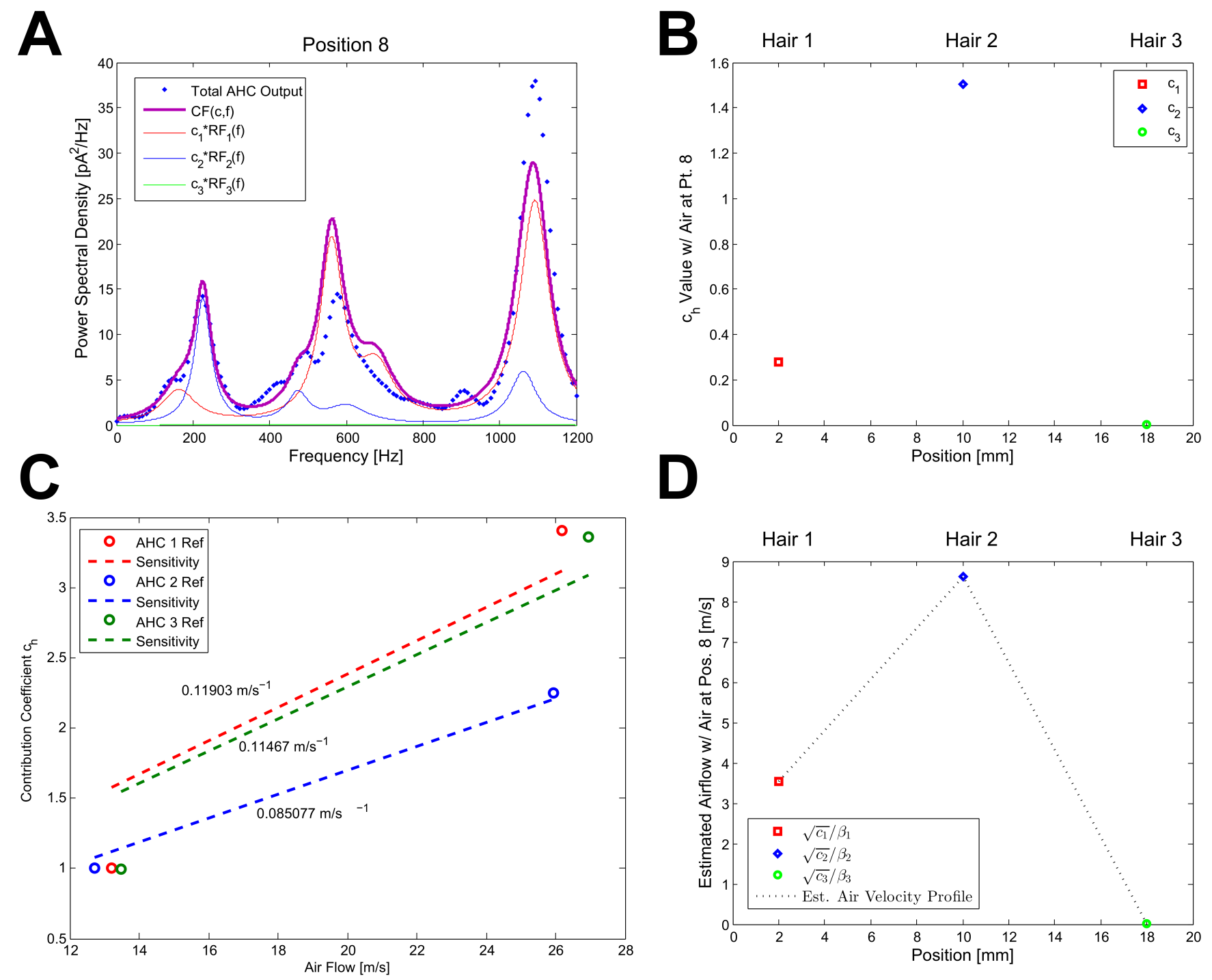

Figure 6.8: Illustration of the airflow mapping process using HFRD for a flow postioned at 8 $\mathrm{mm}$ with a magnitude of $19.32 \mathrm{~m} / \mathrm{s}$. A) The PSD of sensor data (blue dots), with the air source position on $8 \mathrm{~mm}$, is fitted using the HFRD method to obtain the $c_{h}$ coefficient values. B) The $c_{h}$ values are mapped to their respective positions, indicating the relative excitations of the AHC sensors. C) Sensitivity estimates for each of the three AHC provide a way to convert the $c_{h}$ values to an air velocity reading. D) $c_{h}$ values, converted to velocity estimates using the sensitivity curves from (C), have been mapped once again to their spacial locations. This provides a visualization of the estimated velocity profile for an air source positioned at $8 \mathrm{~mm}$. 
Applying the process above continuously as the air source is moved across the array in 2 mm increments creates a total mapping of the AHC output to position, shown in Figure 6.9. We observe that each AHC output peaks when the air excitation was centered on its hair location and drops gradually away to zero as the excitation distance becomes greater. The overall clarity of the peaks and smoothness of the curves validates the capability of the AHC array system to interpret the air flow's position. The frequency content of the array output is clear enough for the HFRD to reliably separate the component signals. One reason for this is that membrane vibrations are well isolated from noise and the mechanical vibrations of other hairs. Another reason is that the bilayer lens size or contact area (i.e. capacitance) did not significantly influence the dynamics of the output. The only changes caused specifically by bilayer properties were changes in the output magnitude; the relative modal values, however, remained consistent. When irregularities in the position mapping did occur, as with the AHC 3 signal at $8 \mathrm{~mm}$ in Fig. 6.9, they were typically near the noise floor and/or in the presence of other dominant signals. In these cases, the algorithm was misled to attribute certain small signals to AHC dynamics.

We also note a finer resolution $(2 \mathrm{~mm})$ estimate of the air velocity profile at each AHC. The theoretical air velocity distributions are shown by the dotted lines, peaking at the maximum profile velocity (estimated by Equation 6.7 from the mass flowmeter measurement) and decreasing asymptotically to 0 with radial distance from the nozzle (Equation 6.6). We see that AHC 2 signal results in a close match with the theoretical curve, while the AHC 1 and 3 signals are clear under- and overestimates, respectively. In general, it was observed that the estimated peak air velocities, when compared the theoretical value, tended to vary by an average of $12.5 \mathrm{~m} / \mathrm{s}$. The discrepancies are attributed to deviations from the constant capacitance and uniform modal distribution assumptions of the HFRD method. Figure 6.10A shows an example of how the bilayer capacitance/area can before and after an airflow test (about 30 minutes long), causing significant changes in membrane sensitivity to airflow. Although bilayer membranes were observed to remain stable for these time frames in undisturbed conditions, continuous excitation and substrate manipulation may disturb the stability of the aqueous lipid lenses and cause a rearrangement of their original contact configuration. When these fluctuations occur between the times of a reference and a test measurement, they can skew the air velocity estimation. Figure 6.10B in turn shows how increasing air flow can change the relative modal values of the response, violating the uniform modes assumption. Notice how, when the air velocity is increased, there is not only an overall increase in energy (as expected) but also a significant redistribution of it from the first mode 


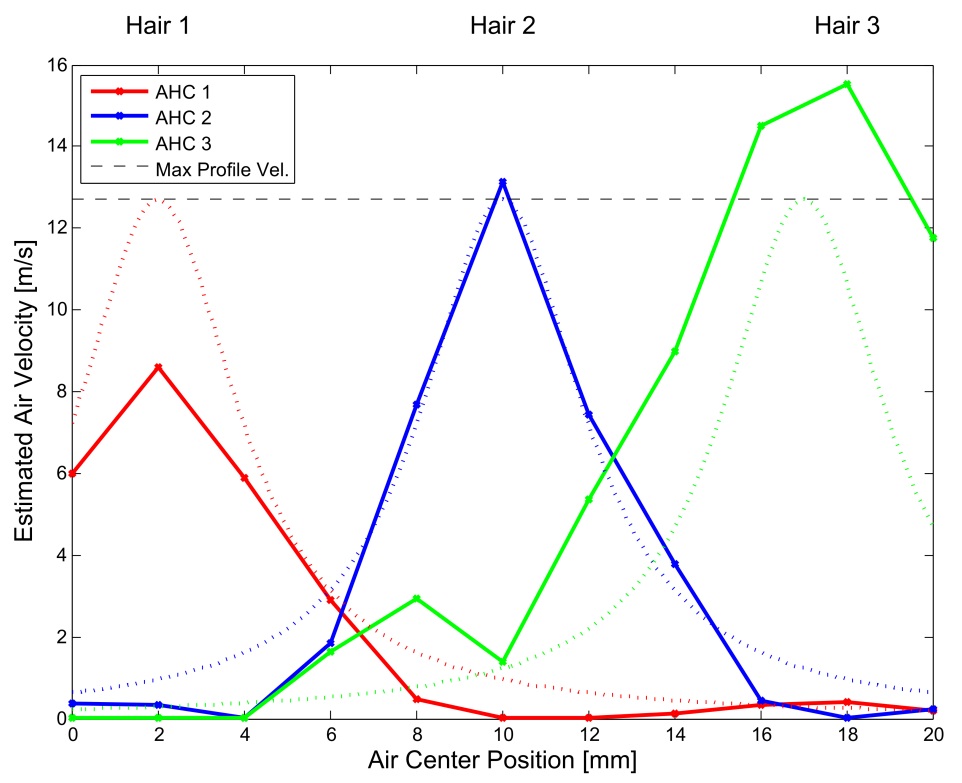

Figure 6.9: Hair frequency response decomposition of an array output signal shows the excitation levels of the three AHC components (red, blue and green curves) as an air source is moved incrementally across (step size of $2 \mathrm{~mm}$ ). The labels at the top of the graph indicate the position of each AHC hair (2, 10 and $18 \mathrm{~mm})$. The horizontal axis indicates the position of the center of the air nozzle, i.e. the position of maximum air velocity. The air flow profile in this case had a maximum velocity of $20.7 \mathrm{~m} / \mathrm{s}$, indicated by the dotted black line. The estimated air velocity values are based on linear interpolation of two reference points, and in ideal conditions should peak at the maximum applied velocity. The discrepancies observed here, particularly in AHCs 1 and 3, suggest instabilities in the sensitivities of the bilayer membranes.

to the fourth. This phenomenon can be attributed to changes in flow dynamics that excite higher modes as the air velocity increases and becomes more turbulent. It is seen as an issue solely dependent on the hair-flow interaction and not associated with the membrane. Nevertheless, the formulation of the HFRD method cannot adjust for this redistribution of modal energy, causing slight under or overestimates of the response.

Based on these results, we can make certain conclusions about the use of lipid bilayer membranes in AHC applications. Firstly, bilayer membranes produce clear and consistent transduction of the vibrational behavior of the hair. The dynamic distortion introduced by the membranes is small: the output frequency content is consistent even while the bilayer size or the bilayer itself change. This leads to AHCs that quite accurately map the signal content to the air excitation location. The single biggest obstacle to application is fluctuations in bilayer size while under continuous excitation, which causes changes in airflow sensitivity. This 

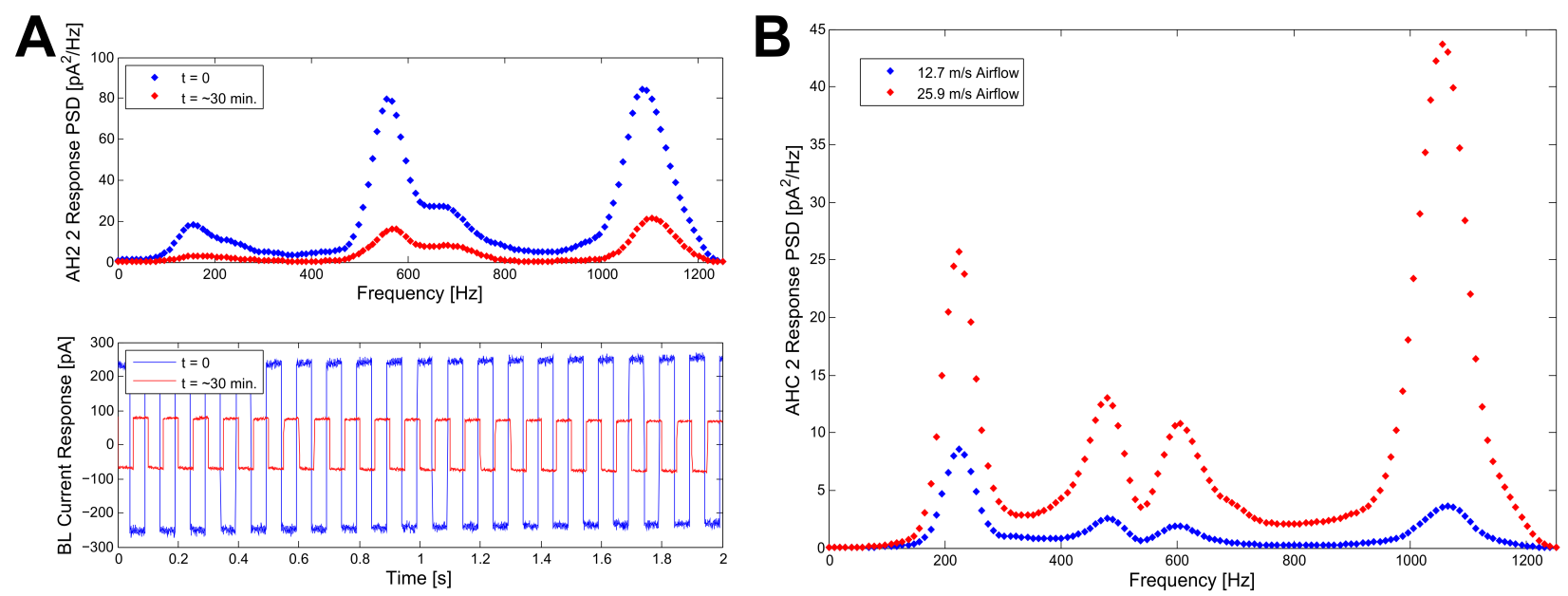

Figure 6.10: Two examples of experimental deviations from the simplifying assumptions of the HFRD method. A) The top figure shows two output PSD responses of AHC 2 at $12.7 \mathrm{~m} / \mathrm{s}$ with the same bilayer, taken before and after an airflow test, approximately 30 minutes apart. A magnitude decrease of $74 \%$ is observed. On the bottom, we see the respective bilayer triangle wave time responses, whose magnitudes are directly tied to the capacitance and area of the bilayer. A decrease of about $69 \%$ observed in this case indicates a similar decrease in bilayer size. B) The output PSD responses of two successive tests at 12.7 and $25.9 \mathrm{~m} / \mathrm{s}$ demonstrate a change in relative modal values, most noticeably between the first and fourth output modes.

phenomenon is generally impossible to separate from changes in the airflow magnitude itself, thus causing difficulties in the estimation of the excitation magnitude. Despite this, many bilayers demonstrated a general ability to withstand the dynamic fabrication and testing environment, which involved, droplet manipulations on the order of millimeters, translation of the substrate on the order of centimeters, and hair vibrations with millimeter scale amplitudes. It is nevertheless impractical to use the assembly methods presented here to create arrays of more than five AHCs, where hand fabrication provides insufficient precision and multiple-bilayer stabilization becomes increasingly difficult. With proper MEMs fabrication, however, lipid bilayer arrays could be extended to much greater numbers.

In design terms, we observe that the hairs provide measurements of a very narrow section of the flow field. This is a general advantage of hair cell sensors. We were able to spatially sample different points of the flow profile simultaneously by geometric arrangement of the AHC units. The array configuration tested here features $7 \mathrm{~mm}$ lateral resolution. Subsequent versions of this systems could increase this resolution to approximately 1-2 mm with minimal redesign, simply by reducing AHC spacing. 
Finally, the results validate the HFRD approach as well as the array concept. The HFRD method showed good performance and robustness even in cases where the output deviated from the method's simplifying assumptions. Its effectiveness could be improved by measuring a broader frequency spectrum and optimizing the level of frequency separation. The general method could be applied to more complex AHC sensing scenarios where one bilayer is used to measure multiple hair vibrations or more broadly to any vibration sensing application limited to one output channel. 


\section{Chapter 7}

\section{Summary and Conclusions}

\subsection{Research Summary}

The research in this document focused on the development of artificial hair cell (AHC) arrays for air flow sensing based on biomolecular transduction. It built upon the "membrane-based" AHC concept pioneered by Sarles and Leo [61, which demonstrated the ability of lipid bilayer membranes as vibration transducers. The work influenced the research presented here in two major ways: the use of droplet interface bilayers (DIBs) for ease of assembly and their physical encapsulation for portability. The encapsulation method, however, was modified considerably to allow for array fabrication. The modifications incorporated hydrogel supported DIBs, as developed by Holden et al. The technique simplified bilayer assembly and manipulation.

A primary goal of this research was to combine the concepts above to create a compact AHC unit, capable of modular assembly. In addition, we aimed to improve the response characteristics (i.e. sensitivity, signal to noise ratio, bilayer stability) over previous sensors and characterize them. By achieving these goals, we could demonstrate flow sensing with AHC arrays and develop a sensing strategy based on their properties. This would provide valuable insight into the transduction potentials and limits of bilayer membranes.

The final design featured slender, separated compartments to house a row of AHCs. Compartmentalized bilayers could be manipulated independently to create arrays. The freedom in the substrate design afforded by hydrogel supports played a key role in achieving modu- 
larity. A compact design was achieved without sacrificing control of individual bilayer sizes, one the key benefits of the regulated attachment method previously developed by Sarles [78]. The study of the sensor properties began in Chapter 4 with the temporal and spectral characterization of single AHC units. Laser vibrometry testing of the hair-hydrogel system alone revealed various bending modes of the hair within the bilayer's measurement range. Further investigation considered the AHC system as a whole and studied how the hair vibrations manifested in the membrane electrical output. This was done by striking both the hair and the microphone with a short pulse of air and estimating the hair cell's transfer function. A key observation from these tests was that the number of modes remained unchanged, although added mass from air and/or oil seemed to contribute to a slight downward shift in frequency.

In Chapter 5 the study focused on determining sensor sensitivity to two primary inputs: membrane voltage and airflow magnitude. The operating ranges of our study covered -100 to $100 \mathrm{mV}$ and 0 to $72 \mathrm{~m} / \mathrm{s}$. This work sought to determine analytical relationships between these variables and the current output. The extent of these relationships was explored both in the time domain, via the root mean squared amplitude (RMS), and in the frequency domain, via the power spectral density distribution (PSD). A convenient bridge between the domains is the amplitude spectral density, which could represent the amplitude contribution of specific modes. The conclusions from this portion of this study were used to build the set of assumptions for the array sensing process.

Array assembly and sensing was carried out in Chapter 6. Arrays of three units were built and tested against a laterally moving constant airflow. The array output measurement was limited to only one channel, which required a unique solution to resolve the outputs of individual hairs. To this end, we developed the hair frequency response decomposition method (HFRD) based on assumptions drawn from experimental observations in previous chapters. In HFRD the AHCs, each with a hair of different length, were multiplexed into one output channel and stored digitally. A least squares fit of AHC reference signals to the output data determined which AHCs were excited. Then, a linear interpolation calculated the excitation magnitude based on the previously determined sensitivity properties. The end result was a mapping of frequency data to the spacial location of the flow stimuli. To the author's knowledge, this is the first demonstration of stimulus localization using arrays of membrane based AHCs. 


\subsection{Conclusions}

An incremental research approach was used to take existing concepts in bilayer based transduction, refine them into a compact artificial hair cell unit design, characterize it and implement an array sensing method. The conclusions drawn from this research are listed below:

\subsubsection{AHC Design and Characterization}

- Two sources of undesired electrical noise were identified in AHC sensors: power line and electrode noise. Applied membrane voltage was shown to amplify the bilayer signal without affecting these sources, providing a simple an effective method to improve AHC signal to noise ratio. Control tests on the bilayer output showed signal to noise ratios 16 and above, indicating good signal clarity. Such clarity made good characterization and frequency decomposition results possible.

- The mechanical properties of the AHC system, namely hydrogel stiffness, hair stiffness and length, are within such a range that airflow induces free bending vibrations. Characterization of the AHC via base excitation and air pulses in the 5-2,000 $\mathrm{Hz}$ spectrum show that membrane current oscillations match the vibrational modes of the hair. As one would expect, the current's frequency response is more reflective of vibrations near the base of the hair than the hair as a whole.

- Multiple instances of divergence in the second bending mode highlight the complexity of the hydrogel base boundary condition. This behavior is attributed to asymmetric stiffness resulting from its non-homogeneous material properties. A Rayleigh-Ritz tapered beam approximation yields estimates of $k=19 \mathrm{~N} / \mathrm{m}$ and $\tau=0.0011 \mathrm{Nm} / \mathrm{rad}$ for the equivalent linear and torsional stiffness of the gel in the primary sensing direction.

- Peak AHC current responses in the nanoamp range have been consistently recorded, an order of magnitude increase over current sensors of its kind. A significant factor in this improvement is believed to be the stiffness of the glass fiber hairs, which is much higher than previously used polymer hairs. Just like in biological hair cells, greater hair stiffness helps power transfer from air to hair, increasing sensitivity. Most flowsensing biological hairs, in fact, undergo rigid deflection. Subsequent designs should also seek to meet this condition for even higher output amplitudes. The lack of bending 
modes would result in only one or two frequencies (rigid modes) and could make HFRD implementation more straightforward. However, it is unclear if a rigid hair would only deflect and not vibrate at all, invalidating and HFRD analysis.

\subsubsection{AHC Sensitivity Properties}

- Electrowetting sensitivity $\left(\alpha=21.5 \mathrm{~V}^{-2}\right)$ matches closely with other studies but reaches a plateau $\left(\alpha \approx 4 \mathrm{~V}^{-2}\right)$ above approximately $40 \mathrm{mV}$. This saturation obscures the cubic dependence of current on voltage and leads to a strong linear relationship. We conclude that the hydrogel supports place constraints on lipid droplet movements which counteract bilayer growth due to electrowetting. The hydrogels in essence, help to maintain capacitance constant. This is an advantageous effect because it simplifies the voltage sensitivity relationship.

- Not only does applied membrane voltage increase overall sensitivity in a linear fashion, it does so at the spectral level as well. The modal magnitudes reflected by the membrane current are scaled uniformly. Thus, voltage acts simply as a linear gain for bilayer oscillatory output and has no noticeable effects on the dynamics.

- The total current output magnitude (amplitude spectral density) increases linearly with airflow velocity. Similar to the voltage case, individual modal ASDs also scale uniformly within the measurement range $(0-72 \mathrm{~m} / \mathrm{s})$. Slight deviations from the rule are observed at high velocities $(>50 \mathrm{~m} / \mathrm{s})$. The total ASD, however, always remains highly linear. We conclude that the total power transfer by the airflow to the hair is proportional to its velocity but may redistribute to higher order modes as the velocity increases. This behavior limits the effectiveness of the HFRD method.

- Considering bilayer tranduction capabilities, we find that the AHC is able to withstand airflow as fast as $72 \mathrm{~m} / \mathrm{s}$. It can clearly transduce vibrations in the range of 200-2,000 Hz. The upper limit is simply the Nyquist frequency our measurements, it is possible that transduction could occur much higher (bilayers display capacitive behavior as high as $10,000 \mathrm{~Hz}$ ). The lower limit could represent a loss of effective mechanical coupling between the hair and the hydrogel, where vibrations are no longer felt by the bilayer. 


\subsubsection{Array Implementation}

- Hair frequency response decomposition is a robust method for separating spectral information into component signals. It showed accurate localization of a flow source despite overlapping frequency responses. The method could be expanded to analyze signals of more complex arrays or networks.

- The bilayer, as an organic structure, is more variable than traditional mechanoelectrical components. Changes in bilayer size throughout array testing caused fluctuations in sensitivity that made it difficult to estimate airflow velocity with certainty. Fortunately, sensitivity is just an electrical property of the AHC and changes in membrane size had little effect on the modal behavior of the output. Nevertheless, addressing this issue is difficult because it is a result of one of the most beneficial properties of bilayer, selfassembly. Vibrations can lead a membrane to rearrange from its initial configuration into a more favorable one. This is challenging to counteract without very precise constraints on bilayer movement.

- This work has shown it is possible to make modular membrane based AHCs, while still improving their output characteristics, by incorporating hydrogels. AHC modularity made arrays possible but only in small numbers (two or three). More sophisticated and precise techniques, instead of hand fabrication, are necessary to increase this number.

\subsection{Contributions}

The following key contributions from this research advance upon previously demonstrated capabilities of lipid bilayers in a sensory context:

- Development of an artificial hair cell platform based on fully hydrogel supported lipid bilayer membranes. This design had two distinct advantages. First, bilayers could be manipulated and oriented freely via the hydrogels, without the need for a contacting substrate. The flexibility in layout facilitated the creation of compact, modular units. Second, hydrogel size was adjusted to accommodate stiffer hairs, which led to improved signal to noise ratios. Nanoamp range output currents were consistently observed, higher that any membrane based sensor to date. Overall, the sensor is an excellent 
platform for studying bilayer membrane response to many stimuli (water flow, touch, etc.).

- Spectral characterization methods of an AHC response in airflow. These methods were able to calculate a clear input-output relationship for a delicate and complex dynamic system, which was difficult to interrogate by traditional modal analysis techniques. The methodology was used to understand bilayer behavior, but could be easily expanded to the study of small hair cells in general.

- Array implementation of independently controlled bilayer-based artificial hair cells. This includes the development of a novel method of frequency to location mapping whose strategy involved using hairs of different lengths. This is the first demonstration of using array of this type of sensor for stimulus localization. 


\section{Bibliography}

[1] Nicolas Franceschini, Jean-Marc Pichon, Christian Blanes, and JM Brady. From insect vision to robot vision [and discussion]. Philosophical Transactions of The Royal Society Of London. Series B: Biological Sciences, 337(1281):283-294, 1992.

[2] Nicolas Franceschini. Sensors and sensing in biology and engineering. Springer, 2003.

[3] Ben M Clopton and Herbert F Voigt. The peripheral auditory system. 2006.

[4] GM Sessler. Acoustic sensors. Sensors and Actuators A: Physical, 26(1):323-330, 1991.

[5] ME Del Valle, Teresa Cobo, JL Cobo, and JA Vega. Mechanosensory neurons, cutaneous mechanoreceptors, and putative mechanoproteins. Microscopy research and technique, 75(8):1033-1043, 2012.

[6] Friedrich G Barth, Joseph AC Humphrey, and Timothy W Secomb. Sensors and sensing in biology and engineering. Springer, 2003.

[7] H Ti Tien and Angelica Ottova-Leitmannova. Planar Lipid Bilayers (BLM's) and Their Applications.

[8] Sanjay P. Sane and Matthew J. McHenry. The biomechanics of sensory organs. In Sensory Biomechanics, pages i8-i23. Society for Integrative and Comparative Biology, Oxford University Press, 2009.

[9] Albert, Friedrich, Dechant, and et al. Arthropod touch reception: spider hair sensilla as rapid touch detectors. Journal of Comparative Physiology A: Neuroethology, Sensory, Neural, and Behavioral Physiology, 187(4):303-312, 2001.

[10] FG Barth. How to catch the wind: spider hairs specialized for sensing the movement of air. Naturwissenschaften, 87(2):51-58, 2000.

[11] J. Montgomery, S. Coombs, and M. Halstead. Biology of the mechanosensory lateral line in fishes. Reviews in Fish Biology and Fisheries, 5:399-416, 1995.

[12] A. J. Hudspeth. How the ear's works work. Nature, 341:397-404, 1989. 
[13] James O Pickles and David P Corey. Mechanoelectrical transduction by hair cells. Trends in neurosciences, 15(7):254-259, 1992.

[14] T Teyke. Collision with and avoidance of obstacles by blind cave fishanoptichthys jordani (characidae). Journal of Comparative Physiology A, 157(6):837-843, 1985.

[15] Michael E McConney, Nannan Chen, David Lu, Huan A Hu, Sheryl Coombs, Chang Liu, and Vladimir V Tsukruk. Biologically inspired design of hydrogel-capped hair sensors for enhanced underwater flow detection. Soft Matter, 5(2):292-295, 2009.

[16] Sheryl Coombs and Sietse Van Netten. The hydrodynamics and structural mechanics of the lateral line system. Fish physiology, 23:103, 2006.

[17] Friedrich G Barth. Spider mechanoreceptors. Current opinion in neurobiology, 14(4):415-422, 2004.

[18] Friedrich G Barth and Hans-Erich Dechant. Sensors and sensing in biology and engineering. Springer, 2003.

[19] Joseph AC Humphrey, Friedrich G Barth, Michael Reed, and Aaron Spak. Sensors and sensing in biology and engineering. Springer, 2003.

[20] Junliang Tao and Xiong Bill Yu. Hair flow sensors: from bio-inspiration to biomimickinga review. Smart Materials and Structures, 21(11):113001, 2012.

[21] Joseph AC Humphrey, Raghuram Devarakonda, Immaculada Iglesias, and Friedrich G Barth. Dynamics of arthropod filiform hairs. i. mathematical modelling of the hair and air motions. Philosophical Transactions of the Royal Society of London. Series B: Biological Sciences, 340(1294):423-444, 1993.

[22] Luis Robles and Mario A. Ruggero. Mechanics of the mammalian cochlea. Physiological Reviews, 81(3):1305-1352, 2001.

[23] Jont B Allen. Cochlear micromechanicsa physical model of transduction. The Journal of the Acoustical Society of America, 68(6):1660-1670, 1980.

[24] J. J. Zwislocki. Five decades of research on cochlear mechanics. The Journal of the Acoustical Society of America, 67(5):1679-1685, 1980.

[25] G v Békésy. Description of some mechanical properties of the organ of corti. The Journal of the Acoustical Society of America, 25(4):770-785, 1953.

[26] Donald D Greenwood. Critical bandwidth and the frequency coordinates of the basilar membrane. The Journal of the Acoustical Society of America, 33(10):1344-1356, 1961.

[27] Sven Dijkgraaf. The functioning and significance of the lateral-line organs. Biological Reviews, 38(1):51-105, 1963. 
[28] Brian L Partridge and Tony J Pitcher. The sensory basis of fish schools: relative roles of lateral line and vision. Journal of Comparative Physiology, 135(4):315-325, 1980.

[29] Denise Hoekstra and John Janssen. Non-visual feeding behavior of the mottled sculpin, cottus bairdi, in lake michigan. Environmental biology of fishes, 12(2):111-117, 1985.

[30] M Satou, H-A Takeuchi, J Nishii, M Tanabe, S Kitamura, N Okumoto, and M Iwata. Behavioral and electrophysiological evidences that the lateral line is involved in the inter-sexual vibrational communication of the himé salmon (landlocked red salmon, oncorhynchus nerka). Journal of Comparative Physiology A, 174(5):539-549, 1994.

[31] AM Sutterlin and Susan Waddy. Possible role of the posterior lateral line in obstacle entrainment by brook trout (salvelinus fontinalis). Journal of the Fisheries Board of Canada, 32(12):2441-2446, 1975.

[32] John C Montgomery, Cindy F Baker, and Alexander G Carton. The lateral line can mediate rheotaxis in fish. Nature, 389(6654):960-963, 1997.

[33] Joachim Mogdans, Jacob Engelmann, Wolf Hanke, and Sophia Krother. Sensors and sensing in biology and engineering. Springer, 2003.

[34] John Montgomery, Sheryl Coombs, and Matthew Halstead. Biology of the mechanosensory lateral line in fishes. Reviews in Fish Biology and Fisheries, 5(4):399-416, 1995.

[35] Jacob Engelmann, Wolf Hanke, Joachim Mogdans, and Horst Bleckmann. Neurobiology: Hydrodynamic stimuli and the fish lateral line. Nature, 408(6808):51-52, 2000.

[36] Tibor Hianik and Victor Ivanovich Passechnik. Bilayer lipid membranes. Structure and mechanical properties. Springer, 1995.

[37] J. Katsaras and T. Gutberlet. Lipid Bilayers. Springer-Verlag Berlin Heidelberg, 2000.

[38] Fritz A Henn and TE Thompson. Synthetic lipid bilayer membranes. Annual review of biochemistry, 38(1):241-262, 1969.

[39] Edward T Castellana and Paul S Cremer. Solid supported lipid bilayers: From biophysical studies to sensor design. Surface Science Reports, 61(10):429-444, 2006.

[40] Paul Mueller, DONALD O RUDIN, H Ti Tien, and WILLIAM C WESCOTT. Reconstitution of excitable cell membrane structure in vitro. Circulation, 26(5):1167-1171, 1962.

[41] Dimitrios P Nikolelis and Christina G Siontorou. Bilayer lipid membranes for flow injection monitoring of acetylcholine, urea, and penicillin. Analytical chemistry, 67(5):936944, 1995. 
[42] M Montal and P Mueller. Formation of bimolecular membranes from lipid monolayers and a study of their electrical properties. Proceedings of the National Academy of Sciences, 69(12):3561-3566, 1972.

[43] R Naumann, T Baumgart, P Gräber, A Jonczyk, A Offenhäusser, and W Knoll. Proton transport through a peptide-tethered bilayer lipid membrane by the $h_{i} \sup i+i / \sup i-$ atp synthase from chloroplasts measured by impedance spectroscopy. Biosensors and Bioelectronics, 17(1):25-34, 2002.

[44] A Sonnleitner, GJ Schütz, and Th Schmidt. Free brownian motion of individual lipid molecules in biomembranes. Biophysical journal, 77(5):2638-2642, 1999.

[45] James R Thompson, Andrew J Heron, Yusdi Santoso, and Mark I Wallace. Enhanced stability and fluidity in droplet on hydrogel bilayers for measuring membrane protein diffusion. Nano letters, 7(12):3875-3878, 2007.

[46] Stephen A Sarles. Physical encapsulation of Interface Bilayers. PhD dissertation, Virginia Polytechnic Institute, 2010.

[47] WM Reichert, CJ Bruckner, and J Joseph. Langmuir-blodgett films and black lipid membranes in biospecific surface-selective sensors. Thin Solid Films, 152(1):345-376, 1987.

[48] Kei Funakoshi, Hiroaki Suzuki, and Shoji Takeuchi. Lipid bilayer formation by contacting monolayers in a microfluidic device for membrane protein analysis. Analytical chemistry, 78(24):8169-8174, 2006.

[49] Hagan Bayley, Brid Cronin, Andrew Heron, Matthew A Holden, William L Hwang, Ruhma Syeda, James Thompson, and Mark Wallace. Droplet interface bilayers. Molecular BioSystems, 4(12):1191-1208, 2008.

[50] Matthew A Holden, David Needham, and Hagan Bayley. Functional bionetworks from nanoliter water droplets. Journal of the American Chemical Society, 129(27):8650$8655,2007$.

[51] K. Tanuj Sapra and Hagan Bayley. Lipid-coated hydrogel shapes a components of electrical circuits and mechanical devices. Nature, 2:1-9, 2012.

[52] Stephen A. Sarles, L. Justin Stiltner, Chris B. Williams, and Donald J. Leo. Bilayer formation netween lipid-encased hydrogels contained in solid substrates. Applied Materials and Interfaces, 2(12):36543663, 2011.

[53] Rodrigo Sarlo and Donald Leo. Directional sensitivity analysis of a hydrogel-supported artificial hair cell. In ASME 2013 Conference on Smart Materials, Adaptive Structures and Intelligent Systems, pages V002T06A016-V002T06A016. American Society of Mechanical Engineers, 2013. 
[54] Alexander G Petrov. Planar Lipid Bilayers (BLM's) and Their Applications.

[55] Alfred L Ochs and Robert M Burton. Electrical response to vibration of a lipid bilayer membrane. Biophysical journal, 14(6):473-489, 1974.

[56] Srikoundinya Punnamaraju and Andrew J Steckl. Voltage control of droplet interface bilayer lipid membrane dimensions. Langmuir, 27(2):618-626, 2010.

[57] AG Petrov and VS Sokolov. Curvature-electric effect in black lipid membranes. European Biophysics Journal, 13(3):139-155, 1986.

[58] Boris Martinac. Mechanosensitive ion channels. Channels, 6(4):211-213, 2012.

[59] VS Markin and B Martinac. Mechanosensitive ion channels as reporters of bilayer expansion. a theoretical model. Biophysical journal, 60(5):1120-1127, 1991.

[60] Donald J Leo. Engineering analysis of smart material systems. John Wiley \& Sons, Inc, 2007.

[61] Stephen A Sarles, John DW Madden, and Donald J Leo. Hair cell inspired mechanotransduction with a gel-supported, artificial lipid membrane. Soft Matter, 7(10):46444653, 2011.

[62] Chang Liu. Micromachined biomimetic artificial haircell sensors. Bioinspiration and Biomimetics, 2:S162-S169, 2007.

[63] G. J. Krijnen and et al. Mems based hair flow-sensors as model systems for acoustic percetion studies. Nanotechnology, 17:S84-S89, 2006.

[64] Jack Chen, Jonathan Engel, Nannan Chen, Saunvit Pandya, Sheryl Coombs, and Chang Liu. Artificial lateral line and hydrodynamic object tracking. In Micro Electro Mechanical Systems, 2006. MEMS 2006 Istanbul. 19th IEEE International Conference on, pages 694-697. IEEE, 2006.

[65] Patrick D McGary, Liwen Tan, Jia Zou, Bethanie JH Stadler, Patrick R Downey, and Alison B Flatau. Magnetic nanowires for acoustic sensors. Journal of Applied Physics, 99(8):08B310, 2006.

[66] Xiong Yu, Junliang Tao, and Jim Berilla. A bio-inspired flow sensor. In SPIE Smart Structures and Materials + Nondestructive Evaluation and Health Monitoring, pages 764618-764618. International Society for Optics and Photonics, 2010.

[67] Ch Brücker, J Spatz, and W Schröder. Feasability study of wall shear stress imaging using microstructured surfaces with flexible micropillars. Experiments in fluids, 39(2):464-474, 2005. 
[68] JM Engel, J Chen, D Bullen, and C Liu. Polyurethane rubber as a mems material: characterization and demonstration of an all-polymer two-axis artificial hair cell flow sensor. In Micro Electro Mechanical Systems, 2005. MEMS 2005. 18th IEEE International Conference on, pages 279-282. IEEE, 2005.

[69] Jonathan M Engel, Jack Chen, Chang Liu, and David Bullen. Polyurethane rubber all-polymer artificial hair cell sensor. Microelectromechanical Systems, Journal of, 15(4):729-736, 2006.

[70] Nannan Chen, Craig Tucker, Jonathan M Engel, Yingchen Yang, Saunvit Pandya, and Chang Liu. Design and characterization of artificial haircell sensor for flow sensing with ultrahigh velocity and angular sensitivity. Microelectromechanical Systems, Journal of, 16(5):999-1014, 2007.

[71] Sergiy Pelechanko and et al. Hydrogel-encapsulated microfabricated haircells mimicking fish cupula neuromast. Advanced Materials, 19:2903-2909, 2007.

[72] M Dijkstra, JJ Van Baar, RJ Wiegerink, TSJ Lammerink, JH De Boer, and GJM Krijnen. Artificial sensory hairs based on the flow sensitive receptor hairs of crickets. Journal of micromechanics and microengineering, 15(7):S132, 2005.

[73] RK Jaganatharaja, CM Bruinink, BM Hagedoorn, ML Kolster, TSJ Lammerink, RJ Wiegerink, and GJM Krijnen. Highly-sensitive, biomimetic hair sensor arrays for sensing low-frequency air flows. In Solid-State Sensors, Actuators and Microsystems Conference, 2009. TRANSDUCERS 2009. International, pages 1541-1544. IEEE, 2009.

[74] Remco J Wiegerink, Arjan Floris, Ram K Jaganatharaja, Nima Izadi, Theo SJ Lammerink, and Gijs JM Krijnen. Biomimetic flow-sensor arrays based on the filiform hairs on the cerci of crickets. In Sensors, 2007 IEEE, pages 1073-1076. IEEE, 2007.

[75] Yingchen Yang, Jack Chen, Jonathan Engel, Saunvit Pandya, Nannan Chen, Craig Tucker, Sheryl Coombs, Douglas L. Jones, and Chang Liu. Distant touch hydrodynamic imaging with an artificial lateral line. Proceedings of the National Academy of Sciences, 103(50):18891-18895, 2006.

[76] Brice Bathellier, Friedrich G Barth, Jörg T Albert, and Joseph AC Humphrey. Viscosity-mediated motion coupling between pairs of trichobothria on the leg of the spider cupiennius salei. Journal of comparative physiology A, 191(8):733-746, 2005.

[77] Stephen A Sarles and Donald J Leo. Membrane-based biomolecular smart materials. Smart materials and structures, 20(9):094018, 2011.

[78] Stephen A Sarles and Donald J Leo. Regulated attachment method for reconstituting lipid bilayers of prescribed size within flexible substrates. Analytical chemistry, 82(3):959-966, 2010. 
[79] Preston A Pinto, Kevin Garrison, Donald J Leo, and Stephen A Sarles. A bio-inspired aquatic flow sensor using an artificial cell membrane. SPIE Smart Structures and Materials+ Nondestructive Evaluation and Health Monitoring, pages 833907-833907, 2012.

[80] Kevin Lee Garrison. Design, fabrication, and validation of membrane-based sensors. 2012.

[81] Nima Tamaddoni and Andy Sarles. Fabrication and characterization of a membrane based hair cell sensor that features soft hydrogel materials. In ASME 2012 Conference on Smart Materials, Adaptive Structures and Intelligent Systems, pages 565-571. American Society of Mechanical Engineers, 2012.

[82] Nima Tamaddoni and Andy Sarles. Characterizing the sources of current generated by a membrane-based hair cell sensor. In ASME 2013 Conference on Smart Materials, Adaptive Structures and Intelligent Systems, pages V002T06A015-V002T06A015. American Society of Mechanical Engineers, 2013.

[83] Jeffrey P Travis, Myles D Dunlap, Donald J Leo, and J Wallace Grant. Dynamic characterization of biomimetic artificial hair cells. In ASME 2013 Conference on Smart Materials, Adaptive Structures and Intelligent Systems, pages V002T06A011V002T06A011. American Society of Mechanical Engineers, 2013.

[84] Stephen A Sarles, Kevin L Garrison, Taylor T Young, and Donald J Leo. Formation and encapsulation of biomolecular arrays for developing arrays of membrane-based artificial hair cell sensors. In ASME 2011 Conference on Smart Materials, Adaptive Structures and Intelligent Systems, pages 663-671. American Society of Mechanical Engineers, 2011.

[85] Nima Tamaddoni and Stephen A Sarles. Mechanotransduction of multi-hair droplet arrays. In ASME 2014 Conference on Smart Materials, Adaptive Structures and Intelligent Systems, pages V002T06A008-V002T06A008. American Society of Mechanical Engineers, 2014.

[86] William L Hwang, Min Chen, Brid Cronin, Matthew A Holden, and Hagan Bayley. Asymmetric droplet interface bilayers. Journal of the American Chemical Society, 130(18):5878-5879, 2008.

[87] Myles D. Dunlap. Experimental Measurement of the Utricles Dynamic Response and the Mechanoelectrical Characterization of a Micron-Sized Droplet Interface Bilayer. $\mathrm{PhD}$ thesis, Virginia Polytechnic Institute and State University, 2013.

[88] Brian J Polk, Anna Stelzenmuller, Geraldine Mijares, William MacCrehan, and Michael Gaitan. Ag/agcl microelectrodes with improved stability for microfluidics. Sensors and Actuators B: Chemical, 114(1):239-247, 2006. 
[89] Tatsuya Kitade, Keisuke Kitamura, Shigehiko Takegami, Yoko Miyata, Miwa Nagatomo, Tomomi Sakaguchi, and Michiko Furukawa. Needle-type ultra micro silver/silver chloride reference electrode for use in micro-electrochemistry. Analytical sciences, 21(8):907-912, 2005.

[90] James V Halliwell, Tim D Plant, Jon Robbins, and Nick B Standen. Voltage clamp techniques. In Microelectrode Techniques. The Plymouth Workshop Handbook, pages 17-35, 1994.

[91] Stephen A Sarles and Donald J Leo. Tailored current-voltage relationships of dropletinterface bilayers using biomolecules and external feedback control. Journal of intelligent material systems and structures, 2009.

[92] Leonard Meirovitch. Fundamentals of vibrations. Waveland Press, 2010.

[93] Winfried Römer and Claudia Steinem. Impedance analysis and single-channel recordings on nano-black lipid membranes based on porous alumina. Biophysical journal, 86(2):955-965, 2004.

[94] O Alvarez and R Latorre. Voltage-dependent capacitance in lipid bilayers made from monolayers. Biophysical journal, 21(1):1, 1978.

[95] Stephen H White and TE Thompson. Capacitance, area, and thickness variations in thin lipid films. Biochimica et Biophysica Acta (BBA)-Biomembranes, 323(1):7-22, 1973.

[96] Ezra Jamploe, Noel Spurgeon, Trevor Avant, and Kevin Farinholt. Characterization of Bio-Inspired Hair Cell Sensors, volume 6, chapter 13, pages 137-152. The Society for Experimental Mechanics, Inc., 2012.

[97] SH White and W Chang. Voltage dependence of the capacitance and area of black lipid membranes. Biophysical journal, 36(2):449, 1981.

[98] Leonard Meirovitch. Fundamentals of vibrations. Waveland Press, 2010.

[99] Paul Scherz, Simon Monk, Michael Margolis, and Chris Fitzer. Practical Electronics for Inventors. McGraw-Hill, 2013.

[100] TF Coleman and Y Li. On the convergence of reflective newton methods for large-scale nonlinear minimization subject to bounds. Mathematical Programming, 67(2):189-224, 1994.

[101] TF Coleman and Y Li. An interior, trust region approach for nonlinear minimization subject to bounds. SIAM Journal on Optimization, 6:418-445, 1994.

[102] Nallamuthu Rajaratnam. Turbulent Jets. Elsevier, 1976. 


\section{Appendix A}

\section{Substrate Dimensions}

A fully dimensioned diagram of the artificial hair cell array substrate is included for reference below: 


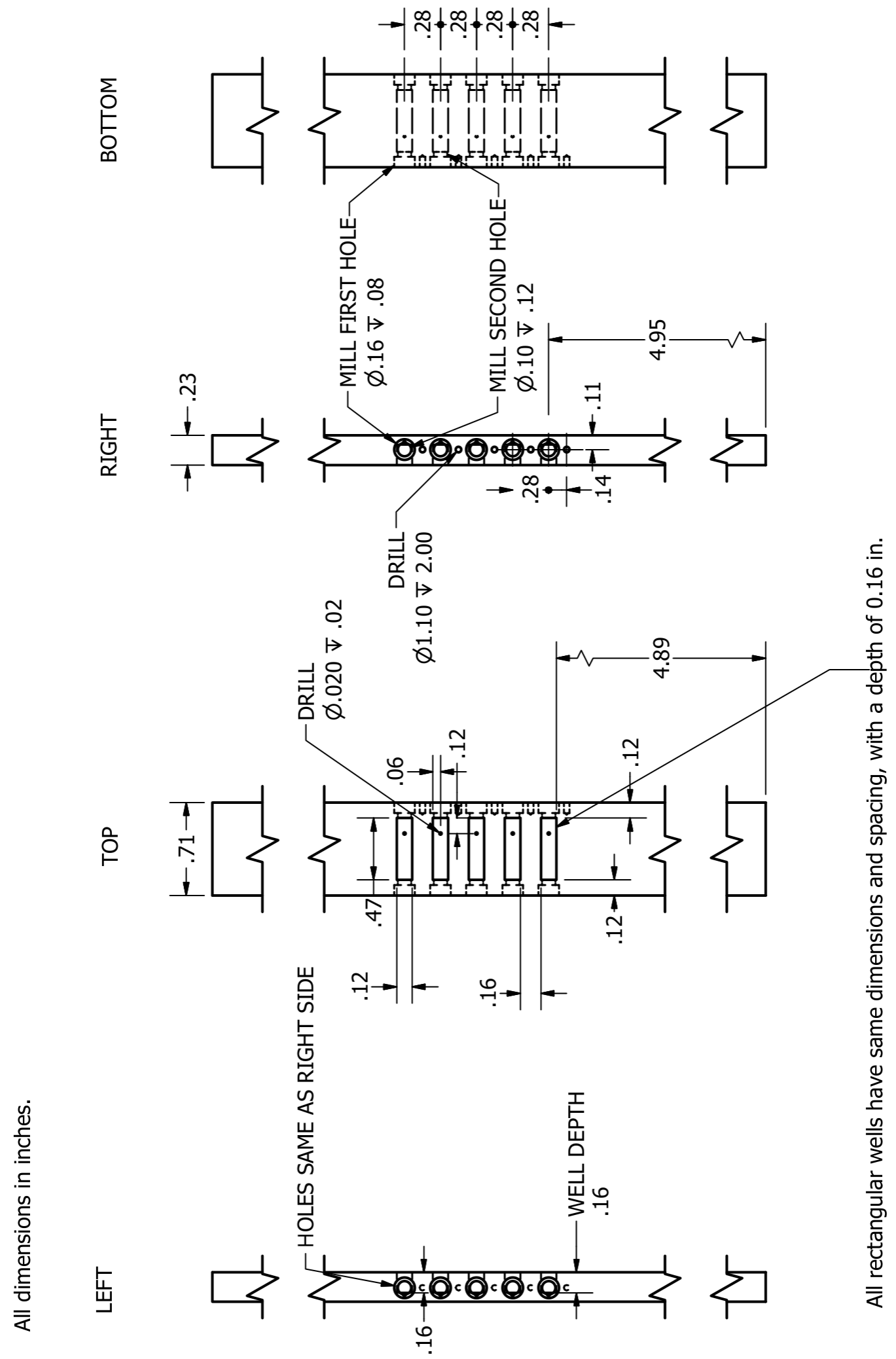




\section{Appendix B}

\section{Rayleigh-Ritz Calculation Algorithm}

The MATLAB implementation of the Rayleigh-Ritz approximation method described in Chapter 3 is included below:

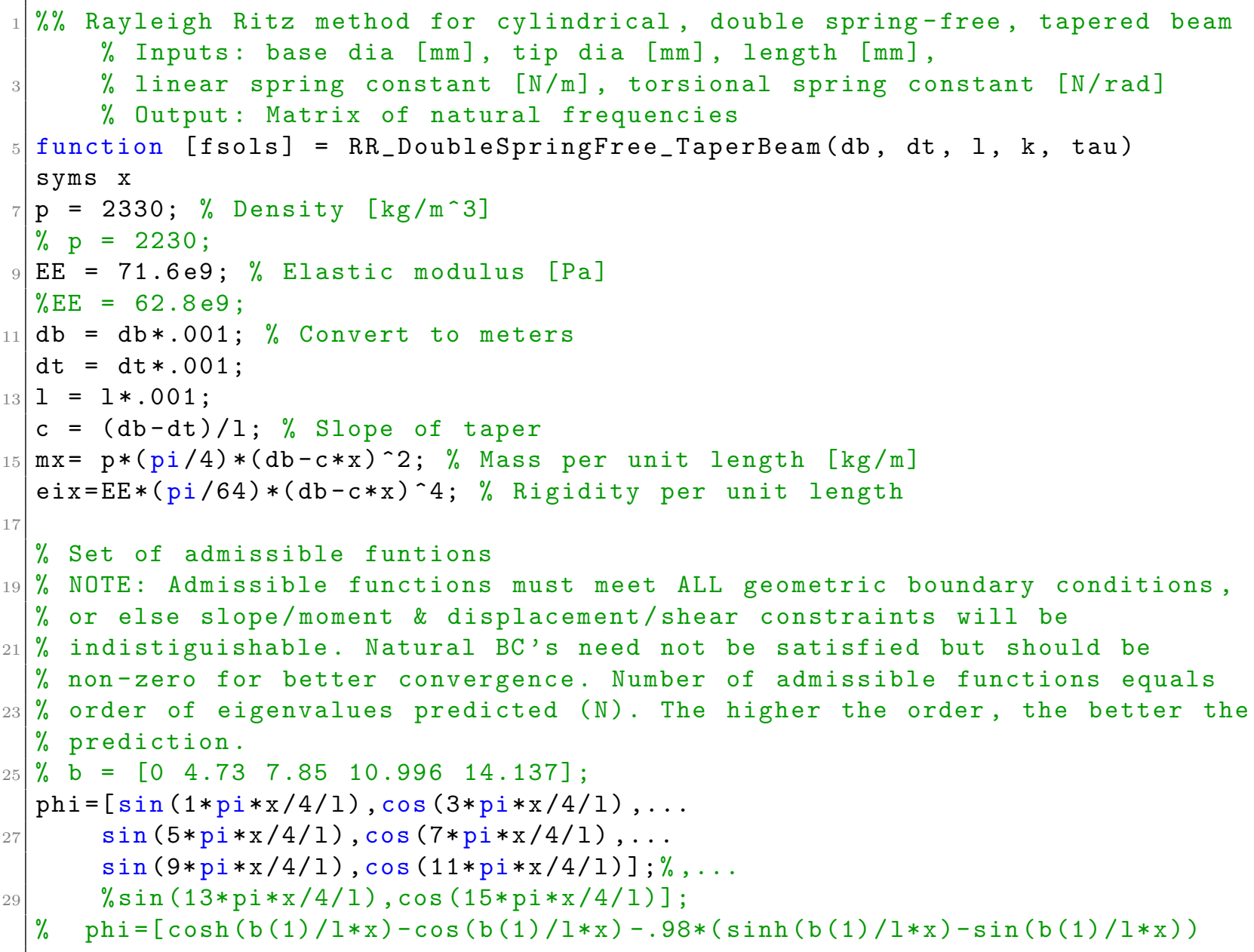




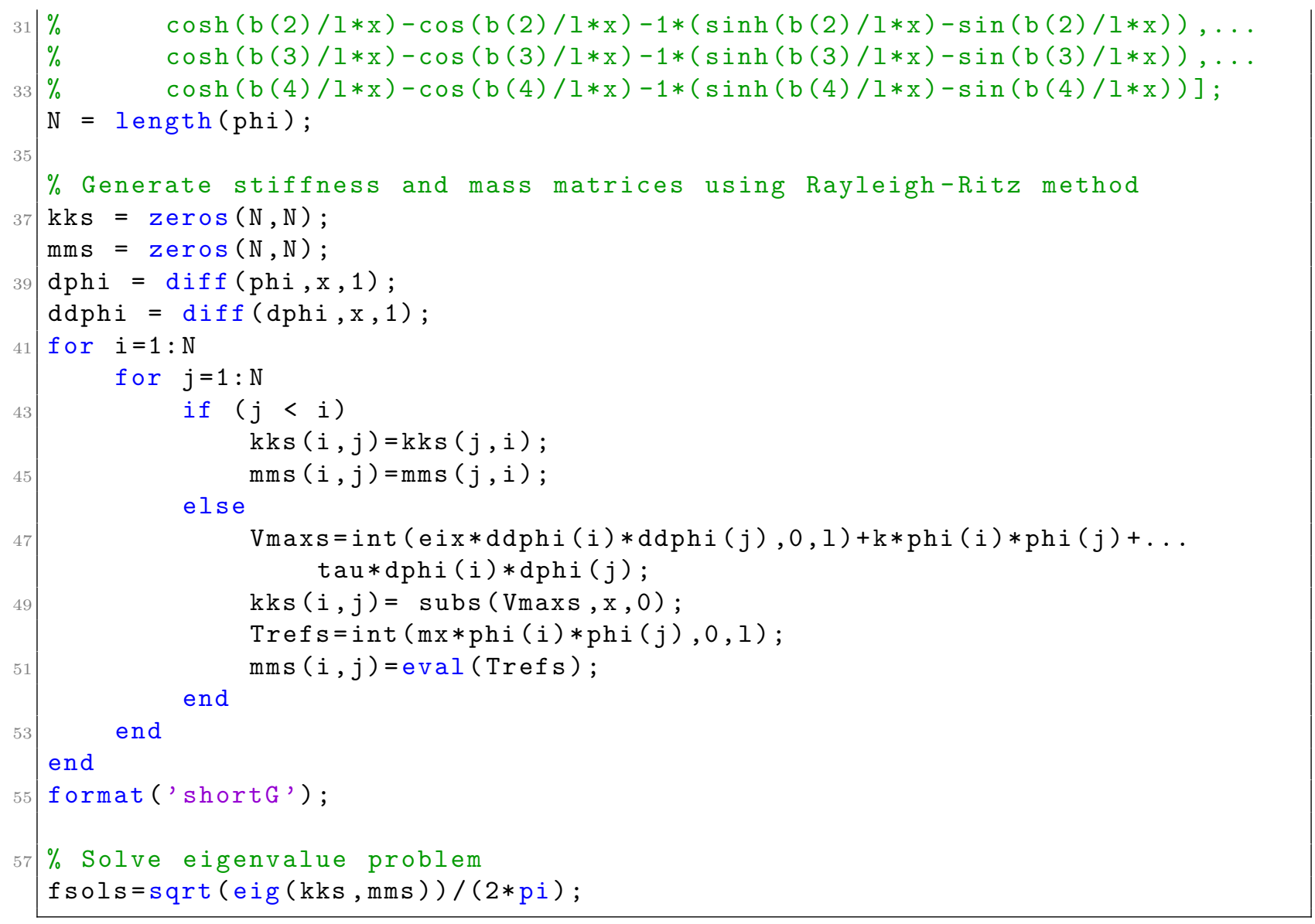




\section{Appendix C}

\section{Hair Frequency Response Decomposition Top-Level Algorithm}

The top level MATLAB code to implement the hair frequency decomposition algorithm described in Chapter 6 is included below:

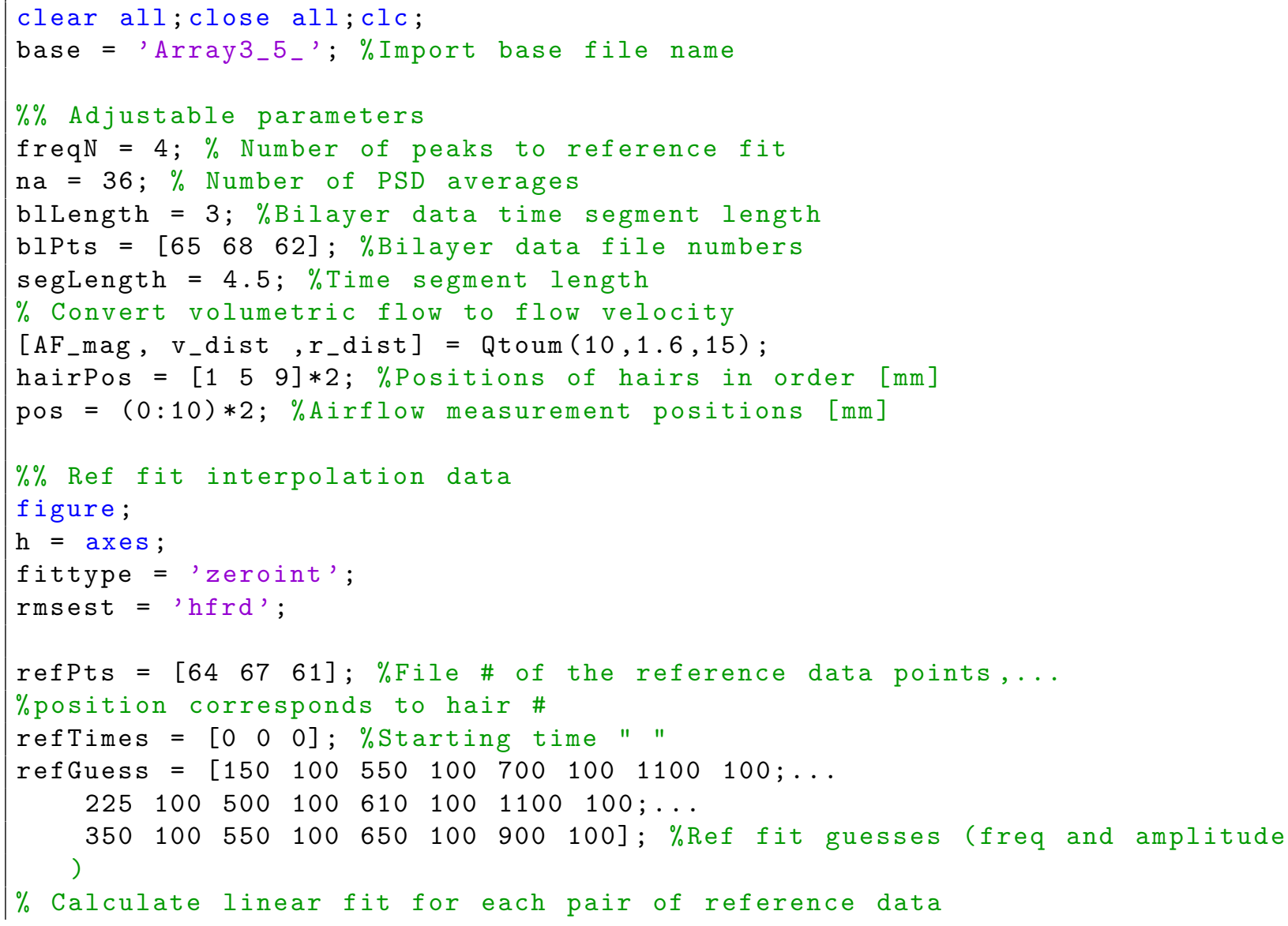


Rodrigo Sarlo App C: Hair Frequency Response Decomposition Top-Level Algorithm 103

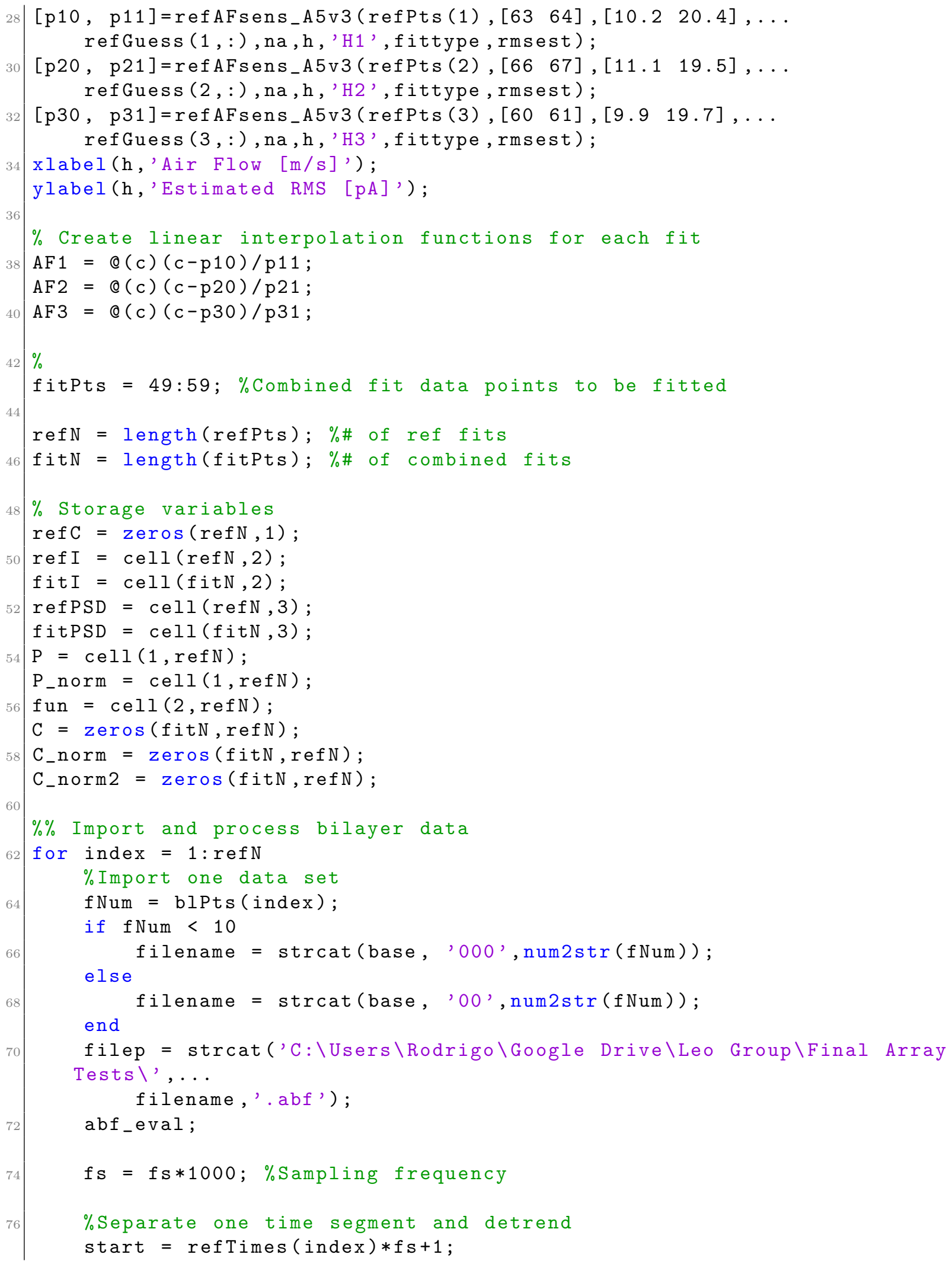




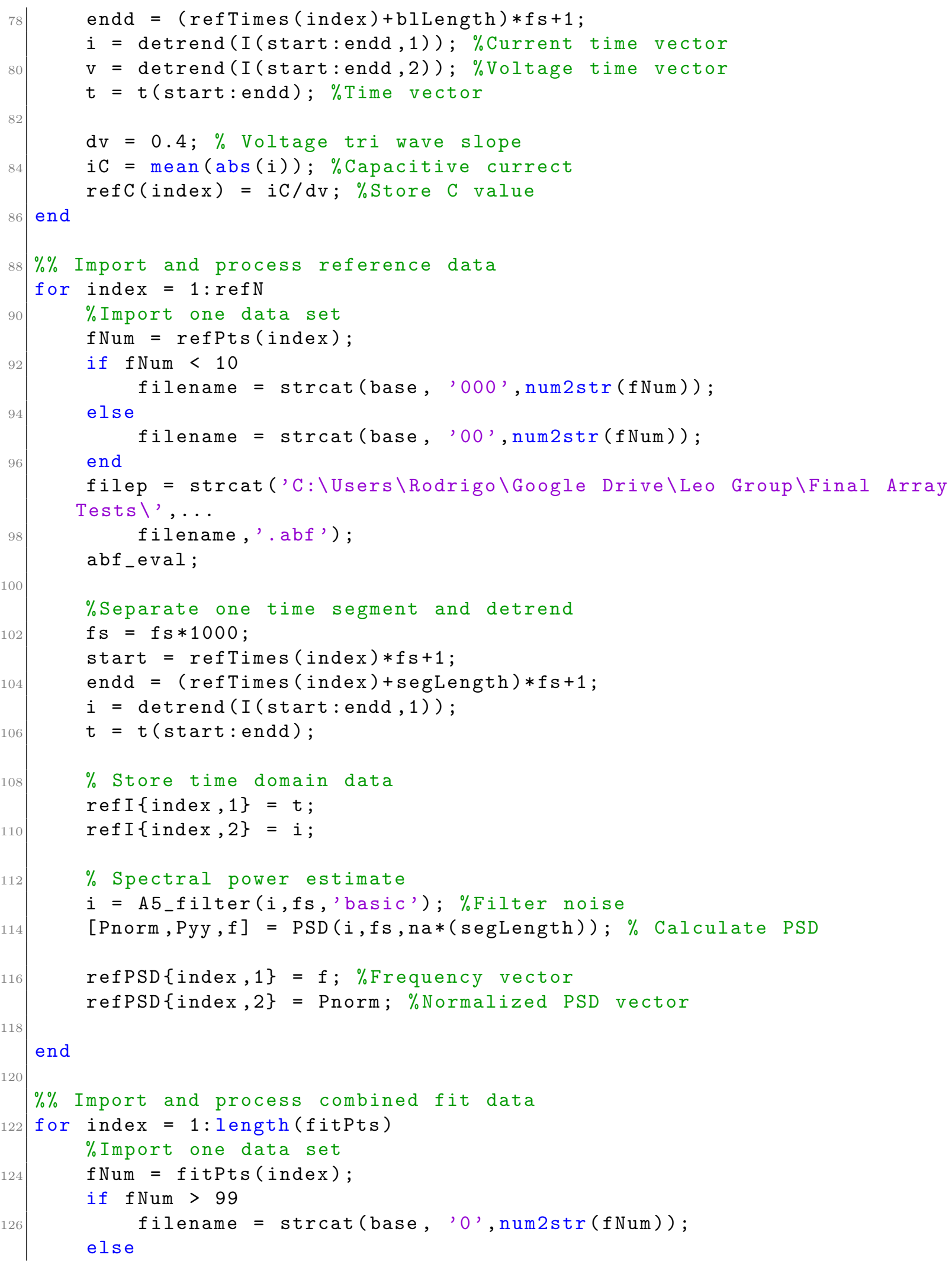




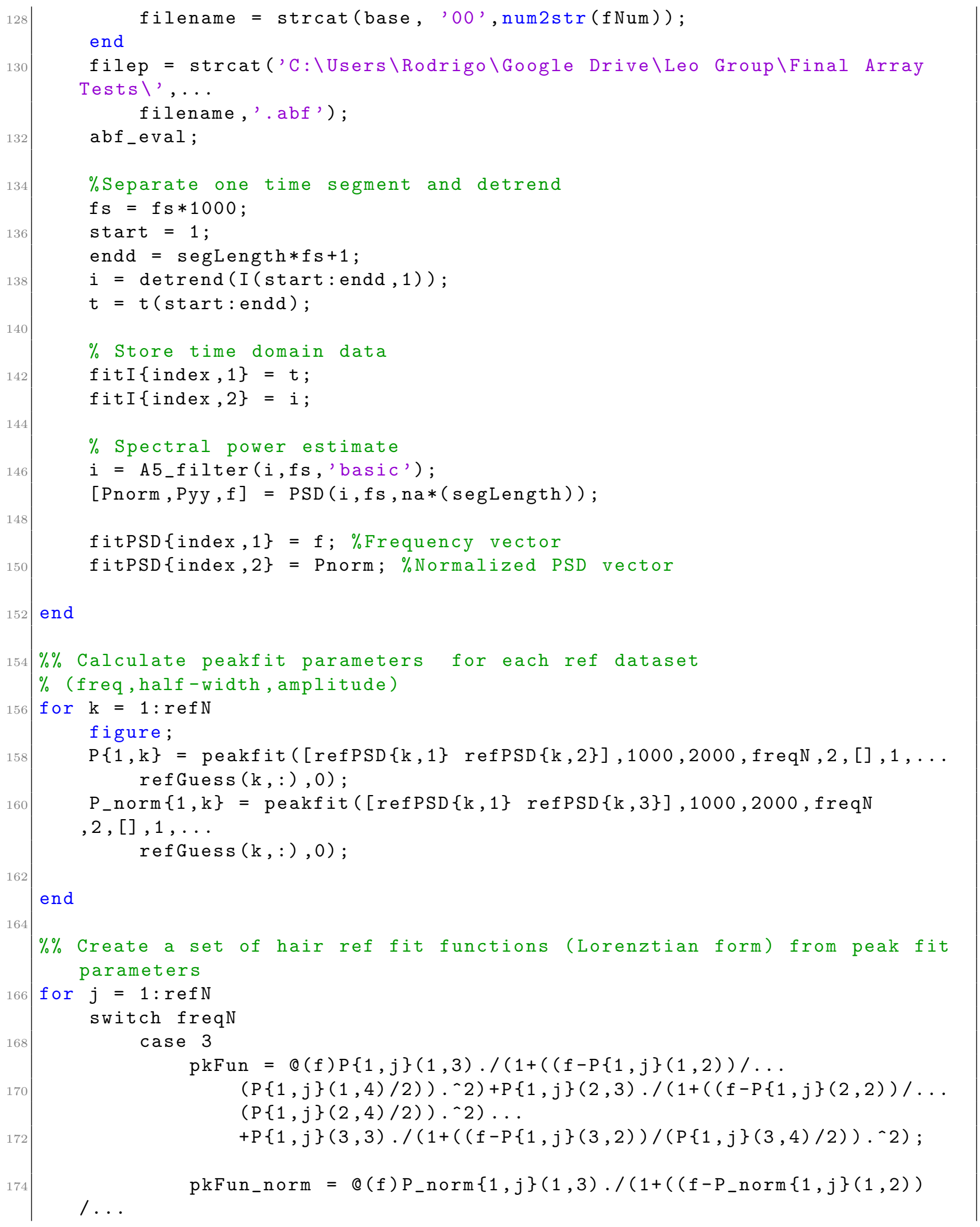


Rodrigo Sarlo App C: Hair Frequency Response Decomposition Top-Level Algorithm 106

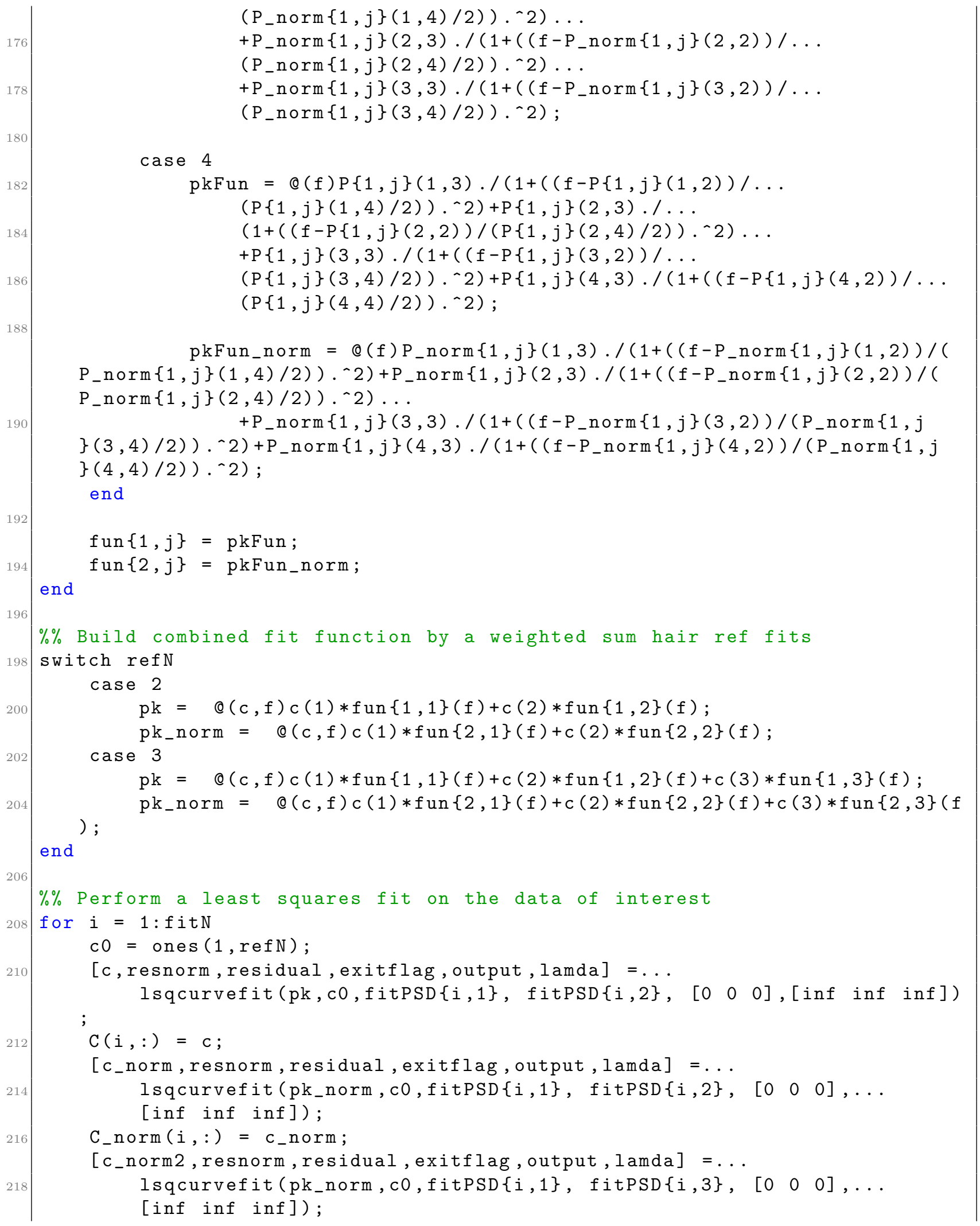


Rodrigo Sarlo App C: Hair Frequency Response Decomposition Top-Level Algorithm 107

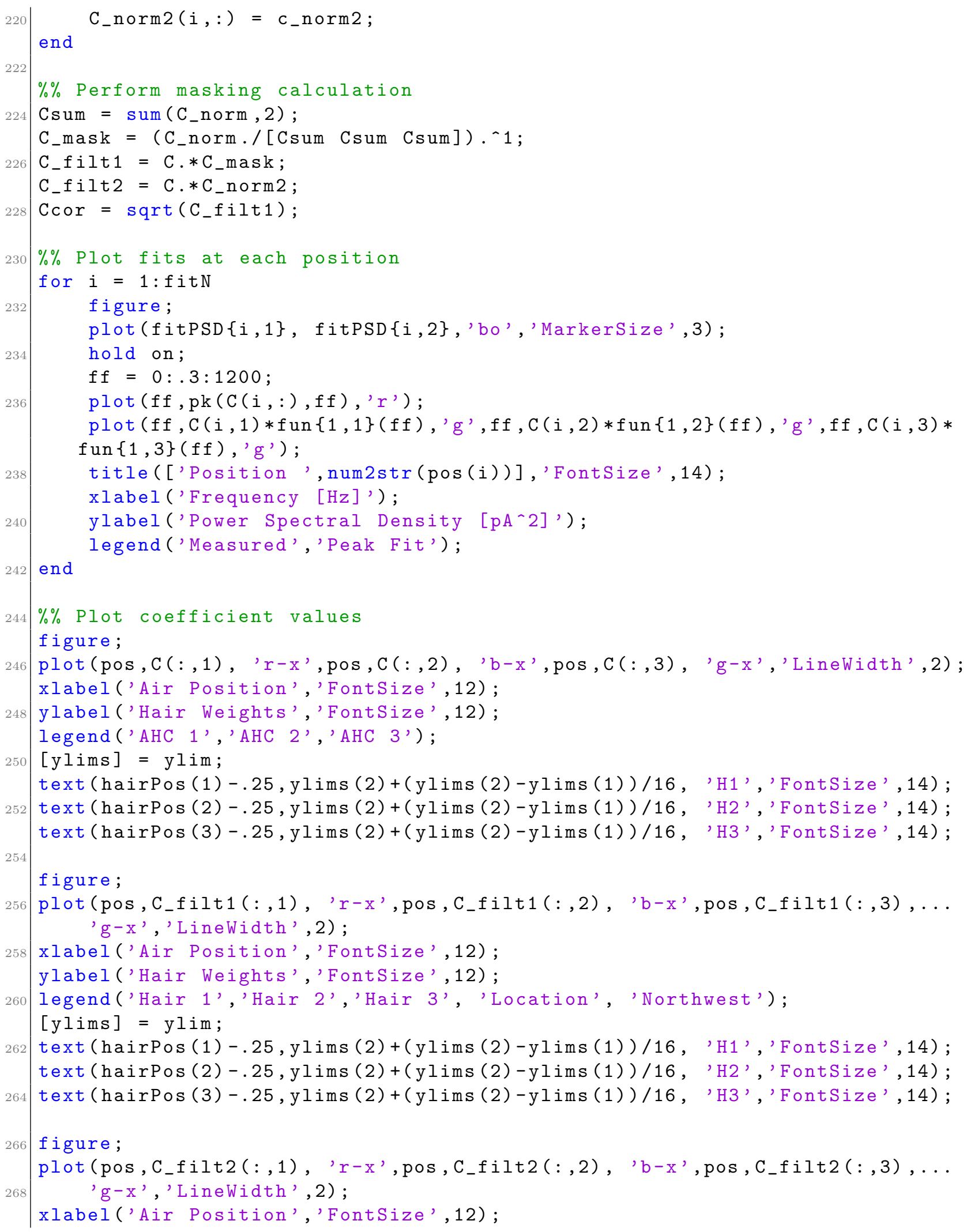


Rodrigo Sarlo App C: Hair Frequency Response Decomposition Top-Level Algorithm 108

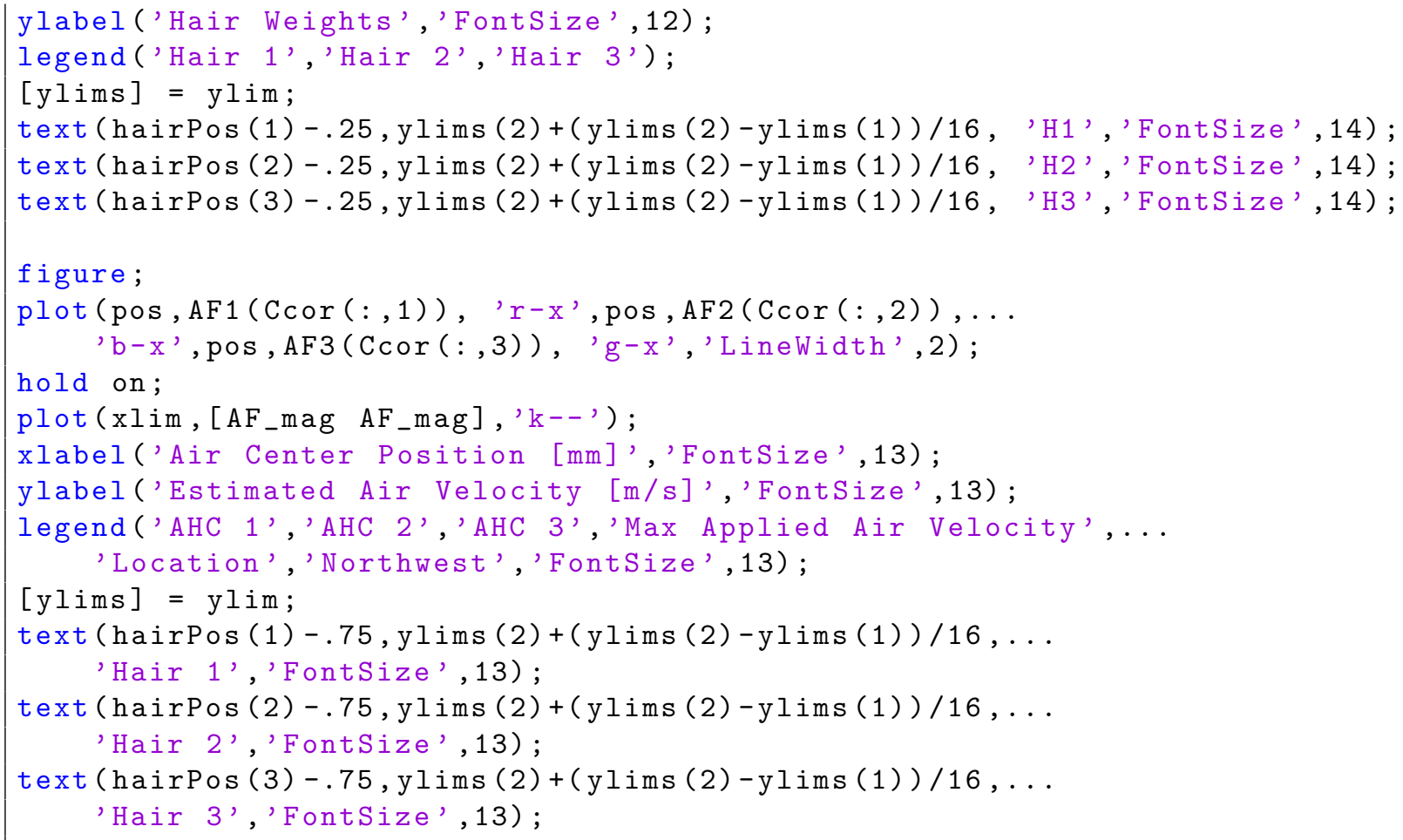

